\title{
THE HYPERBOLIC YANG-MILLS EQUATION FOR CONNECTIONS IN AN ARBITRARY TOPOLOGICAL CLASS
}

\author{
SUNG-JIN OH AND DANIEL TATARU
}

\begin{abstract}
This is the third part of a four-paper sequence, which establishes the Threshold Conjecture and the Soliton-Bubbling vs. Scattering Dichotomy for the energy critical hyperbolic Yang-Mills equation in the $(4+1)$-dimensional Minkowski space-time. This paper provides basic tools for considering the dynamics of the hyperbolic Yang-Mills equation in an arbitrary topological class at an optimal regularity.

We generalize the standard notion of a topological class of connections on $\mathbb{R}^{d}$, defined via a pullback to the one-point compactification $\mathbb{S}^{d}=\mathbb{R}^{d} \cup\{\infty\}$, to rough connections with curvature in the critical space $L^{\frac{d}{2}}\left(\mathbb{R}^{d}\right)$. Moreover, we provide excision and extension techniques for the Yang-Mills constraint (or Gauss) equation, which allow us to efficiently localize Yang-Mills initial data sets. Combined with the results in the previous paper [21, we obtain local well-posedness of the hyperbolic Yang-Mills equation on $\mathbb{R}^{1+d}(d \geq 4)$ in an arbitrary topological class at optimal regularity in the temporal gauge (where finite speed of propagation holds). In addition, in the energy subcritical case $d=3$, our techniques provide an alternative proof of the classical finite energy global well-posedness theorem of Klainerman-Machedon [11, while also removing the smallness assumption in the temporalgauge local well-posedness theorem of Tao [29].

Although this paper is a part of a larger sequence, the materials presented in this paper may be of independent and general interest. For this reason, we have organized the paper so that it may be read separately from the sequence.
\end{abstract}

\section{CONTEnts}

1. Introduction

1.1. Connections on a vector bundle with structure group $\mathbf{G}$

1.2. Global gauges and topological classes of $C^{\infty}$ connections

1.3. Global gauges for rough $\mathbf{G}$-bundles

1.4. Topological classes of rough connections

1.5. Hyperbolic Yang-Mills equation

1.6. Excision and extension of Yang-Mills initial data

1.7. Local theory in an arbitrary topological class

1.8. Topological classes, instantons and harmonic Yang-Mills connections on $\mathbb{R}^{4}$

2. Notation and conventions

3. Connections with $L^{\frac{d}{2}}$-curvature

3.1. G-valued functions at critical regularity

3.2. Patching procedures

3.3. Uhlenbeck lemmas and elliptic regularity $27 \mid$

3.4. Good global gauge theorem on the ball 30

3.5. Good global gauge theorem on the whole space 31

3.6. Topological classes of rough connections 
4. Excision, gluing and extension of Yang-Mills initial data sets 35

4.1. Solvability results for the inhomogeneous Gauss equation 35

4.2. Initial data surgery 38

5. The local theory for the hyperbolic Yang-Mills equation $\quad 40$

5.1. Gauge equivalent classes of connections $\quad 40$

5.2. Local theory at optimal regularity for dimensions $d \geq 4 \quad 41$

5.3. Local theory in dimension $d=3$

6. Harmonic Yang-Mills connections with compact structure group $\quad 47$

References $\quad 50$

\section{INTRODUCTION}

The subject of this paper is the $(d+1)$-dimensional hyperbolic Yang-Mills equation with compact noncommutative structure group. Our goal is two-fold:

- To describe, topologically and analytically, the Yang-Mills initial data sets at the optimal $L^{2}$-Sobolev regularity;

- To provide a good local theory for solutions at the optimal $L^{2}$-Sobolev regularity.

In each case, we consider two model base spaces: Either a ball $B_{R}=\left\{x \in \mathbb{R}^{d}:|x|<R\right\}$ or the whole space $\mathbb{R}^{d}$ for the first goal, and (suitable time restrictions of) their respective domains of dependence $\mathcal{D}\left(B_{R}\right)=\left\{(t, x) \in \mathbb{R}^{1+d}:|t|+|x|<R\right\}$ and $\mathcal{D}\left(\mathbb{R}^{d}\right)=\mathbb{R}^{1+d}$ for the second goal.

The main results of this paper may be classified into three classes:

(1) Good global gauge and topological class of rough connections. Motivated by the optimal regularity theory for the hyperbolic Yang-Mills equation, we consider locally-defined connections on a subset of $\mathbb{R}^{d}$ with $L^{\frac{d}{2}}$-curvature. Patching together the local gauges, we show that we can always produce good global gauges in the two model base spaces above (Theorems 1.4 and 1.5). Moreover, in whole space case, we use the asymptotics of the good global gauge potential to extend the notion of topological classes of connections to the rough setting. (Definition 1.8).

(2) Initial data surgery. We provide techniques for excising and extending Yang-Mills initial data sets, which are subject to the nonlinear Yang-Mills constraint (or Gauss) equation (Theorems 1.16 and 1.17). These are based on a sharp solvability result for the covariant divergence equation $\mathbf{D}^{\ell} e_{\ell}=h$ which preserves physical space support property (Theorem 1.14).

(3) Large data local theory. Using the ideas of initial data surgery and patching solutions, we show how to extend a small data well-posedness result in the temporal gauge to arbitrarily large data; the key is that causality (or finite speed of propagation) holds in the temporal gauge. Combined with the optimal regularity temporal gauge small data global well-posedness theorem proved in [21], we prove local well-posedness of the hyperbolic Yang-Mills equation in the temporal gauge for arbitrary critical Sobolev initial data in $d \geq 4$ (Theorem 1.22). In $d=3$, we obtain a generalization of a low regularity result of Tao [29], as well as an alternative proof of the classical result of Klainerman-Machedon [11]. 
In addition, in the last section we provide a review of the theory of harmonic YangMills equation on $\mathbb{R}^{4}$ using the topological framework developed in this paper. A particular emphasis is given to the recent sharp energy lower bound for non-instanton solutions due to Gursky-Kelleher-Streets [10], which clarifies the threshold energy for the energy critical hyperbolic Yang-Mills equation (and the Yang-Mills heat flow); namely, it is twice the ground state energy.

Remark 1.1. When restricted to the energy critical dimension $d=4$, the results in this paper constitute the third part of a four-paper sequence, whose principal aim is to prove the Threshold Theorem for the energy critical hyperbolic Yang-Mills equation. The four installments of the series are concerned with

(1) the caloric gauge for the hyperbolic Yang-Mills equation, [20].

(2) large data energy dispersed caloric gauge solutions, [21].

(3) topological classes of connections and large data local well-posedness, present article.

(4) soliton bubbling vs. scattering dichotomy for large data solutions, [22].

A short overview of the whole sequence is provided in the survey paper [23].

The present paper is mostly independent of the other papers in the series; the only exception is the small data well-posedness result for the hyperbolic Yang-Mills equation from [21] $(d \geq 4)$, which is used here as a black-box.

This paper is structured as follows. In the remainder of the introduction, we present the basic definitions and main results of this paper. For the notation and conventions that are not explained in the course of exposition, we refer the reader to Section 2. In Sections 36 , we elaborate and provide proofs of the results stated in the introduction.

1.1. Connections on a vector bundle with structure group G. Here we give a quick review of the basic theory of connections on vector bundles, and at the same time fix some notation and conventions. For a textbook treatment of these materials, we recommend [13, 14, 16].

Let $\mathbf{G}$ be a compact Lie group with Lie algebra $\mathfrak{g}$. We denote the adjoint action of $\mathbf{G}$ on $\mathfrak{g}$ by $\operatorname{Ad}(O) A=O A O^{-1}$, and the corresponding action of $\mathfrak{g}$ by $\operatorname{ad}(A) B=[A, B]$. We endow $\mathfrak{g}$ with an inner product $\langle\cdot, \cdot\rangle$ which is $A d$-invariant (or bi-invariant), i.e.,

$$
\langle A, B\rangle=\langle A d(O) A, A d(O) B\rangle \quad A, B \in \mathfrak{g}, O \in \mathbf{G} .
$$

Such an $A d$-invariant inner product always exists if $\mathbf{G}$ is compact. Indeed, from any inner product $\langle\cdot, \cdot\rangle^{\prime}$, we may construct an $A d$-invariant inner product by applying $A d(O)$ to each input and averaging in $O \in \mathbf{G}$.

The main objects we consider are connections $\mathbf{D}$ on a vector bundle on some smooth base manifold $X$ with structure group $\mathbf{G}$. Here we recall the standard local definition of a vector bundle in the smooth and continuous cases, which will be most useful later:

Definition 1.2. A $C^{\infty}$ [resp. $C^{0}$ ] vector bundle $\eta$ on a smooth manifold $X$ with fibers modeled on a vector space $V$ consists of the following objects:

- An open cover $\left\{U_{\alpha}\right\}$ of $X$;

- For each pair $U_{\alpha}, U_{\beta}$, a $C^{\infty}$ [resp. $C^{0}$ ] transition map $O_{(\alpha \beta)}: U_{\alpha} \cap U_{\beta} \rightarrow$ Aut $(V)$, which satisfy the following cocycle properties:

(1) $O_{(\alpha \alpha)}=I \quad$ on $U_{\alpha}\left(=U_{\alpha} \cap U_{\alpha}\right)$, 
(2) $O_{(\alpha \gamma)}=O_{(\alpha \beta)} O_{(\beta \gamma)} \quad$ on $U_{\alpha} \cap U_{\beta} \cap U_{\gamma}$.

Suppose that a Lie group $\mathbf{G}$ acts on $V$, in the sense that there exists a smooth representation $\rho: \mathbf{G} \rightarrow \operatorname{Aut}(V)$. We say that $\eta$ has structure group $\mathbf{G}$ if the transition functions may be lifted to $C^{\infty}\left[\right.$ resp. $\left.C^{0}\right] \mathbf{G}$-valued cocyles, i.e.,

$$
O_{(\alpha \beta)}=\rho \circ \tilde{O}_{(\alpha \beta)} \quad \text { for some } \tilde{O}_{(\alpha \beta)}: U_{\alpha} \cap U_{\beta} \rightarrow \mathbf{G}
$$

so that $\left\{\tilde{O}_{(\alpha \beta)}\right\}$ satisfy the cocycle property.

For simplicity, throughout the paper we omit the representation $\rho$ and denote the lifted cocycles $\tilde{O}_{(\alpha \beta)}$ by $O_{(\alpha \beta)}$.

In the local formulation, vector bundles with structure group $\mathbf{G}$ defined by the data sets $\left\{U_{\alpha}, O_{(\alpha \beta)}\right\}$ and $\left\{U_{\alpha^{\prime}}^{\prime}, O_{\left(\alpha^{\prime} \beta^{\prime}\right)}^{\prime}\right\}$ are isomorphic if and only if there exists a common refinement $\left\{V_{\gamma}\right\}$ of $\left\{U_{\alpha}\right\}$ and $\left\{U_{\alpha^{\prime}}^{\prime}\right\}$, so that $V_{\gamma} \subseteq U_{\alpha(\gamma)} \cap U_{\alpha^{\prime}(\gamma)}$ and $C^{\infty}\left[\right.$ resp. $\left.C^{0}\right]$ functions $P_{(\gamma)}: V_{\gamma} \rightarrow \mathbf{G}$ so that

$$
P_{(\gamma)} O_{(\alpha(\gamma) \alpha(\delta))}=O_{\left(\alpha^{\prime}(\gamma) \alpha^{\prime}(\delta)\right)}^{\prime} P_{(\delta)} \quad \text { on } V_{\gamma} \cap V_{\delta}
$$

By the topological or isomorphism class of a vector bundle $\eta$, we mean the class of all vector bundles isomorphic to $\eta$.

The open cover $\left\{U_{\alpha}\right\}$ in Definition 1.2 provides subsets on which $\eta$ is isomorphic to the trivial bundle $U_{\alpha} \times V$, and the transition maps $\left\{O_{(\alpha \beta)}\right\}$ describe how these local trivial bundles are patched together. We call an isomorphism $\eta \uparrow_{U_{\alpha}} \rightarrow U_{\alpha} \times V$ a local gauge (or local trivializations), and refer to $O_{(\alpha \beta)}$, viewed as an isomorphism between two trivial bundles $U_{\alpha} \times V$, as a local gauge transformation. Moreover, we use the term global gauge for a global isomorphism from $\eta \rightarrow X \times V$ (if it exists), and global gauge transformation for a $\mathbf{G}$-valued function on $X$, viewed as an isomorphism between such trivial bundles.

Let $\eta$ be a $C^{\infty}$ vector bundle with structure group $\mathbf{G}$, defined by the data $\left\{U_{\alpha}, O_{(\alpha \beta)}\right\}$. A section $s$ of $\eta$ consists of local data $s_{(\alpha)}$ (the local expression for $s$ in the local gauge on $U_{\alpha}$ ), which are smooth functions $s_{(\alpha)}: U_{\alpha} \rightarrow V$ satisfying the compatibility condition

$$
s_{(\alpha)}=O_{(\alpha \beta)} s_{(\beta)} \quad \text { on } U_{\alpha} \cap U_{\beta} \text {. }
$$

A connection $\mathbf{D}$ on $\eta$ consists of local data $\mathrm{d}+A_{(\alpha)}$, where each $A_{(\alpha)}$ is a smooth $\mathfrak{g}$-valued 1-form on $U_{\alpha}$ satisfying the compatibility condition:

$$
A_{(\alpha)}=A d\left(O_{(\alpha \beta)}\right) A_{(\beta)}-\partial O_{(\alpha \beta)} O_{(\alpha \beta)}^{-1} \quad \text { on } U_{\alpha} \cap U_{\beta} .
$$

We call $A_{(\alpha)}$ a gauge potential for $\mathbf{D}$ in the local gauge $U_{\alpha}$.

Observe that $\mathbf{D}$ defines a first order differential on the space of smooth sections of $\eta$, in the sense that $\mathbf{D}(f s)=\mathrm{d} f s+f \mathbf{D} s$ for any function $f$ and any section $s$. The space of all connections is denoted by $\mathcal{A}(\eta)$. As is well-known, $\mathcal{A}(\eta)$ has the structure of an affine space, in the sense that the difference of two connections $\mathbf{D}$ and $\mathbf{D}^{\prime}$ is a 1 -form taking values in the adjoint bundle $a d(\eta)$ (defined with the same data as $\eta$, but where $V=\mathfrak{g}$ and $O_{(\alpha \beta)}$ acts on $V$ on the left by the adjoint action).

The curvature 2 -form of $\mathbf{D}$ is defined by the relation

$$
F[\mathbf{D}](X, Y) \cdot s=\mathbf{D}_{X} \mathbf{D}_{Y} s-\mathbf{D}_{Y} \mathbf{D}_{X} s-\mathbf{D}_{[X, Y]} s
$$

Locally, it takes the form

$$
F_{(\alpha)}=\mathrm{d} A_{(\alpha)}+\frac{1}{2}\left[A_{(\alpha)} \wedge A_{(\alpha)}\right] \quad \text { on } U_{\alpha},
$$


and different local data are related to each other by

$$
F_{(\alpha)}=A d\left(O_{(\alpha \beta)}\right) F_{(\beta)} \quad \text { on } U_{\alpha} \cap U_{\beta} .
$$

In other words, $F$ is an $a d(\eta)$-valued 2-form on $X$.

Finally, we introduce the notion of the associated principal $\mathbf{G}$-bundle, which is the bundle with data the $\left\{U_{\alpha}, O_{(\alpha \beta)}\right\}$ and with the fibers modeled on the group $\mathbf{G}$, where the transition functions $O_{(\alpha \beta)}$ act on $\mathbf{G}$ by right multiplication. From the local viewpoint, it is simply a way to encapsulate the data $\left\{U_{\alpha}, O_{(\alpha \beta)}\right\}$ without reference to any vector space $V$. Principal bundles may serve as an alternative starting point for developing the theory of vector bundles (cf. Kobayashi-Nomizu [13, 14]).

1.2. Global gauges and topological classes of $C^{\infty}$ connections. In the following few subsections, we specialize to the cases $X=B_{R}$ (a ball of radius $R$ in $\mathbb{R}^{d}$ ) or $\mathbb{R}^{d}$. Eventually, we aim to give a suitable definition of connections at the optimal regularity, and introduce the notion of topological classes of such connections. Before we embark on these goals, we first review the simple case of a $C^{\infty}$ connection with a compactly supported curvature.

We start with the case $X=B_{R}$. Since $B_{R}$ is contractible, all $C^{\infty}$ vector bundles over $B_{R}$ are trivial; more precisely, a global gauge (or trivialization) of $\eta$ on $B_{R}$ can be constructed by parallel transportation with respect to $\mathbf{D}$ along each ray starting from the center $x_{0}$ of $B_{R}$. We obtain a representative $A$ of $\mathbf{D}$ on $B_{R}$ such that

$$
A \in C^{\infty}\left(B_{R} ; \mathfrak{g}\right) \text {. }
$$

Moreover, $\left(x-x_{0}\right)^{j} A_{j}=0$ by the parallel transport condition.

Next, we consider the case $X=\mathbb{R}^{d}$. Since $\mathbb{R}^{d}$ is contractible, too, all $C^{\infty}$ vector bundles over $\mathbb{R}^{d}$ are trivial. However, when the vector bundles is endowed with a compactly supported curvature, we may define their topological class by viewing them as bundles on the compactification $\mathbb{R}^{d} \cup\{\infty\}$, which is homeomorphic to $\mathbb{S}^{d}=\left\{X \in \mathbb{R}^{d+1}:|X|=1\right\}$. More precisely, consider the stereographic projection

$$
\Sigma: \mathbb{S}^{d} \rightarrow \mathbb{R}^{d}, \quad\left(X^{1}, \ldots, X^{d+1}\right) \mapsto\left(\frac{X^{1}}{1-X^{d+1}}, \ldots, \frac{X^{d}}{1-X^{d+1}}\right) .
$$

Note that the pullback of $(\eta, \mathbf{D})$ along $\boldsymbol{\Sigma}$, which we denote by $\left(\boldsymbol{\Sigma}^{*} \eta, \boldsymbol{\Sigma}^{*} \mathbf{D}\right)$, obeys $F\left[\boldsymbol{\Sigma}^{*} \mathbf{D}\right]=0$ on $U_{\infty}^{\prime}=\left\{X \in \mathbb{S}^{d}: 0<X^{d+1}<1\right\}=\Sigma^{-1}\left(\mathbb{R}^{d} \backslash B_{1}\right)$. Since $U_{\infty}^{\prime}$ is simply connected, the pullback bundle $\Sigma^{*} \eta$ is isomorphic to the trivial bundle $U_{\infty}^{\prime} \times V$ [13, Corollary 9.2], which may be easily extended to $U_{\infty}=\left\{X \in \mathbb{S}^{d}: X^{d+1}>0\right\}$. Therefore, $\Sigma^{*} \eta$ extends to a smooth vector bundle on $\mathbb{S}^{d}$. The topological class of $(\eta, \mathbf{D})$ may be defined to be that of the extended bundle on $\mathbb{S}^{d}$.

Since $\mathbb{S}^{d}$ is covered by with two contractible open sets, namely $U_{0}=\mathbb{S}^{d} \backslash\{(0, \ldots, 0,1)\}$ and $U_{\infty}=\mathbb{S}^{d} \backslash\{(0, \ldots, 0,-1)\}$, the topological class of the bundle on $\mathbb{S}^{d}$ is determined by the transition map in-between. At the level of $\eta$, it is the transition map $O$ between $\mathbb{R}^{d}$, on which there exists a local representative $\mathbf{D}=\mathrm{d}+A$ with $A(0)=0$ and $x^{j} A_{j}=0$ (parallel transport along radial rays from 0 ), and $\mathbb{R}^{d} \backslash B_{1}$, on which $\mathbf{D}=\mathrm{d}$. On $\mathbb{R}^{d} \backslash B_{1}$, we have

$$
A=-\partial_{x} O O^{-1} \text {. }
$$

Moreover, since $x^{j} A_{j}=0$, it follows that $x^{j} \partial_{j} O=0$ on $\mathbb{R}^{d} \backslash B_{1}$, i.e., $O(x)=O\left(\frac{x}{|x|}\right)$ for $|x| \geq 1$. Defining $O_{(\infty)}: \mathbb{R}^{d} \backslash\{0\} \rightarrow \mathbf{G}, O_{(\infty)}(x)=O\left(\frac{x}{|x|}\right)$ and introducing a smooth function $\chi$ such that $1-\chi$ is compactly supported, we arrive at: 
Theorem 1.3. Let $\mathbf{D}$ be a $C^{\infty}$ connection on a $C^{\infty}$ vector bundle $\eta$ on $\mathbb{R}^{d}$, whose curvature is compactly supported. Then there exists a global gauge for $\eta$ in which the global gauge potential $A=\mathbf{D}-\mathrm{d}$ admits a decomposition of the form

$$
A=-\chi O_{(\infty) ; x}+B
$$

where $O_{(\infty)}(x)$ is a smooth 0 -homogeneous map into $\mathbf{G}$ and $B \in C_{c}^{\infty}\left(\mathbb{R}^{d} ; \mathfrak{g}\right)$.

It is not difficult to see that $O_{(\infty)}$, which we call a gauge at infinity for $A$, is defined uniquely up to homotopy (cf. Proposition [1.6). The homotopy class $\left[O_{(\infty)}\right]$, which is defined intrinsically without reference to the pullback procedure, determines the topological class 1 of the extended pullback bundle on $\mathbb{S}^{d}$. Hence, any topological invariants of the extended pullback bundle depend only on $\left[O_{(\infty)}\right]$.

Characteristic classes are important invariants of a vector (or principal) G-bundle. On $\mathbb{S}^{d}$, by the Chern-Weil theory [14, Chapter XII], these may be defined in terms of a connection $\mathrm{D}$ as follows. Given any symmetric $A d$-invariant $k$-linear function $f$ on $\mathfrak{g}$, we call the $2 k$-form

$$
f(F[\mathbf{D}], \ldots, F[\mathbf{D}])=f\left(F_{j_{1} j_{2}}, \ldots, F_{j_{d-1} j_{d}}\right) \mathrm{d} x^{j_{1}} \wedge \mathrm{d} x^{j_{2}} \wedge \cdots \wedge \mathrm{d} x^{j_{d}}
$$

the characteristic class associated to $f$. This $2 k$-form is closed and is invariant, up to an exact form, in the choice of a connection $\mathbf{D}$ on the bundle; hence it defines a cohomology class in $H^{2 k}\left(\mathbb{S}^{d}\right)$, which depends only on the isomorphism class of the bundle. Moreover, when $d=2 k$, the integral

$$
\chi_{f}=\int_{\mathbb{S}^{d}} f(F[\mathbf{D}], \ldots, F[\mathbf{D}])
$$

called the characteristic number, is also an invariant of the bundle.

Now, as an application of Theorem 1.3 , consider a $C^{\infty}$ connection $\mathbf{D}$ on $\mathbb{R}^{d}$ with compactly supported curvature. Then $\chi_{f}$ of the pullback bundle equals

$$
\chi_{f}=\int_{\mathbb{R}^{d}} f(F[\mathbf{D}], \ldots, F[\mathbf{D}]),
$$

and depends only on $\left[O_{(\infty)}\right]$ in Theorem 1.3 .

An important special case of the above theory is when $d=4$ and $\mathbf{G}=S U(2)$, and we take $f(A, B)=\frac{1}{8 \pi^{2}} \operatorname{tr}(A B)$. The corresponding characteristic number, given by the integral formula

$$
c_{2}=\frac{1}{8 \pi^{2}} \int_{\mathbb{R}^{4}} \operatorname{tr}(F \wedge F),
$$

is called the second Chern number. It is always an integer, and it classifies the topological classes of $S U(2)$-bundles. For more on characteristic classes, we refer the reader to [16].

1.3. Global gauges for rough G-bundles. We are now ready to describe our first set of results. Motivated by the desire to study the hyperbolic Yang-Mills equation (cf. Section 1.5) at the optimal scaling-invariant regularity, our aim here is to sharpen (1.1) and (1.3) in two ways:

(1) To obtain quantitative bounds for $A$ in a "good global gauge" in terms of $F$;

(2) To relax the condition for $F$ to the scaling-invariant condition $F \in L^{\frac{d}{2}}(X)$.

\footnotetext{
${ }^{1}$ Strictly speaking, $O_{(\infty)}$ in Theorem 1.3 directly determines only the smooth isomorphism class, which in turn determines the topological (i.e., $C^{0}$ ) isomorphism class by a density argument.
} 
In what follows, we restrict to $d \geq 3$ (which, for instance, avoids the case $L^{\frac{d}{2}}=L^{1}$ ).

To set up the scene, we start with the definition of connections with $L_{l o c}^{\frac{d}{2}}$ curvature. Let $X$ be an open subset of $\mathbb{R}^{d}$. For $k \in \mathbb{R}$ and $p \in[1, \infty]$, we introduce

$$
\mathcal{G}_{l o c}^{k, p}(X)=\left\{O \in W_{l o c}^{k, p}\left(X ; \mathbb{R}^{N \times N}\right): O(x) \in \mathbf{G} \text { for a.e. } x \in X\right\} .
$$

The relevant regularity class is $\mathcal{G}_{l o c}^{2, \frac{d}{2}}$, which turns to be closed under multiplication and inverse (see Lemmas 3.1, 3.3 and 3.4 below). In parallel to Section 1.1, we define a $\mathcal{G}_{\text {loc }}^{2, \frac{d}{2}}$ (principal) G-bundle on $X \subseteq \mathbb{R}^{d}$ by the data:

- An open cover $\left\{U_{\alpha}\right\}$ of $X$;

- A transition function $O_{(\alpha \beta)} \in \mathcal{G}_{l o c}^{2, \frac{d}{2}}\left(U_{\alpha} \cap U_{\beta}\right)$ for every $\alpha, \beta$, obeying the cocycle conditions:

(1) $O_{(\alpha \alpha)}=i d$ on each $U_{\alpha}$;

(2) $O_{(\alpha \beta)} \cdot O_{(\beta \gamma)}=O_{(\alpha \gamma)}$ on each $U_{\alpha} \cap U_{\beta} \cap U_{\gamma}$.

An open cover $\left\{V_{\gamma}\right\}$ is a refinement of $\left\{U_{\alpha}\right\}$ if there exists a function $\alpha=\alpha(\gamma)$ such that $V_{\gamma} \subseteq U_{\alpha(\gamma)}$. We say that two data sets $\left\{U_{\alpha}, O_{(\alpha \beta)}\right\}$ and $\left\{U_{\alpha^{\prime}}^{\prime}, O_{\left(\alpha^{\prime} \beta^{\prime}\right)}^{\prime}\right\}$ define an equivalent $\mathcal{G}_{\text {loc }}^{2, \frac{d}{2}}$ bundle if there exists a common refinement $V_{\gamma}$ of the open covers and $P_{(\gamma)} \in \mathcal{G}_{l o c}^{2, \frac{d}{2}}\left(V_{\gamma}\right)$ such that

$$
P_{(\delta)} \cdot O_{(\alpha(\delta) \alpha(\gamma))}=O_{\left(\alpha^{\prime}(\delta) \alpha^{\prime}(\gamma)\right)}^{\prime} \cdot P_{(\gamma)} \quad \text { on } V_{\gamma} \cap V_{\delta}
$$

A $W_{l o c}^{1, \frac{d}{2}}$ connection $\mathbf{D}$ on the bundle defined by $\left\{U_{\alpha}, O_{(\alpha \beta)}\right\}$ is given by the local data:

- A 1-form $A_{(\alpha)} \in W_{l o c}^{1, \frac{d}{2}}\left(U_{\alpha} ; \mathfrak{g}\right)$ for each $\alpha$, called the local representative of $\mathbf{D}$ on $U_{\alpha}$, satisfying the compatibility condition

$$
A_{(\alpha)}=\operatorname{Ad}\left(O_{(\alpha \beta)}\right) A_{(\beta)}-O_{(\alpha \beta) ; x} \quad \text { on each } U_{\alpha} \cap U_{\beta} .
$$

Given a $W_{l o c}^{1, \frac{d}{2}}$ connection $\mathbf{D}$, we define its curvature 2-form $F=F[\mathbf{D}]$ by the local data:

$$
F_{(\alpha)}=\mathrm{d} A_{(\alpha)}+\frac{1}{2}\left[A_{(\alpha)} \wedge A_{(\alpha)}\right] \quad \text { on each } U_{\alpha}
$$

We denote by $\mathcal{A}_{\text {loc }}^{1, \frac{d}{2}}(X)$ the space of all $W_{\text {loc }}^{1, \frac{d}{2}}$ connections on all $\mathcal{G}_{\text {loc }}^{2, \frac{d}{2}}$ bundles on $X$. By the compatibility property of $F_{(\alpha)}$ (algebraically the same as in the smooth case), note that

$$
|F|=\left|F_{(\alpha)}\right|=\sqrt{\left\langle F_{(\alpha)}, F_{(\alpha)}\right\rangle} \quad \text { on each } U_{\alpha}
$$

is a well-defined element of $L_{l o c}^{\frac{d}{2}}(X)$.

Consider the case $X=B_{R}$. In order to state quantitative bounds for the gauge potential in a "good gauge", we introduce the inner $\left(L^{\frac{d}{2}}-\right)$ concentration scale with threshold $\epsilon_{*}$ of a connection $\mathbf{D}$, defined as follows:

$$
\underline{r}_{c}^{\epsilon_{*}}[\mathbf{D}]=\sup \left\{r>0:\|F[\mathbf{D}]\|_{L^{\frac{d}{2}}\left(B_{r}(x) \cap X\right)} \leq \epsilon_{*} \text { for all } x \in X\right\} .
$$

Theorem 1.4 (Good gauge on a ball). Let $\mathbf{D} \in \mathcal{A}_{\text {loc }}^{1, \frac{d}{2}}\left(B_{R}\right)$ satisfy $F[\mathbf{D}] \in L^{\frac{d}{2}}\left(B_{R}\right)$ and $\underline{r}_{c}^{\epsilon *}[\mathbf{D}] \geq r$, for some $r>0$ and a sufficiently small $\epsilon_{*}>0$. Then there exists a global gauge 
in which the gauge potential $A$ for $\mathbf{D}$ satisfies

$$
\|A\|_{\dot{W}^{1, \frac{d}{2}}\left(B_{R}\right)} \lesssim_{\epsilon_{*}, \frac{R}{r}} 1
$$

If, in addition, $\mathbf{D}^{(n)} F \in L^{p}\left(B_{R}\right)$ for some nonnegative integer $n$ and $p \in(1, \infty)$ such that $p \geq \frac{d}{n+2}$, then $A \in W^{n+1, p}\left(B_{R}\right)$.

Theorem 1.4 tells us that given any connection on a ball with $L^{\frac{d}{2}}$-curvature, there exists a good gauge in which the a-priori bound (1.6) holds. When $\|F[\mathbf{D}]\|_{L^{\frac{d}{2}\left(B_{R}\right)}}$ is sufficiently small (with the threshold depending on $d$ ), Theorem 1.4 is the classical result of Uhlenbeck [30]. The general case is proved by appropriately patching up local applications of Uhlenbeck's lemma.

Next, we consider the case $X=\mathbb{R}^{d}$. To proceed, we need an additional concept. We define the outer $\left(L^{\frac{d}{2}}-\right)$ concentration radius with threshold $\epsilon_{*}$ of a connection $\mathbf{D}$ to be

$$
\underline{R}_{c}^{\epsilon_{*}}[\mathbf{D}]=\inf \left\{r>0:\|F[\mathbf{D}]\|_{L^{\frac{d}{2}}\left(\mathbb{R}^{d} \backslash B_{r}(x)\right)} \leq \epsilon_{*} \text { for some } x \in \mathbb{R}^{d}\right\} .
$$

Let $1-\chi \in C_{c}^{\infty}\left(\mathbb{R}^{d}\right)$ be fixed.

Theorem 1.5 (Good global gauge on $\left.\mathbb{R}^{d}\right)$. Let $\mathbf{D} \in \mathcal{A}_{\text {loc }}^{1, \frac{d}{2}}\left(\mathbb{R}^{d}\right)$ satisfy $F[\mathbf{D}] \in L^{\frac{d}{2}}\left(\mathbb{R}^{d}\right)$, as well as $\underline{r}_{c}^{\epsilon_{*}}[\mathbf{D}] \geq r$ and $\underline{R}_{c}^{\epsilon_{*}}[\mathbf{D}] \leq R$ for some $0<r \leq R$ and a universal small constant $\epsilon_{*}>0$. Then there exists exists a global gauge on $\mathbb{R}^{d}$, in which the gauge potential $A \in \dot{W}_{\text {loc }}^{1, \frac{d}{2}}\left(\mathbb{R}^{d}\right)$ for $\mathbf{D}$ admits a decomposition of the form

$$
A=-\chi(\cdot / R) O_{(\infty) ; x}+B
$$

where $O_{(\infty)}(x)$ is a smooth 0 -homogeneous map into $\mathbf{G}$ and $B \in \dot{W}^{1, \frac{d}{2}}\left(\mathbb{R}^{d} ; \mathfrak{g}\right)$. Moreover,

$$
\|B\|_{\dot{W}^{1, \frac{d}{2}}} \lesssim_{\epsilon_{*}, \frac{R}{r}} 1, \quad\left\|O_{(\infty)}\right\|_{C^{N}\left(\mathbb{S}^{d-1}\right)} \lesssim_{\epsilon_{*}, \frac{R}{r}, N} 1 \quad \text { for all } N \geq 0 .
$$

If, in addition, $\mathbf{D}^{(n)} F \in L^{p}\left(B_{R}\right)$ for some nonnegative integer $n$ and $p \in(1, \infty)$ such that $p \geq \frac{d}{n+2}$, then $B \in \dot{W}^{n+1, p}\left(\mathbb{R}^{d}\right)$.

Thanks to Theorems 1.4 and 1.5, we may identify any connection $\mathbf{D} \in \mathcal{A}^{1, \frac{d}{2}}(X)$ with a gauge potential $A \in W_{l o c}^{1, \frac{d}{2}}(X)$ in a good global gauge. In the rest of the introduction, we adopt the convention of referring to a connection $\mathbf{D}$ on $B_{R}$ or $\mathbb{R}^{d}$ by its global gauge potential A.

1.4. Topological classes of rough connections. Given a $W_{l o c}^{1, \frac{d}{2}}$ connection $A$ on $\mathbb{R}^{d}$, we call a pair $\left(O_{(\infty)}, B\right)$ of a smooth 0-homogeneous map into $\mathbf{G}$ and an element in $\dot{W}^{1, \frac{d}{2}}\left(\mathbb{R}^{d} ; \mathfrak{g}\right)$ a good representative of $A$ if $A=-\chi O_{(\infty) ; x}+B$ for some $1-\chi \in C_{c}^{\infty}\left(\mathbb{R}^{d}\right)$. We furthermore call $O_{(\infty)}$ a gauge (transformation) at infinity for $A$. Theorem 1.5 insures that a good representative always exists provided that $F[A] \in L^{\frac{d}{2}}$.

Recall that when the curvature is smooth and compactly supported, the topological class of $A$ is classified by the homotopy class of its gauge at infinity $O_{(\infty)}$. We extend the definition of the topological class to a rough connections on $\mathbb{R}^{d}$ with $L^{\frac{d}{2}}$-curvature using this classification. We need the following preliminary results: 
Proposition 1.6. Let $A \in \mathcal{A}_{\text {loc }}^{1, \frac{d}{2}}\left(\mathbb{R}^{d}\right)$ satisfy $F[A] \in L^{\frac{d}{2}}\left(\mathbb{R}^{d}\right)$, and let $\left(O_{(\infty)}, B\right)$ be a good representative of $A$.

(1) If $\left(O_{(\infty)}^{\prime}, B^{\prime}\right)$ is another good representation of $A$, then $O_{(\infty)}$ is homotopic to $O_{(\infty)}^{\prime}$.

(2) Conversely, given any smooth $O_{(\infty)}^{\prime}: \mathbb{S}^{d-1} \rightarrow \mathrm{G}$ homotopic to $O_{(\infty)}$, there exists another good representation $\left(O_{(\infty)}^{\prime}, B^{\prime}\right)$ of $A$.

Remark 1.7. For completeness, we make the trivial observation that the homotopy class of $O_{(\infty)}$ is independent of the choice of $\chi$, too.

Theorem 1.5, Proposition 1.6 and Remark 1.7 lead to the following:

Definition 1.8. Given an $L^{\frac{d}{2}}$-curvature connection $A$, we define the topological class $[A]$ of $A$ to be the homotopy class of $O_{(\infty)}: \mathbb{S}^{d-1} \rightarrow \mathbf{G}$ of a good representative (i.e., a gauge at infinity for $A$ ). If the topological class of $A^{\prime}$ is $[A]$, then we write $A^{\prime} \in[A]$.

Observe that the addition of a 1 -form $B$ in $\dot{W}^{1, \frac{d}{2}}\left(\mathbb{R}^{d} ; \mathfrak{g}\right)$ does not change the topological class of $A$, i.e.,

$$
A+B \in[A]
$$

In particular, by mollifying and cutting off $B$, we can easily find approximations by smooth connections with compactly supported curvature in the same topological class with respect to the distance $d_{\dot{W}^{1, \frac{d}{2}}}\left(A, A^{\prime}\right)=\left\|A-A^{\prime}\right\|_{\dot{W}^{1, \frac{d}{2}}\left(\mathbb{R}^{d} ; \mathfrak{g}\right)}$. Moreover, good representations of two connections with the same $O_{(\infty)}$ are path-connected with respect to the $d_{\dot{W}^{1, \frac{d}{2}}}$. By Proposition 1.6, it follows that each topological class is path-connected with respect to $d_{\dot{W}^{1, \frac{d}{2}}}$ up to global gauge transformations in $\mathcal{G}_{l o c}^{2, \frac{d}{2}}\left(\mathbb{R}^{d}\right)$.

Observe also that topological class is determined by the part of the connection where the $L^{\frac{d}{2}}$ norm of $F$ is concentrated. More precisely, we have:

Proposition 1.9. Let $A, A^{\prime} \in \mathcal{A}_{\text {loc }}^{1, \frac{d}{2}}\left(\mathbb{R}^{d}\right)$ satisfy $F[A], F\left[A^{\prime}\right] \in L^{\frac{d}{2}}\left(\mathbb{R}^{d}\right)$. Assume moreover that $A$ and $A^{\prime}$ are close in $L^{d}\left(B_{5 R}\right)$, and have small $L^{\frac{d}{2}}$ curvature outside $B_{R}$, i.e.,

$$
\left\|A-A^{\prime}\right\|_{L^{d}\left(B_{5 R}\right)} \leq \epsilon_{*}, \quad\|F[A]\|_{L^{\frac{d}{2}\left(\mathbb{R}^{d} \backslash B_{R}\right)}} \leq \epsilon_{*}, \quad\left\|F\left[A^{\prime}\right]\right\|_{L^{\frac{d}{2}}\left(\mathbb{R}^{d} \backslash B_{R}\right)} \leq \epsilon_{*},
$$

where $\epsilon_{*}>0$ is sufficiently small universal constant. Then $[A]=\left[A^{\prime}\right]$.

We now discuss some simple consequences of the above results. Given an $L^{\frac{d}{2}}$-curvature connection $A$, let $A^{n}$ be an approximation of $A$ in $d_{\dot{W}^{1,} \frac{d}{2}}$, such that each $A^{n}$ is smooth and $F\left[A^{n}\right]$ is compactly supported. For any symmetric $A d$-invariant $k$-linear function $f$ on $\mathfrak{g}$, the associated characteristic classes of the pullback bundles $\left(\Sigma^{*} \eta, \Sigma^{*} A^{n}\right)$ are independent of $n$ (for sufficiently large $n$ ), as well as of the approximating sequence. Moreover, when $d=2 k$, the characteristic numbers obey

$$
\chi_{f}=\int_{\mathbb{R}^{d}} f\left(F\left[A^{n}\right], \ldots, F\left[A^{n}\right]\right) \rightarrow \int_{\mathbb{R}^{d}} f(F[A], \ldots, F[A])
$$

by continuity of the integral with respect to $\left\|A-A^{\prime}\right\|_{\dot{W}^{1, \frac{d}{2}}\left(\mathbb{R}^{d} ; \mathfrak{g}\right)}$. Hence we recover the following result of Uhlenbeck [31]:

Corollary 1.10. The characteristic numbers $\chi_{f}$, defined as in (1.4), depend only on $[A]$. In particular, they vanish for [0]. 
As another corollary of Theorem 1.5, we obtain a characterization of the topologically trivial class (i.e., the topological class of the trivial connection $A=0$ ):

Corollary 1.11. The space of topologically trivial connections with finite $L^{\frac{d}{2}}$ curvature correspond exactly to

$$
\mathcal{A}_{0}^{1, \frac{d}{2}}\left(\mathbb{R}^{d}\right)=\left\{\mathbf{D}=\mathrm{d}+A: A \in \dot{W}^{1, \frac{d}{2}}\left(\mathbb{R}^{d} ; \mathfrak{g}\right)\right\} .
$$

All characteristic numbers associated to a connection $A$ in $\mathcal{A}_{0}^{1, \frac{d}{2}}\left(\mathbb{R}^{d}\right)$ vanish.

Remark 1.12. The preceding corollary implies that given any connection $A$ in the topologically trivial class, there exists a global representative $\tilde{A}$ in the space $\dot{W}^{1, \frac{d}{2}}\left(\mathbb{R}^{d} ; \mathfrak{g}\right)$. Note, however, that no quantitative bound on $\|\tilde{A}\|_{\dot{W}^{1, \frac{d}{2}}}$ is claimed; such a bound would rely on quantitative bounds on a homotopy of $O_{(\infty)}$ to the identity in terms of scaling-invariant bounds on $O_{(\infty)}$.

1.5. Hyperbolic Yang-Mills equation. The remainder of the introduction concerns the hyperbolic Yang-Mills equation. The purpose of this subsection is to provide a brief introduction to this equation.

Let $\mathbb{R}^{1+d}$ denote the $(d+1)$-dimensional Minkowski space, which is equipped with the Minkowski metric $\mathbf{m}_{\mu \nu}=\operatorname{diag}(-1,+1, \ldots,+1)$ in the rectangular coordinates $\left(x^{0}, x^{1}, \ldots, x^{d}\right)$. We will often write $t=x^{0}$, to emphasize the role of $x^{0}$ as (a choice of) a time function. Throughout this paper, we will use the usual convention of raising and lowering indices using the Minkowski metric, as well as summing up repeated upper and lower indices.

Consider a connection $\mathbf{D}$ on a vector bundle on $\mathbb{R}^{1+d}$ with structure group $\mathbf{G}$. By topological triviality of $\mathbb{R}^{d}$ (or Theorem [1.5] at low regularity), $\mathbf{D}$ at each $t$ may be identified with a global gauge potential $A$. The hyperbolic Yang-Mills equation on $\mathbb{R}^{1+d}$ for $A$ is the Euler-Lagrange equation associated with the formal Lagrangian action functional

$$
\mathcal{L}(A)=\frac{1}{2} \int_{\mathbb{R}^{1+d}}\left\langle F_{\alpha \beta}, F^{\alpha \beta}\right\rangle \mathrm{d} x \mathrm{~d} t,
$$

which takes the form

$$
\mathrm{D}^{\alpha} F_{\alpha \beta}=0 .
$$

Clearly, (1.9) is invariant under (smooth) gauge transformations. This equation possesses a conserved energy, given by

$$
\mathcal{E}_{\{t\} \times \mathbb{R}^{d}}(A)=\int_{\{t\} \times \mathbb{R}^{d}} \sum_{\alpha<\beta}\left|F_{\alpha \beta}\right|^{2} \mathrm{~d} x .
$$

Furthermore, (1.9) is invariant under the scaling

$$
A(t, x) \mapsto \lambda A(\lambda t, \lambda x) \quad(\lambda>0) .
$$

The scaling-invariant $L^{2}$-Sobolev norm is $\|A(t, \cdot)\|_{\dot{H} \frac{d-2}{2}}$. In particular, (1.9) is energy critical when $d=4$, in the sense that the conserved energy (which scales like $\|A(t, \cdot)\|_{\dot{H}^{1}}$ ) is invariant under the scaling.

We are interested in the initial value problem for (1.9) at the scaling-invariant $L^{2}$-Sobolev regularity. For this purpose we first formulate a gauge-covariant notion of initial data sets. 
We say that a pair $(a, e)$ of a gauge potential $a$ and a $\mathfrak{g}$-valued 1 -form $e$ on $\mathbb{R}^{d}$ is an initial data set for a solution $A$ to (1.9) if

$$
\left(A_{j}, F_{0 j}\right) \Upsilon_{\{t=0\}}=\left(a_{j}, e_{j}\right) .
$$

Here and throughout this paper, the roman letters stand for the spatial coordinates $x^{1}, \ldots, x^{d}$. Note that (1.9) with $\beta=0$ imposes the condition that

$$
\mathbf{D}^{j} e_{j}=\partial^{j} e_{j}+\left[a^{j}, e_{j}\right]=0 .
$$

This equation is the Gauss (or the constraint) equation for (1.9).

It turns out that (1.10) characterizes precisely those pairs $(a, e)$ which can arise as an initial data set. Thus we make the following definition:

Definition 1.13. An $\mathcal{H}^{\sigma}(\mathcal{O})$ (resp. $\dot{\mathcal{H}}^{\sigma}(\mathcal{O})$ or $\mathcal{H}_{\text {loc }}^{\sigma}(\mathcal{O})$ ) initial data set for the Yang-Mills equation is a pair $(a, e) \in H^{\sigma} \times H^{\sigma-1}(\mathcal{O})\left(\operatorname{resp} . \dot{H}^{\sigma} \times \dot{H}^{\sigma-1}(\mathcal{O})\right.$ or $\left.H_{l o c}^{\sigma} \times H_{l o c}^{\sigma-1}(\mathcal{O})\right)$ that satisfies the constraint equation (1.10).

Due to invariance under gauge transformations, (1.9) is not even formally well-posed when viewed as a PDE for $A$. In order to analyze (1.9) at the level of $A$, this invariance must be removed by fixing a representative (or a gauge). A simple and useful way is to require that

$$
A_{0}=0 \text {. }
$$

The gauge thus chosen is called temporal. In this gauge, (1.9) becomes a coupled system of wave and transport equations for the curl and divergence of $A$, respectively, and local well-posedness for regular data is easily follows. Moreover, in the regular case it is also easy to verify the finite speed of propagation property, in the sense that $A$ vanishes on the domain of dependence of the zero-set of the data.

The aforementioned coupled wave-transport system in the temporal gauge becomes difficult to analyze in the low regularity setting. Nonetheless, in [21, global well-posedness of (1.9) under (1.11) was proved for small data at the optimal $L^{2}$-Sobolev regularity (for dimensions $d \geq 4$ ), by first working in a gauge with more favorable structure (caloric gauge), and then estimating the gauge transformation to the temporal gauge.

At this point, one may imagine upgrading the small data result to large data local wellposedness by the following procedure:

(1) Constructing local-in-spacetime solutions from the small data result applied to suitable localizations of the initial data;

(2) Patch the local-in-spacetime solutions together by finite speed of propagation.

Though this strategy eventually works (see Section 1.7 below), this is not trivial. The primary reason is because the Gauss equation (1.10) is nonlocal, and thus initial data sets cannot be freely cut off. The next subsection is devoted to resolving this issue.

1.6. Excision and extension of Yang-Mills initial data. In this subsection we present the second set of results of this paper, which eventually lead to a useful excision-and-extension technique for Yang-Mills initial data. The first and main result is solvability of the inhomogeneous Gauss equation

$$
\left(\mathbf{D}^{(a)}\right)^{\ell} e_{\ell}=h
$$

while keeping good physical space support properties. 
Theorem 1.14. Let $d \geq 4$ and $a \in \dot{H}^{\frac{d-2}{2}}\left(\mathbb{R}^{d}\right)$. Given any convex open set $K$, there exists a solution operator $T_{a}$ for (1.12) satisfying the following conditions:

(1) (Boundedness) We have

$$
\left\|T_{a}[h]\right\|_{\dot{H} \frac{d-4}{2}} \lesssim\|a\|_{\dot{H} \frac{d-2}{2}}, L(K)\|h\|_{\dot{H} \frac{d-6}{2}},
$$

where $L(K)$ is a scaling-invariant quantity (i.e., $L(\lambda K)$ is independent of $\lambda>0$ ) defined in (4.2).

(2) (Exterior support property) If $h$ is supported outside the set

$$
\lambda K=\left\{\lambda\left(x-x_{K}\right) \in \mathbb{R}^{d}: x_{K} \text { is the barycenter of } K\right\}
$$

for some $\lambda>0$, then so is $T_{a}[h]$.

(3) (Higher regularity) If $h$ and a are smooth, so is $T_{a}[h]$.

Remark 1.15. In $d \leq 3$, our proof does not apply at the critical regularity $e \in \dot{H}^{\frac{d-4}{2}}$, since the possible error of (1.10) belongs only to the ill-behaved space $\dot{H}^{-\frac{3}{2}}$. However, under an extra smallness assumption for $\|a\|_{\dot{H}^{\frac{d-2}{2}}}$, the conclusion of Theorem 1.14 holds for $h \in \dot{H}^{\sigma-1}$ and $e \in \dot{H}^{\sigma}$ for the subcritical regularities $\sigma>1-\frac{d}{2}$; see Proposition 4.2 below.

As a consequence of Theorem 1.14, we have the following extension result for the YangMills initial data sets.

Theorem 1.16. For $d \geq 4$, let $K$ be a convex domain in $\mathbb{R}^{d}$, and let $(a, e)$ be an $\mathcal{H}^{\frac{d-2}{2}}$ Yang-Mills initial data set on $2 K \backslash \bar{K}$. Then there exists an $\mathcal{H}^{\frac{d-2}{2}}$ Yang-Mills initial data set $(\bar{a}, \bar{e})$ on $\mathbb{R}^{d} \backslash \bar{K}$ that coincides with $(a, e)$ on $2 K \backslash \bar{K}$ and obeys

$$
\begin{aligned}
\|\bar{a}\|_{\dot{H}^{\frac{d-2}{2}}\left(\mathbb{R}^{d} \backslash \bar{K}\right)} & \lesssim L(K) \\
\|\bar{e}\|_{\dot{H}^{\frac{d-4}{2}}\left(\mathbb{R}^{d} \backslash \bar{K}\right)} & \lesssim\|a\|_{\dot{H}^{\frac{d-2}{2}}(2 K \backslash \bar{K})}, \\
& \left\|_{\dot{H}} \frac{d-2}{2}{ }_{(2 K \backslash \bar{K})}, L(K)\right\| e \|_{\dot{H}^{\frac{d-4}{2}}(2 K \backslash \bar{K})} .
\end{aligned}
$$

It can be arranged so that the association $(a, e) \mapsto(\bar{a}, \bar{e})$ is equivariant under constant gauge transformations, i.e., $(A d(O) a, A d(O) e) \mapsto(A d(O) \bar{a}, A d(O) \bar{e}))$ for each $O \in \mathbf{G}$. Moreover, if $(a, e)$ is smooth, then so is $(\bar{a}, \bar{e})$.

At this point, it is useful to introduce a suitable generalization of local energy for initial data sets at the optimal $L^{2}$-Sobolev regularity. For $d \geq 4$ even, we make a gauge-invariant definition

$$
\mathcal{E}_{U}^{\frac{d-2}{2}}(a, e)=\left\|\left(\mathbf{D}^{(a)}\right)^{\left(\frac{d-2}{2}\right)}(F[a], e)\right\|_{L^{2}(U)}^{2}+\|(F[a], e)\|_{L^{\frac{d}{2}}(U)}^{2} .
$$

Note that this is equivalent to the energy when $d=4$. For $d \geq 4$ odd, there is a nuisance that the optimal $L^{2}$-Sobolev regularity involves a fractional derivative. Here, we take an easy way out, and make a gauge-dependent definition in this case:

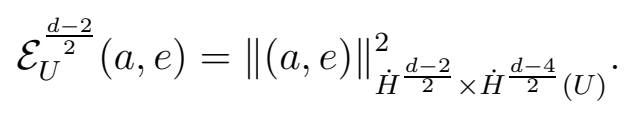

Let $\epsilon_{*}>0$. For $X=B_{R}$ or $\mathbb{R}^{d}$, we define the notion of the (inner) critical $L^{2}$-Sobolev concentration scale with threshold $\epsilon_{*}$ as follows:

$$
r_{c}^{\epsilon_{*}}=r_{c}^{\epsilon_{*}}[a, e]=\sup \left\{r>0: \mathcal{E}_{X \cap B_{r}(x)}^{\frac{d-2}{2}}(a, e) \leq \epsilon_{*}^{2} \text { for all } x \in X\right\},
$$

When $d=4$, we call $r_{c}^{\epsilon_{*}}$ the energy concentration scale with threshold $\epsilon_{*}$. 
Combining Theorem 1.16 with Uhlenbeck's lemma, we also obtain the following excisionand-extension result.

Theorem 1.17. Let $(a, e)$ be an $\mathcal{H}_{\text {loc }}^{\frac{d-2}{2}}$ Yang-Mills initial data set on $X=B_{R}\left(\right.$ resp. $\left.X=\mathbb{R}^{d}\right)$ with critical $L^{2}$-Sobolev concentration scale (with threshold $\epsilon_{*}$ ) at most $r_{c}$. Consider a ball $B_{r}(x)$ with radius $r<10 r_{c}$ and $x \in X$. For $\epsilon_{*}>0$ sufficiently small (as a universal constant), the following statements hold.

(1) To $(a, e)$, we associate $(\tilde{a}, \tilde{e}, O) \in \mathcal{H}^{\frac{d-2}{2}}\left(\mathbb{R}^{d}\right) \times \mathcal{G}^{\frac{d}{2}}\left(B_{r}(x) \cap X\right)$ such that $(\tilde{a}, \tilde{e})$ is gauge equivalent to $(a, e)$ on $B_{r}(x) \cap X$, i.e.,

$$
(\tilde{a}, \tilde{e})=\left(A d(O) a-O_{; x}, A d(O) e\right) \quad \text { in } B_{r}(x) \cap X .
$$

Moreover, $(\tilde{a}, \tilde{e})$ and $O$ obey the bounds

$$
\begin{aligned}
\|(\tilde{a}, \tilde{e})\|_{\dot{H}^{\frac{d-2}{2}} \times \dot{H}^{\frac{d-4}{2}}}^{2}+r^{-(d-2)}\|\tilde{a}\|_{L^{2}}^{2}+r^{-(d-4)}\|\tilde{e}\|_{L^{2}}^{2} & \lesssim \mathcal{E}_{B_{r}(x) \cap X}^{\frac{d-2}{2}}(a, e), \\
\left\|O_{; x}\right\|_{\dot{H}^{\frac{d-2}{2}}\left(B_{r}(x) \cap X\right)} & \lesssim\|a\|_{\dot{H}^{\frac{d-2}{2}}\left(B_{r}(x) \cap X\right)} .
\end{aligned}
$$

When $d$ is odd, $O$ is a constant gauge transformation. If $(a, e)$ is smooth, then so are $(\tilde{a}, \tilde{e})$ and $O$.

(2) Let $\left\{\left(a^{n}, e^{n}\right)\right\}$ be a sequence of $\mathcal{H}^{\frac{d-2}{2}}$ Yang-Mills initial data sets on $B_{r}(x) \cap X$ such that $\left(a^{n}, e^{n}\right) \rightarrow(a, e)$ in $H^{\frac{d-2}{2}} \times H^{\frac{d-4}{2}}\left(B_{r}(x) \cap X\right)$. Let $\left(\tilde{a}^{n}, \tilde{e}^{n}, O^{n}\right)$ be giverla by (1) from $\left(a^{n}, e^{n}\right)$. Then after passing to a subsequence and suitably conjugating each $\left(\tilde{a}^{n}, \tilde{e}^{n}, O^{n}\right)$ with a constant gauge transformation, we have

$$
\left(\tilde{a}^{n}, \tilde{e}^{n}\right) \rightarrow(\tilde{a}, \tilde{e}) \text { in } H^{\frac{d-2}{2}} \times H^{\frac{d-4}{2}}\left(\mathbb{R}^{d}\right), \quad O^{n} \rightarrow O \text { in } H^{\frac{d}{2}}\left(B_{r}(x) \cap X\right) .
$$

Remark 1.18. Theorems 1.16 and 1.17 have a similar flavor to the so-called initial data gluing procedure in general relativity [5, 6, 7, which is a method to remove an error in the constraint equation while keeping physical space localization properties. See [19] for an adaptation of this procedure for the Maxwell-Klein-Gordon constraint equation at the critical regularity, which had a similar role as Theorems 1.16 and 1.17 in the present paper. We also note that an initial data extension theorem, analogous to Theorem 1.16, was recently proved for the vacuum Einstein equation at the $L^{2}$-curvature regularity [8, 9].

As is evident from (2), it is natural to view the association $(a, e) \mapsto(\tilde{a}, \tilde{e}, O)$ in $(1)$ as defined up to a constant gauge transformation.

1.7. Local theory in an arbitrary topological class. We present the third set of results of this paper, which concern local theory of (1.9) for arbitrary $\mathcal{H}_{l o c}^{\frac{d-2}{2}}$ initial data set. The main local well-posedness results in the temporal gauge (Theorems 1.22 and 1.27) are proved as consequences of the finite speed of propagation property of (1.9), the results in Section 1.6 and small data well-posedness results [21, 29].

We start with a (rather general) basic definition of a solution.

Definition 1.19. (1) An $\mathcal{H}_{\text {loc }}^{\frac{d-2}{2}}$ connection in an open set $\mathcal{O} \subseteq \mathbb{R}^{1+d}$ is a connection $\mathbf{D}=$ $\mathrm{d}+A$ satisfying

$$
\left(A, \partial_{t} A\right) \in C_{t} H_{l o c}^{\frac{d-2}{2}} \times C_{t} H_{l o c}^{\frac{d-4}{2}}(\mathcal{O}) .
$$

\footnotetext{
${ }^{2}$ Note that the hypothesis on the critical $L^{2}$-Sobolev concentration scale is satisfied for large enough $n$.
} 
(2) An $\mathcal{H}^{\frac{d-2}{2}}$ solution for the hyperbolic Yang-Mills equation (1.9) in $\mathcal{O}$ is an $\mathcal{H}_{l o c}^{\frac{d-2}{2}}$ connection $\mathbf{D}=\mathrm{d}+A$ in $\mathcal{O}$ which is the limit of regular solutions in the topology $C_{t} H_{l o c}^{\frac{d-2}{2}} \times C_{t} H_{l o c}^{\frac{d-4}{2}}(\mathcal{O})$.

It is straightforward to see that the set of $\mathcal{H}_{l o c}^{\frac{d-2}{2}}$ solutions is closed with respect to the $C_{t} H_{l o c}^{\frac{d-2}{2}} \times C_{t} H_{l o c}^{\frac{d-4}{2}}$ topology.

Next, we formulate the notion of gauge covariance of $\mathcal{H}_{l o c}^{\frac{d-2}{2}}$ connections, as follows:

Definition 1.20. (1) A regular gauge transformation in an open set $\mathcal{O} \subseteq \mathbb{R}^{1+d}$ is a map $O: \mathcal{O} \rightarrow \mathbf{G}$ with the regularity properties $O_{; t, x} \in C_{t} H_{l o c}^{N}$.

(2) An admissible gauge transformation in $\mathcal{O}$ is a map $O: \mathcal{O} \rightarrow \mathbf{G}$ with the regularity properties $O_{; t, x} \in C_{t} H_{l o c}^{\frac{d-2}{2}}$.

(3) We say that two $\mathcal{H}^{\frac{d-2}{2}}$ connections $A^{(1)}$ and $A^{(2)}$ in $\mathcal{O}$ are gauge equivalent if there exists an admissible gauge transformation $O$ in $\mathcal{O}$ such that $A_{j}^{(2)}=A d(O) A_{j}^{(1)}-O_{; j}$.

Any admissible gauge transformation may be approximated by regular gauge transformations in $C_{t} H_{l o c}^{\frac{d}{2}}$ (the proof is a straightforward variant of Lemma 3.2 below, and is left to the reader). As a consequence, if $A$ and $A^{\prime}$ are gauge equivalent $\mathcal{H}^{\frac{d-2}{2}}$ connections in $\mathcal{O}$, $A$ is a $\mathcal{H}^{\frac{d-2}{2}}$ solution to (1.9) if and only if $A^{\prime}$ is. Moreover, the class of gauge-equivalent connections is closed:

Proposition 1.21. The class $[A]$ of gauge-equivalent $\mathcal{H}^{\frac{d-2}{2}}$ connections is closed in the topology $C_{t} H_{l o c}^{\frac{d-2}{2}} \times C_{t} H_{l o c}^{\frac{d-4}{2}}(\mathcal{O})$

With the basic notion of a solution in our hands, we are ready to discuss the local theory of (1.9) for $\mathcal{H}_{l o c}^{\frac{d-2}{2}}$ initial data sets. Given a subset $X$ of $\mathbb{R}^{d}$ and a time interval $I$, denote by $\mathcal{D}_{I}(X)$ the future domain of dependence of $X$, intersected with $I \times \mathbb{R}^{d}$ :

$$
\mathcal{D}_{I}(X)=\left\{(t, x) \in[0, \infty) \times \mathbb{R}^{d}: B_{t}(x) \subseteq X\right\} \cap I \times \mathbb{R}^{d} .
$$

In [21], global well-posedness of (1.9) in the temporal gauge for small $\dot{\mathcal{H}}^{\frac{d-2}{2}}$ data on $\mathbb{R}^{d}$ was proved for dimensions $d \geq 4$ (see Theorem 5.2 below). Combined with the excision-andextension result in Section 1.6 and the finite speed of propagation property in the temporal gauge, we obtain:

Theorem 1.22 (Local well-posedness at optimal regularity, $d \geq 4$ ). For $d \geq 4$, there exists a dimensional constant $\epsilon_{*}>0$ such that the Yang-Mills equation in the temporal gauge is locally well-posed on the time interval of length $r_{c}^{\epsilon_{*}}=r_{c}^{\epsilon_{*}}[a, e]$ for initial data $(a, e) \in \mathcal{H}_{l o c}^{\frac{d-2}{2}}(X)$ for $X=B_{R}$ or $\mathbb{R}^{d}$. More precisely, the following statements hold.

(1) (Regular data) Let $(a, e)$ be a smooth Yang-Mills initial data set on $X$. Then there exists a unique smooth solution $A_{t, x}$ to the Yang-Mills equation in the temporal gauge on $\mathcal{D}_{\left[0, r_{c}\right)}(X)$ such that $\left(A_{j}, F_{0 j}\right) \uparrow_{\{t=0\}}=\left(a_{j}, e_{j}\right)$.

\footnotetext{
${ }^{3}$ The exposition of [21] is focused on the case $d=4$, but the proof extends in a straightforward manner to $d \geq 4$.
} 
(2) (Rough data) Let $\mathcal{H}_{l o c, r_{c}}^{\frac{d-2}{2}}(X)$ be the class of $\mathcal{H}_{l o c}^{\frac{d-2}{2}}(X)$ Yang-Mills initial data sets with concentration scale $\geq r_{c}$, topologized with the norm

$$
\|(a, e)\|_{\mathcal{H}_{l o c, r_{c}}^{\frac{d-2}{2}}(X)}=\sup _{x \in X}\|(a, e)\|_{\dot{H}^{\frac{d-2}{2}} \times \dot{H}^{\frac{d-4}{2}}\left(B_{r_{c}}(x) \cap X\right)} .
$$

Then the data-to-solution map admits a continuous extension

$$
\mathcal{H}_{l o c, r_{c}}^{\frac{d-2}{2}}(X) \ni(a, e) \mapsto\left(A_{x}, \partial_{t} A_{x}\right) \in C_{t} \mathcal{H}_{l o c, r_{c}}^{\frac{d-2}{2}}\left(\mathcal{D}_{\left[0, r_{c}\right)}(X)\right) .
$$

(3) (A-priori bound) The solution defined as above obeys the a-priori bound

$$
\left\|\left(A, \partial_{t} A\right)\right\|_{L^{\infty}\left(H^{\frac{d-2}{2}} \times H^{\frac{d-4}{2}}\right)\left(\mathcal{D}_{\left[0, r_{c}\right)}\left(B_{R^{\prime}}(x)\right)\right)} \lesssim\|(a, e)\|_{H^{\frac{d-2}{2}} \times H^{\frac{d-4}{2}}\left(B_{R^{\prime}}(x)\right)}
$$

for any $B_{R^{\prime}}(x) \subseteq X$.

The temporal gauge solution given by Theorem 1.22 represents any $\mathcal{H}_{l o c}^{\frac{d-2}{2}}$ solution in the sense of Definition 1.19.

Theorem 1.23. Any $\mathcal{H}_{\text {loc }}^{\frac{d-2}{2}}$ solution to the hyperbolic Yang-Mills equation in $\mathcal{D}_{I}(X)$ (where $X=B_{R}$ or $\mathbb{R}^{d}$ ) can be put into the temporal gauge.

When $X=\mathbb{R}^{d}$, we say that $A$ is a $\mathcal{H}^{\frac{d-2}{2}}$ solution to the hyperbolic Yang-Mills equation in $I \times \mathbb{R}^{d}$ if it is an $\mathcal{H}_{l o c}^{\frac{d-2}{2}}$ solution, and moreover satisfies the following condition for every $t \in I$ :

$$
\mathcal{E}_{\mathbb{R}^{d}}^{\frac{d-2}{2}}\left(A_{x}(t), F_{0 x}(t)\right)<\infty .
$$

By Uhlenbeck's lemma and Theorem 1.22. (3), (1.21) holds for every $t \in I$ if it holds for its data $(a, e)$ at some $t \in I$. For such a solution, the topological class of $A_{x}(t)$ is preserved under the hyperbolic Yang-Mills evolution.

Proposition 1.24. Let $A$ be an $\mathcal{H}^{\frac{d-2}{2}}$ solution to $(1.9)$ in $I \times \mathbb{R}^{4}$. Then $\left[A_{x}(t)\right]$ is constant in $t$.

The temporal gauge is convenient in order to deal with causality, but it lacks good dispersive bounds in contrast to the caloric gauge [21] (cf. also the small data result in the Coulomb gauge in [15]). In a different global gauge, the caloric gauge regularity may be patched up, as the following sample result demonstrates:

Theorem 1.25. Let $A$ be an $\mathcal{H}_{\text {loc }}^{\frac{d-2}{2}}$ solution to (1.9) in $\mathcal{D}_{\left[0, r_{c}\right)}\left(B_{R}\right)$, whose initial data set has critical $L^{2}$-Sobolev concentration scale $\geq r_{c}$ with sufficiently small $\epsilon_{*}>0$. In a suitable global gauge in $D=\left[0, r_{c}\right) \times B_{R-4 r_{c}}$, the solution obeys

$$
\left\|\nabla A_{x}\right\|_{L^{\infty} \dot{H} \frac{d-4}{2}^{\frac{d-4}{2}}}+\left\|\square A_{x}\right\|_{\ell^{1} L^{2} \dot{H}^{\frac{d-5}{2}(D)}}+\left\|\nabla A_{0}\right\|_{\ell^{1} L^{2} \dot{H} \frac{d-3}{2}_{(D)}} \lesssim_{\epsilon_{*}, \frac{R}{r_{c}}} 1 .
$$

Remark 1.26. The restriction to $\left[0, r_{c}\right) \times B_{R-4 r_{c}}$ instead of $\mathcal{D}_{\left[0, r_{c}\right)\left(B_{R}\right)}$ is enforced merely to avoid technical issues near the boundary, and may be removed if desired. We do not pursue this improvement, since Theorem 1.25] suffices for our application in [22].

Finally, we discuss application of our techniques to the case of $d=3$. For $X=B_{R}$ or $\mathbb{R}^{3}$, we topologize the space $\mathcal{H}_{l o c}^{\sigma}(X)$ with the norm

$$
\|(a, e)\|_{\mathcal{H}_{l o c}^{\sigma}(X)}=\sup _{x \in X}\|(a, e)\|_{H^{\sigma} \times H^{\sigma-1}\left(B_{1}(x) \cap X\right)}
$$


From the small data local well-posedness result of Tao [29], we obtain the following large data result:

Theorem 1.27 (Local well-posedness in the temporal gauge, $d=3$ ). Let $\sigma>\frac{3}{4}$. The YangMills equation in the temporal gauge is locally well-posed for initial data $(a, e) \in \mathcal{H}_{\text {loc }}^{\sigma}\left(\mathbb{R}^{3}\right)$ on a time interval of length $\geq T\left(\|(a, e)\|_{\mathcal{H}_{\text {loc }}^{\sigma}}\right)$.

Moreover, the techniques of this paper lead to an alternative proof of the classical result of Klainerman-Machedon [11]:

Theorem 1.28. The Yang-Mills equation in the temporal gauge is globally well-posed for initial data $(a, e) \in \mathcal{H}_{\text {loc }}^{1}\left(\mathbb{R}^{3}\right)$.

An advantage of the present approach is that the delicate issue of boundary values on spacetime cones (i.e., the domains of dependence of balls) is avoided by the robust excisionand-extension procedure. We note that yet another proof of Theorem 1.28 relying on a global gauge defined by the Yang-Mills heat flow (a subcritical version of the caloric gauge we use in the present series [20, 21, 22]) was given by the first author [17, 18].

1.8. Topological classes, instantons and harmonic Yang-Mills connections on $\mathbb{R}^{4}$. In this subsection, we restrict to the energy critical dimension $d=4$, and discuss the relationship between the topological class of a connection $a$ on $\mathbb{R}^{4}$ and its static energy

$$
\mathcal{E}_{e}(a)=\mathcal{E}_{\mathbb{R}^{4}}(a, 0)=\frac{1}{2} \int_{\mathbb{R}^{4}}\left\langle F_{j k}[a], F^{j k}[a]\right\rangle \mathrm{d} x .
$$

Recall that each topological class $[a]$ of finite energy connections form a path-connected component in the $\dot{H}^{1}$ distance up to gauge transformations (Section 1.4). We may therefore look for an absolute minimizer of $\mathcal{E}_{e}(a)$ in each topological class; such a connection is called an instanton]. More generally, we refer to a critical point of (1.23) as a harmonic Yang-Mills connection.

Such connections are clearly static solutions to both the Yang-Mills heat flow and the hyperbolic Yang-Mills equation, and hence obstructions to convergence of solutions to the trivial connection (as well as scattering). Moreover, these connections may also arise as "bubbles" near the singularity of a dynamic solution. Therefore, knowledge of the energies of the harmonic Yang-Mills connections is necessary for determining the precise threshold energy in the Threshold Theorem, both for the Yang-Mills heat flow [20] and for the hyperbolic Yang-Mills equation 22.

We open our discussion with the important special case $\mathbf{G}=S U(2)$. The corresponding Lie algebra $\mathfrak{g}=s u(2)$ consists of $2 \times 2$ complex anti-hermitean matrices with zero trace. We furthermore assume that the $A d$-invariant inner product on $\mathfrak{g}$ takes the form

$$
\langle A, B\rangle=-\operatorname{tr}(A B) .
$$

In fact, as all $A d$-invariant inner products on $\mathfrak{g}$ are positive multiples of each other, there is no loss of generality.

\footnotetext{
${ }^{4}$ Usually, one also distinguishes between an instanton and an anti-instanton, depending on whether the curvature is self- or anti-self-dual. Here, we make no such distinction.
} 
In this case, the topological classes of finite energy connections are classified by the second Chern number $c_{2}$, which takes the explicit form (via the Chern-Weil theory)

$$
c_{2}=\frac{1}{8 \pi^{2}} \int_{\mathbb{R}^{4}} \operatorname{tr}(F[a] \wedge F[a]) .
$$

For any finite energy connection $a$, the second Chern number $c_{2}$ is an integer; in fact, it equals the degree of the 0-homogeneous map $O$ (defined using the homeomorphism $S U(2) \simeq \mathbb{S}^{3}$ ) in Theorem 1.5. A simple algebraic manipulation using the Hodge star operator 5 * shows that

$$
\begin{aligned}
\left\langle F_{j k}[a], F^{j k}[a]\right\rangle & =-\star 2 \operatorname{tr}(F \wedge \star F) \\
& =-\star \operatorname{tr}((F \pm \star F) \wedge \star(F \pm \star F)) \pm 2 \star \operatorname{tr}(F \wedge F) \\
& =\frac{1}{2}\langle F \pm \star F, F \pm \star F\rangle \pm 2 \star \operatorname{tr}(F \wedge F) .
\end{aligned}
$$

Note that the first term on the last line is nonnegative. Integrating over $\mathbb{R}^{4}$, we obtain the Bogomoln'yi bound

$$
\mathcal{E}_{e}(a) \geq 8 \pi^{2}\left|c_{2}\right|
$$

The equality holds (in which case, $a$ is an instanton) if and only if $F=\mp \star F$, where \pm is the sign of $c_{2}$. We call such a connection anti-self or self dual, respectively. There is a beautiful theory due to Atiyah-Drinfeld-Hitchin-Manin [1], which gives an explicit construction of all anti-self dual (resp. self-dual) connections with $c_{2}>0$ (resp. $\left.c_{2}<0\right)$. In particular, we have:

Theorem 1.29 ([1]). For any $\kappa \in \mathbb{Z}$, there exists an instanton with $c_{2}=-\kappa$ and energy $8 \pi^{2}|\kappa|$.

However, the instantons do not tell the full story. It is known that there also exist nontrivial harmonic Yang-Mills connections which are not self or anti-self dual [27, 3, 25, 24]. Nevertheless, by the recent result of Gursky-Kelleher-Streets [10], they must have energy at least $16 \pi^{2}$ more than the Bogomoln'yi bound:

Theorem 1.30 ([10, Corollary 1.2]). Any harmonic Yang-Mills connection on $\mathbb{R}^{4}$ either has energy equal to $8 \pi^{2}\left|c_{2}\right|$, or has energy at least $8 \pi^{2}\left|c_{2}\right|+16 \pi^{2}$.

In conclusion, we see that: Any nontrivial harmonic SU(2) Yang-Mills connection either has energy at least $16 \pi^{2}$, or it is an instanton with $c_{2}= \pm 1$ (a first instanton) with energy $8 \pi^{2}$. We call the first instanton alternatively as the ground state (as it has the lowest nontrivial energy), and refer to its energy as the ground state energy $E_{G S}$.

We now turn to the general case when $\mathbf{G}$ is a compact Lie group, for which our goal is to establish a similar conclusion. Consider $f_{2}(\cdot, \cdot)=-\langle\cdot, \cdot\rangle$, which is a symmetric $A d$-invariant bilinear function, and the corresponding characteristic class (cf. Section [1.2).

$$
-\langle F[a] \wedge F[a]\rangle=-\left\langle F_{i j}[a], F_{k \ell}[a]\right\rangle \mathrm{d} x^{i} \wedge \mathrm{d} x^{j} \wedge \mathrm{d} x^{k} \wedge \mathrm{d} x^{\ell} .
$$

The characteristic number

$$
\chi=\int_{\mathbb{R}^{4}}-\langle F[a] \wedge F[a]\rangle
$$

\footnotetext{
${ }^{5}$ To define $\star$, we use the standard inner product on 2 -forms such that $\left\{\mathrm{d} x^{j} \wedge \mathrm{d} x^{k}: j<k\right\}$ is an orthonormal basis.

${ }^{6}$ Note that [10, Corollary 1.2] is stated on $\mathbb{S}^{4}$, but the same conclusion holds on $\mathbb{R}^{4}$ by conformal invariance of the harmonic Yang-Mills equation and $\mathcal{E}_{e}$. Moreover, to compare the results, recall that $\mathcal{E}_{e}(a)=\frac{1}{2}\|F[a]\|_{L^{2}}^{2}$.
} 
is determined by the topological class $[a]$, by Corollary 1.10, Moreover, the same algebra as in (1.25) leads to:

Lemma 1.31. Let $\mathbf{G}$ be a compact Lie group. For any finite energy connection a on a $\mathbf{G}$-bundle on $\mathbb{R}^{4}$, we have the pointwise bound

$$
\frac{1}{2}\left\langle F_{j k}[a], F^{j k}[a]\right\rangle \geq|\langle F[a] \wedge F[a]\rangle|,
$$

and the corresponding integrated bound

$$
\mathcal{E}_{e}(a) \geq|\chi|
$$

Note that when $\mathbf{G}$ is commutative, then the harmonic Yang-Mills connections are nothing else than the harmonic 2-forms; thus no nontrivial finite energy harmonic Yang-Mills connections exist. In the noncommutative case, we prove:

Theorem 1.32. Let $\mathbf{G}$ be a noncommutative compact Lie group. Let

$E_{G S}=\inf \left\{\mathcal{E}_{e}(a):\right.$ a is a nontrivial harmonic Yang-Mills connection on a $\mathbf{G}$-bundle on $\left.\mathbb{R}^{4}\right\}$.

Then the following statements hold.

(1) There exists a nontrivial harmonic Yang-Mills connection a such that $\mathcal{E}_{e}(Q)=E_{G S}<$ $\infty$.

(2) Let a be any nontrivial harmonic Yang-Mills connection. Then either $\mathcal{E}_{e}(a) \geq 2 E_{G S}$, or

$$
|\chi|=\mathcal{E}_{e}(a) \geq E_{G S}
$$

We call $E_{G S}$ the ground state energy, and a harmonic Yang-Mills connection $Q$ attaining this energy a ground state.

The proof of Theorem 1.32 combines well-known results concerning the structure of a compact Lie group and the preceding analysis in the case $\mathbf{G}=S U(2)$; it is provided in Section 6 .

Acknowledgments. S.-J. Oh was supported by the Miller Research Fellowship from the Miller Institute, UC Berkeley and the TJ Park Science Fellowship from the POSCO TJ Park Foundation. D. Tataru was partially supported by the NSF grant DMS-1266182 as well as by a Simons Investigator grant from the Simons Foundation.

\section{Notation AND CONVENTIONS}

Here we collect some notation and conventions used in this paper.

- We employ the usual asymptotic notation $A \lesssim B$ to denote $A \leq C B$ for some implicit constant $C>0$. The dependence of $C$ on various parameters is specified by subscripts.

- Throughout the paper, we omit the dependence of constants on the dimension $d$. In particular, by a universal constant, we mean a constant that depends only on $d$.

- We call a bounded open subset $U$ of $\mathbb{R}^{d}$ a domain. For $\lambda>0, \lambda U$ is defined to be rescaling of $U$ by $\lambda$ centered at the barycenter of $U$. For any $r>0$ and $x \in \mathbb{R}^{d}, B_{r}(x)$ is the ball of radius $r$ centered at $x$. When $(x)$ is omitted, the center is taken to be the origin 0 . 
- We use the notation $\partial$ (without sub- or superscripts) for the spatial gradient $\partial=$ $\left(\partial_{1}, \partial_{2}, \ldots, \partial_{d}\right)$, and $\nabla$ for the spacetime gradient $\nabla=\left(\partial_{0}, \partial_{1}, \ldots, \partial_{d}\right)$. We write $\partial^{(n)}$ (resp. $\left.\nabla^{(n)}\right)$ for the collection of $n$-th order spatial (resp. spacetime) derivatives, and $\partial^{(\leq n)}$ (resp. $\nabla^{(\leq n)}$ ) for those up to order $n$.

- The $n$-th homogeneous $L^{p}$-Sobolev space for functions from $\mathbb{R}^{d}$ into a normed vector space $V$ is denoted by $\dot{W}^{n, p}\left(\mathbb{R}^{d} ; V\right)$. In the special case $p=2$, we write

$$
\dot{H}^{n}\left(\mathbb{R}^{d} ; V\right)=\dot{W}^{n, 2}\left(\mathbb{R}^{d} ; V\right) .
$$

The inhomogeneous counterparts are denoted by $W^{n, p}\left(\mathbb{R}^{d} ; V\right)$ and $H^{n}\left(\mathbb{R}^{d} ; V\right)$, respectively. The Lebesgue spaces (i.e., when $n=0)$ are denoted by $L^{p}\left(\mathbb{R}^{d} ; V\right)$.

- The mixed spacetime norm $L_{t}^{q} \dot{W}_{x}^{n, r}$ of functions on $\mathbb{R}^{1+d}$ is often abbreviated as $L^{q} \dot{W}^{n, r}$.

- Given a function space $X$ (on either $\mathbb{R}^{d}$ or $\mathbb{R}^{1+d}$ ), we define the space $\ell^{p} X$ by

$$
\|u\|_{\ell^{p} X}^{p}=\sum_{k}\left\|P_{k} u\right\|_{X}^{p}
$$

(with the usual modification for $p=\infty)$, where $P_{k}(k \in \mathbb{Z})$ are the usual LittlewoodPaley projections to dyadic frequency annuli.

- Generally, a function space on an open subset $U \subseteq \mathbb{R}^{d}$ is defined by restriction, i.e., $\|u\|_{X(U)}=\inf \left\{\|\tilde{u}\|_{X}: \tilde{u} \in X, \tilde{u} \uparrow_{U}=u\right\}$. A similar convention applies for a function space on an open subset $\mathcal{O} \subseteq \mathbb{R}^{1+d}$.

According to this convention, the restriction of the homogeneous Sobolev norm $\dot{W}^{n, p}$ for $n \in \mathbb{N}, 1<p<\frac{d}{n}$ for a locally Lipschitz domain $U$ is characterized by

$$
\|u\|_{\dot{W}^{n, p}(U)} \simeq_{U}\left\|\partial^{(n)} u\right\|_{L^{p}(U)}+\|u\|_{L^{p^{*}(U)}}, \quad \text { where } \frac{d}{p^{*}}=\frac{d}{p}-n .
$$

Note, importantly, that the implicit constant is invariant under scaling. To distinguish this norm from the usual homogeneous Sobolev semi-norm, we introduce the notation $\stackrel{\circ}{W}^{n, p}(U)$ for a nonnegative integer $n$ and $p \in[1, \infty]$, and define $\|u\|_{\dot{W}^{n, p}(U)}=$ $\left\|\partial^{(n)} u\right\|_{L^{p}(U)}$.

- The local function space $X_{l o c}(U)$ is defined as

$$
X_{l o c}(U)=\bigcap_{B_{x}(r): \bar{B}_{x}(r) \subseteq U} X\left(B_{x}(r)\right) .
$$

\section{Connections With $L^{\frac{d}{2}}$-Curvature}

In this section, we prove the good global gauge theorems Theorems 1.4 and 1.5. Throughout the section, we let $d \geq 3$.

3.1. G-valued functions at critical regularity. We start by collecting some basic analytic facts concerning $\mathbf{G}$-valued functions at regularity $W^{k, \frac{d}{k}}$.

In what follows, we assume that $\mathbf{G}$ is a group of orthogonal matrices in $\mathbb{R}^{N \times N}$, equipped with the usual inner product $\langle A, B\rangle=\operatorname{tr} A B^{\dagger}$. Recall the standard fact that any compact Lie group $\mathbf{G}$ may be realized as such a matrix group, and the inner product on $\mathfrak{g}=T_{I d} \mathbf{G}$ is equivalent to the one induced from $\mathbb{R}^{N \times N}$. 
Let $U \subseteq \mathbb{R}^{d}$ be an open set, $k \in \mathbb{R}$ and $p \in[1, \infty]$. In Section 1.3 , we introduced

$$
\mathcal{G}^{k, p}(U)=\left\{O \in W^{k, p}\left(U ; \mathbb{R}^{N \times N}\right): O(x) \in \mathbf{G} \text { for a.e. } x \in U\right\} .
$$

Since $\mathbf{G}$ is compact, any $O \in \mathcal{G}^{k, p}(U)$ belongs to $L^{\infty}(U)$. When $U$ is a domain with locally Lipschitz boundary, an element $O \in \mathcal{G}^{k, p}(U)$ may be extended $\sqrt{7}$ to $\tilde{O} \in W^{k, p} \cap L^{\infty}\left(\mathbb{R}^{d}\right)$; see [28, §VI.3]. For a general irregular open set $U$, we instead use

$$
\mathcal{G}_{l o c}^{k, p}(U)=\left\{O \in W_{l o c}^{k, p}\left(U ; \mathbb{R}^{N \times N}\right): O(x) \in \mathbf{G} \text { for a.e. } x \in U\right\},
$$

for which the following extension property holds: For any ball $B \subseteq U$, there exists ${ }^{(B)} \tilde{O} \in$ $W^{k, p} \cap L^{\infty}\left(\mathbb{R}^{d}\right)$ such that ${ }^{(B)} \tilde{O}(x)=O(x)$ for a.e. $x \in B$.

In view of the applications to the hyperbolic Yang-Mills equation at the critical regularity, we consider the scale-invariant case $p=\frac{d}{k}>1$, which is subtle due to the fact that $H^{k, \frac{d}{k}} \not$ $L^{\infty}$, and thus $H^{k, \frac{d}{k}}$ is not an algebra. Nevertheless, as we will see, basic operations needed to define a G-bundle are still well-defined. To avoid technical issues, we focus on the case when $k$ is a positive integer. Of special importance is when $k=2$, which correspond to local gauge transformations in a bundle admitting a connection with $L^{\frac{d}{2}}$ curvature.

As a quick consequence of the extension properties mentioned above, we have the following multiplication lemma.

Lemma 3.1. Let $k$ be a positive integer, and let $U \subseteq \mathbb{R}^{d}$ be an open set. Then the pointwise multiplication map

$$
\mathcal{G}_{l o c}^{k, \frac{d}{k}}(U) \times \mathcal{G}_{l o c}^{k, \frac{d}{k}}(U) \ni\left(O_{1}, O_{2}\right) \mapsto O_{1} \cdot O_{2} \in \mathcal{G}_{l o c}^{k, \frac{d}{k}}(U)
$$

is continuous. If $U$ is a domain with a locally Lipschitz boundary, then the same conclusion holds for the space $\mathcal{G}^{k, \frac{d}{k}}(U)$.

Although multiplication is continuous, we remark that it utterly fails to be any more regular. This is in sharp contrast with the subcritical case $\mathcal{G}^{k, p}$ with $p>\frac{d}{k}$, in which multplication is smooth.

Proof. It suffices to consider the case when $U$ is a domain with a locally Lipschitz boundary (the other case follows by taking $U$ to be balls). Let $O_{1}, O_{2} \in \mathcal{G}^{k, \frac{d}{k}}(U)$, and consider their usual extensions outside $U$. Note that $O_{1} \cdot O_{2}$ is an $L_{l o c}^{1}$ function with values in $\mathbf{G}$ for a.e. $x \in U$, and belongs to $W^{k, \frac{d}{k}}(U)$ by the whole space estimate

$$
\left\|O_{1} \cdot O_{2}\right\|_{W^{k, \frac{d}{k}}} \lesssim\left\|O_{1}\right\|_{L^{\infty}}\left\|O_{2}\right\|_{W^{k, \frac{d}{k}}}+\left\|O_{1}\right\|_{W^{k, \frac{d}{k}}}\left\|O_{2}\right\|_{L^{\infty}}
$$

To prove continuity, consider sequences $O_{1}^{n} \rightarrow O_{1}$ and $O_{2}^{n} \rightarrow O_{2}$ in $\mathcal{G}^{k, \frac{d}{k}}(U)$. We extend $O_{1}^{n}$ and $O_{2}^{n}$ to the whole space using the same extension operator as before, which insures $O_{1}^{n} \rightarrow O_{1}$ and $O_{2}^{n} \rightarrow O_{2}$ in $W^{k, \frac{d}{k}}\left(\mathbb{R}^{d} ; \mathbb{R}^{N \times N}\right)$. By the Leibniz rule and the Sobolev inequality, for any multi-index $\alpha$ of order $k$, we may show that

$$
\partial^{\alpha}\left(O_{1}^{n} \cdot O_{2}^{n}\right)-\left(\partial^{\alpha} O_{1}^{n}\right) \cdot O_{2}^{n}-O_{1}^{n} \cdot \partial^{\alpha} O_{2}^{n} \rightarrow \partial^{\alpha}\left(O_{1} \cdot O_{2}\right)-\left(\partial^{\alpha} O_{1}\right) O_{2}-O_{1} \partial^{\alpha} O_{2} \text { in } L^{\frac{d}{k}}
$$

By symmetry, it only remains to prove that $\left(\partial^{\alpha} O_{1}^{n}\right) \cdot O_{2}^{n} \rightarrow\left(\partial^{\alpha} O_{1}\right) \cdot O_{2}$ in $L^{\frac{d}{k}}$. Since $O_{2}^{n}$ is uniformly bounded, the problem is further reduced to showing that

$$
\left\|\partial^{\alpha} O_{1} \cdot\left(O_{2}^{n}-O_{2}\right)\right\|_{L^{\frac{d}{k}}} \rightarrow 0 .
$$

${ }^{7}$ We emphasize, however, that $\tilde{O}(x) \notin \mathbf{G}$ for $x \notin U$ in general. 
If this limit were not true, then there would exist a subsequence with no further subsequence converging to zero. However, $O_{2}^{n} \rightarrow O_{2}$ in $W^{k, \frac{d}{k}}$ implies a.e. convergence along a subsequence, along which the above limit holds by the dominated convergence theorem.

It is well-known that if $U$ is an open set with piecewise smooth boundary, then any $O \in \mathcal{G}^{2, \frac{d}{2}}(U)$ can be approximated by a sequence $O^{n} \in C^{\infty}(U ; \mathbf{G})$ in the $W^{2, \frac{d}{2}}\left(U ; \mathbb{R}^{N \times N}\right)$ topology [26]. We state here a technical refinement which allows us to localize the region where we perform the approximation (essentially from [31]). This version will be helpful for handling the extension problem to a G-valued map (not $\mathbb{R}^{N \times N}$-valued).

Lemma 3.2. Let $k$ be a positive integer. Let $U \subseteq \mathbb{R}^{d}$ be a domain with locally Lipschitz boundary, and let $O \in \mathcal{G}^{k, \frac{d}{k}}(U)$. If $V, W$ are (possibly empty) open sets in $\mathbb{R}^{d}$ such that $\bar{V} \cup \bar{W} \subseteq \bar{U}$ and $\bar{V} \cap \bar{W}=\emptyset$, then for every $\epsilon>0$ there exists $O^{\prime} \in \mathcal{G}^{k, \frac{d}{k}}(U)$ such that $O^{\prime} \uparrow_{V}=O \uparrow_{V}, O^{\prime} \in C^{\infty}(W ; \mathbf{G})$ and $\left\|O^{\prime}-O\right\|_{W^{k, \frac{d}{k}}\left(U ; \mathbb{R}^{N \times N}\right)}<\epsilon$.

We recover the usual approximation result by setting $V=\emptyset$ and $W=U$. As a consequence, for a general open set $U$, any $O \in \mathcal{G}_{l o c}^{k, \frac{d}{k}}(U)$ can be approximated by $O^{n} \in C^{\infty}(B ; \mathbf{G})$ in the $W^{k, \frac{d}{k}}\left(B ; \mathbb{R}^{N \times N}\right)$-topology for any open ball $B \subseteq U$.

Proof. We may assume that $W \neq \emptyset$, as otherwise we may set $O^{\epsilon}=O$. By standard Sobolev extension, there exists $\tilde{O} \in W^{k, \frac{d}{k}}\left(\mathbb{R}^{d} ; \mathbb{R}^{N \times N}\right)$ such that $\tilde{O} \uparrow_{U}=O$. We introduce $\delta>0$ to be fixed later, and let $h: U \rightarrow[0,1]$ be a smooth function such that $h=0$ on $V$ and $h=1$ on $W$ (smooth Urysohn's lemma). Fix a smooth function $\zeta$ supported in the unit ball satisfying $\int \zeta=1$. We define $\tilde{O}^{\delta}: \mathbb{R}^{d} \rightarrow \mathbb{R}^{N \times N}$ by inhomogeneous mollification:

$$
\tilde{O}^{\delta}(x)=\int \zeta(y) \tilde{O}(x-\delta h(x) y) \mathrm{d} y .
$$

It is straightforward to verify that $\left\|\tilde{O}^{\delta}-\tilde{O}\right\|_{W^{k, \frac{d}{k}(U)}} \rightarrow 0$ as $\delta \rightarrow 0$, and also that $\tilde{O}^{\delta}$ is smooth on $W$. However, $\tilde{O}^{\delta}(x) \notin \mathbf{G}$ in general. To rectify this, we proceed as in [26].

Let $\tilde{\mathbf{G}} \subseteq \mathbb{R}^{N \times N}$ be a tubular neighborhood of $\mathbf{G}$, on which the nearset-point projection $\pi_{\mathbf{G}}: \tilde{\mathbf{G}} \rightarrow \mathbf{G}$ is well-defined as a smooth map. For $x \in U$, we wish to ensure that $\tilde{O}^{\delta}(x) \in \tilde{\mathbf{G}}$ for $\delta$ sufficiently small. Since $O(y) \in \mathbf{G}$ for a.e. $y \in U$, we have

$$
d\left(\tilde{O}^{\delta}(x), \mathbf{G}\right)^{d} \leq \frac{1}{\left|U \cap B_{\delta h(x)}(x)\right|} \int_{U \cap B_{\delta h(x)}(x)}\left|\tilde{O}^{\delta}(x)-O(y)\right|^{d} \mathrm{~d} y .
$$

By boundedness and the locally Lipschitz condition, $\left|U \cap B_{r}(x)\right| \gtrsim_{U, d} r^{d}$ for every $x \in \bar{U}$ and sufficiently small $r>0$. Moreover, by the Poincaré inequality $\|f\|_{L^{d}\left(B_{r}(x)\right)} \lesssim_{\zeta} r\|\partial f\|_{L^{d}\left(B_{r}(x)\right)}$ for $f$ satisfying $\int \zeta(y) f(x+r y) \mathrm{d} y=0$, we have

$$
d\left(\tilde{O}^{\delta}(x), \mathbf{G}\right)^{d} \lesssim_{U, \zeta, d} \int_{B_{\delta h(x)}(x)}|\partial \tilde{O}(y)|^{d} \mathrm{~d} y .
$$

By compactness of $\bar{U}$, the RHS goes to 0 uniformly as $\delta \rightarrow 0$, so that $\tilde{O}^{\delta}(x) \in \tilde{\mathbf{G}}$.

Define $O^{\prime}=\pi_{\mathrm{G}} \circ \tilde{O}^{\delta} \uparrow_{U}$. It is now straightforward to show that $O^{\prime}$ obeys the desired properties once we fix $\delta>0$ small enough (depending on $\epsilon$ ). 
As a consequence of the approximation property, we now show that pointwise inversion is well-defined as a continuous map $\mathcal{G}_{l o c}^{k, \frac{d}{k}}(U) \rightarrow \mathcal{G}_{l o c}^{k, \frac{d}{k}}(U)$.

Lemma 3.3. Let $k$ be a positive integer, and let $U \subseteq \mathbb{R}^{d}$ be an open set. Then the pointwise inversion map

$$
\mathcal{G}_{\text {loc }}^{k, \frac{d}{k}}(U) \ni O \mapsto O^{-1} \in \mathcal{G}_{l o c}^{k, \frac{d}{k}}(U)
$$

is continuous. Moreover, the usual differentiation rule $\partial_{x} O^{-1}=-O^{-1} \partial_{x} O O^{-1}$ holds for $O \in \mathcal{G}_{l o c}^{k, \frac{d}{k}}(U)$. If $U$ is a domain with a locally Lipschitz boundary, then the same conclusion holds for the space $\mathcal{G}^{k, \frac{d}{k}}(U)$.

Proof. As before, we only consider the case when $U$ is a domain with a locally Lipschitz boundary. For simplicity, we only treat the case $k=1$; the higher $k$ 's are handled similarly. Given $O \in \mathcal{G}^{1, d}(U)$, let $O^{n} \rightarrow O$ be a smooth approximation sequence in $\mathcal{G}^{1, d}(U)$ given by Lemma 3.2 (with $V=\emptyset$ and $W=U$ ). By passing to a subsequence, we may assume that $O^{n} \rightarrow O$ a.e. in $U$ as well; hence $\left(O^{n}\right)^{-1} \rightarrow O^{-1}$ in $U$. Moreover, by the usual differentiation formula in the smooth case,

$$
\partial_{x}\left(O^{n}\right)^{-1}=-\left(O^{n}\right)^{-1} \partial_{x} O^{n}\left(O^{n}\right)^{-1} .
$$

By the dominated convergence theorem, $\partial_{x}\left(O^{n}\right)^{-1}$ is Cauchy in $W^{1, d}\left(U ; \mathbb{R}^{N \times N}\right)$, so that $O^{-1} \in \mathcal{G}^{1, d}(U)$. Moreover, the formula

$$
\partial_{x} O^{-1}=-O^{-1} \partial_{x} O O^{-1}
$$

is justified for $O \in \mathcal{G}^{1, d}(U)$. By a similar argument using the dominated convergence theorem applied to an arbitrary sequence $O^{n} \rightarrow O$ in $\mathcal{G}^{1, d}(U)$. the continuity property also follows.

Next, from the approximation property and Lemma 3.3, it follows that the usual operations involving $\mathcal{G}_{l o c}^{k, \frac{d}{k}}(U)$ and $W_{l o c}^{k^{\prime}, \frac{d}{k}}(U ; \mathfrak{g})$ are continuous.

Lemma 3.4. Let $k$ be a positive integer, and let $U \subseteq \mathbb{R}^{d}$ be an open set.

(1) The operations $O \mapsto O_{; x}=\partial_{x} O O^{-1}$ and $O \mapsto O_{; x}^{-1}=-O^{-1} \partial_{x} O$ are continuous as mappings $\mathcal{G}_{\text {loc }}^{k, \frac{d}{k}}(U) \rightarrow W_{l o c}^{k-1, \frac{d}{k}}(U ; \mathfrak{g})$.

(2) For any integer $0 \leq k^{\prime} \leq k$, the operation $(O, B) \mapsto A d(O) B=O B O^{-1}$ is continuous as a mapping $\mathcal{G}_{l o c}^{k, \frac{d}{k}}(U) \times W_{l o c}^{k^{\prime}, \frac{d}{k}}(U ; \mathfrak{g}) \rightarrow W_{l o c}^{k^{\prime}, \frac{d}{k}}(U ; \mathfrak{g})$.

(3) If $O, O_{1}, O_{2} \in \mathcal{G}_{l o c}^{k, \frac{d}{k}}(U)$ and $B \in W_{l o c}^{k^{\prime}, \frac{d}{k}}(U ; \mathfrak{g})$, then the following Leibniz rules hold:

$$
\begin{aligned}
\left(O_{1} O_{2}\right)_{; x} & =O_{1 ; x}+A d\left(O_{1}\right) O_{2 ; x} \\
\partial_{x}(A d(O) B) & =A d(O) \partial_{x} B+A d(O)\left[O_{; x}, B\right] .
\end{aligned}
$$

If $U$ has a locally Lipschitz boundary, then the same conclusion holds for the spaces $\mathcal{G}^{k, \frac{d}{k}}(U)$ and $W^{k^{\prime}, \frac{d}{k}}(U ; \mathfrak{g})$.

As before, the fact that these operations map into the right space is justified by using a smooth approximating sequence (Lemma 3.2), and then their continuity properties are proved in a similar manner. We omit the proof.

We end with an auxiliary lemma concerning the construction of a $\mathbf{G}$-valued function on an annulus with a prescribed normal derivative on the outer boundary. 
Lemma 3.5. Let $B$ be a $\mathfrak{g}$-valued function in $H^{\frac{d-3}{2}}\left(\mathbb{S}^{d-1}\right)$. There exists $O \in L^{\infty} \cap H^{\frac{d}{2}}\left(B_{1}\right)$, which depends continuously on $B$, such that

$$
\left(O, O_{; r}\right) \uparrow_{\{r=1\}}=(I d, B),
$$

where $O_{; r}=\frac{x^{j}}{|x|} O_{; j}$. A similar construction can be done in the exterior region $\mathbb{R}^{d} \backslash B_{1}$.

Proof. We first work on the annulus $B_{1} \backslash \overline{\frac{1}{2} B_{1}}$, which we view as the product space $\left(\frac{1}{2}, 1\right)_{r} \times \mathbb{S}_{\Theta}^{d-1}$ (note that the corresponding Lebesgue and Sobolev spaces are equivalent). We define $\varphi(r, \Theta$ ) to be Poisson semigroup $\varphi(r, \Theta)=e^{\sqrt{-\Delta_{\Theta}}(r-1)} B$, and define

$$
\Psi(r, \Theta)=(r-1) \varphi(r, \Theta),
$$

By the properties of the Poisson semigroup, observe that

$$
\Psi(r, \Theta)=(r-1) B(\Theta)+o_{r \rightarrow 1}(r-1) \quad \text { in } H^{\frac{d-3}{2}}\left(\mathbb{S}^{d-1}\right) .
$$

Moreover, $\Psi(r, \Theta) \in L^{\infty} \cap H^{\frac{d}{2}}\left(\left(\frac{1}{2}, 1\right) \times \mathbb{S}^{d-1}\right)$ and

$$
\|\Psi(r, \cdot)\|_{L^{\infty}(\mathbb{S} d-1)}=o_{r \rightarrow 1}(1)
$$

where the rate depends only on the right tail of the $H^{\frac{d-3}{2}}$ frequency envelope of $B$. Let

$$
O(r, \Theta)=\exp (\chi \Psi(r, \Theta)) \text {. }
$$

where $\chi=\chi(r)$ is a smooth radial function such that $\chi=0$ in $\left\{r<\frac{2}{3}\right\}$ and $\chi=1$ in $\left\{r>\frac{5}{6}\right\}$. Since $L^{\infty} \cap H^{\frac{d}{2}}$ is an algebra, and since $O=I d$ in $\left\{r<\frac{2}{3}\right\}$, it may be checked that $O \in L^{\infty} \cap H^{\frac{d}{2}}\left(B_{1}\right)$. Moreover, $\partial_{r} O(r, \Theta) O^{-1}(r, \Theta) \uparrow_{\{r=1\}}=\partial_{r} \Psi(r, \Theta) \uparrow_{\{r=1\}}=B(\Theta)$, as desired.

3.2. Patching procedures. Here we describe procedures for patching together local gauges to a global gauge, which is one of the main ingredients of the proof of the good global gauge theorems.

We consider three scenarios:

(1) Local gauges given on small (round) cubes $Q_{(\alpha)}$ covering a large (round) cube $Q_{R}$;

(2) Local gauges given on small balls $B_{(\alpha)}$ covering a large ball $B_{R}$;

(3) Local gauges given on concentric balls $B_{R_{n}}$ covering $X=B_{R}$ or $\mathbb{R}^{d}$.

In all three scenarios, the patching procedure depends only on the trivial topology and differentiable structure of the base.

Scenario (1): Large cubes covered by smaller cubes. We first consider a covering consisting of (round) cubes, which admits simple intersection properties.

Let $Q_{R}$ be a smooth domain in $\mathbb{R}^{d}$, and consider a covering $\left\{Q_{\alpha}\right\}_{\alpha \in \Gamma}$ of $Q_{R}$ by smooth domains $Q_{\alpha}$ indexed by a subset $\Gamma$ of the lattice $\mathbb{Z}^{d}$. We equip $\mathbb{Z}^{d}$ with two norms: $|\alpha|_{\infty}=$ $\sup _{k}\left|\alpha_{k}\right|$ and $|\alpha|_{1}=\left(\sum_{k}\left|\alpha_{k}\right|^{2}\right)^{1 / 2}$. We say that two indices are adjacent if $\left|\alpha-\alpha^{\prime}\right|_{\infty} \leq 1$. If $\left|\alpha-\alpha^{\prime}\right|_{1} \leq 1$, we say that $\alpha$ and $\alpha^{\prime}$ are face-adjacent; if $\left|\alpha-\alpha^{\prime}\right|_{\infty}=1$ but $\left|\alpha-\alpha^{\prime}\right|_{1}>1$, then we say that $\alpha$ and $\alpha^{\prime}$ are corner-adjacent. We say that the covering $\left\{Q_{\alpha}\right\}_{\alpha \in \Gamma}$ is $\operatorname{good}$ if the following properties hold:

(a) The index set $\Gamma$ is of the form $\Gamma=\left\{\alpha \in \mathbb{Z}^{d}:|\alpha|_{\infty}<R_{\Gamma}\right\}$ for some $R_{\Gamma}>0$. 
(b) For each $\alpha$, there exist a sequence of shrinking domains $Q_{\alpha}=Q_{\alpha}^{(0)} \supset Q_{\alpha}^{(1)} \supset \cdots$, such that, for each $n \geq 0$,

$$
Q_{R} \subseteq \bigcup_{\alpha \in \Gamma} Q_{\alpha}^{(n)}, \quad \overline{Q_{\alpha}^{(n+1)}} \cap Q_{R} \subseteq Q_{\alpha}^{(n)} \cap Q_{R}
$$

(c) Two domains $Q_{\alpha}^{(n)}$ and $Q_{\alpha^{\prime}}^{\left(n^{\prime}\right)}$ intersect if and only if their indices are adjacent.

(d) Consider any $\alpha \in \Gamma$ and a subfamily $\Gamma^{\prime} \subseteq \Gamma$ of adjacent indices with the property that (i) the face-adjacent indices in $\Gamma^{\prime}$ are adjacent to each other and (ii) each corner-adjacent index in $\Gamma^{\prime}$ is adjacent to some face-adjacent index in $\Gamma^{\prime}$. Then for each $n \geq 1$ there exists a diffeomorphism $\Phi_{\Gamma^{\prime}}^{(n)}$ from $Q_{\alpha}^{(n)}$ into $\tilde{F}_{\Gamma^{\prime}}^{(n)}=\left(\cup_{\alpha^{\prime} \in \Gamma^{\prime}} Q_{\alpha^{\prime}}^{(n-1)}\right) \cap Q_{\alpha}^{(n)}$, which equals the identity in $F_{\Gamma^{\prime}}^{(n)}=\left(\cup_{\alpha^{\prime} \in \Gamma^{\prime}} Q_{\alpha^{\prime}}^{(n)}\right) \cap Q_{\alpha}^{(n)}$.

Given any cube $Q_{R}$ of sidelength $R>1$, we construct a good covering of $Q_{R}$ by round cubes (i.e., with rounded edges, so that they are smooth) with roughly unit sidelength (more precisely, between $1 / 2$ and 4 ) as follows. Rescaling by a factor $\simeq 1$ (say between $1 / 2$ and 2), we may assume that $R$ is an integer. Partition $Q_{R}$ into unit cubes $\tilde{Q}_{\alpha}$ with integer vertices, indexed in an obvious manner by $\Gamma \subseteq \mathbb{Z}^{d}$ as in (a). Rounding off the edges (uniformly in $\alpha$ ), we may replace each $\tilde{Q}_{\alpha}$ by a round cube, such that $\left\{1.1 \tilde{Q}_{\alpha}\right\}$ still covers $Q_{R}$. Fix a sequence $2>\lambda^{(0)}>\lambda^{(1)}>\cdots>1.1$, and define $Q_{\alpha}^{(n)}$ to be the enlargement $\lambda^{(n)} \tilde{Q}_{\alpha}$. It is then straightforward to verify that (b)-(d) hold for $\left\{Q_{\alpha}^{(n)}\right\}$.

Remark 3.6. We make the simple but crucial observation that the preceding construction of a good covering may be fixed depending only on the size $R$ of the large cube. Also, $Q_{R}$ may be taken to be a round cube as well; it does not affect the properties (a)-(d), as long as the edges are rounded off at a scale much smaller than 1.

Let $\left\{Q_{\alpha}\right\}_{\alpha \in \Gamma}$ be a good covering of $Q_{R}$, and suppose that a local data set $\left\{Q_{\alpha}, O_{(\alpha \beta)}\right\}$ for a G-bundle (with arbitrary regularity) is given. Our goal is to patch the local gauges up to form a global gauge on $Q_{R}$. More concretely, we find a gauge transformation $P_{(\alpha)}$ on each $Q_{(\alpha)}^{(N)}$, where $N=\# \Gamma$, such that

$$
P_{(\beta)}=P_{(\alpha)} \cdot O_{(\alpha \beta)} \quad \text { in } Q_{\alpha}^{(N)} \cap Q_{\beta}^{(N)} .
$$

To start the construction, we endow $\Gamma$ with the lexicographic ordering (i.e., $\alpha<\alpha^{\prime}$ if $\alpha_{i}<\alpha_{i}^{\prime}$, where $i$ is the first index where the components differ); we denote by $[\alpha]$ the ordinality of $\alpha$ in this covering (thus $1 \leq[\alpha] \leq N$ ). The simple key observation is that such an ordering insures that each $\alpha$ and $\Gamma^{\prime}=\left\{\alpha^{\prime}: \alpha^{\prime}\right.$ is adjacent to $\left.\alpha, \alpha^{\prime}<\alpha\right\}$ satisfy the condition of $(d)$.

We proceed inductively on $[\alpha]$, and construct $P_{(\alpha)}$ on $Q_{\alpha}^{([\alpha])}$ such that

$$
P_{(\beta)}=P_{(\alpha)} \cdot O_{(\alpha \beta)} \quad \text { in } Q_{\alpha}^{([\beta])} \cap Q_{\beta}^{([\beta])} \text {, for } \alpha \leq \beta .
$$

For the first element $[\alpha]=1$, we simply take $P_{(\alpha)}=I d$ on $Q_{\alpha}^{(1)}$. Now assume that $P_{\left(\alpha^{\prime}\right)}$ has been constructed on $Q_{\alpha^{\prime}}^{\left(\left[\alpha^{\prime}\right]\right)}$ for $\alpha^{\prime}<\alpha$, where $[\alpha]=n>1$. Define $\tilde{P}_{(\alpha)}$ in $\tilde{F}_{\left\{\alpha^{\prime}<\alpha\right\}}^{(n)}=$ $\left(\cup_{\alpha^{\prime}<\alpha} Q_{\alpha^{\prime}}^{(n-1)}\right) \cap Q_{\alpha}^{(n)}$ by

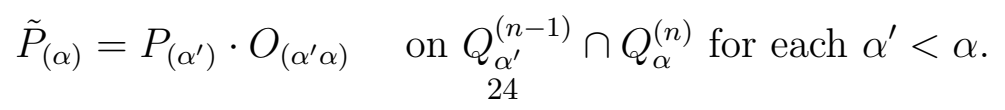


By construction, these expressions match on the intersections. Applying (d) in the definition of a good covering, we find a diffeomorphism $\Phi_{\left\{\alpha^{\prime}<\alpha\right\}}^{(n)}$ from $Q_{\alpha}^{(n)}$ into $\tilde{F}_{\left\{\alpha^{\prime}<\alpha\right\}}^{(n)}$, which equals the identity in $F_{\left\{\alpha^{\prime}<\alpha\right\}}^{(n)}=\left(\cup_{\alpha^{\prime}<\alpha} Q_{\alpha^{\prime}}^{(n)}\right) \cap Q_{\alpha}^{(n)}$. We simply define $P_{(\alpha)}$ in $Q_{\alpha}^{(n)}$ by the pullback

$$
P_{(\alpha)}=\tilde{P}_{(\alpha)} \circ \Phi_{\left\{\alpha^{\prime}<\alpha\right\}}^{(n)} .
$$

Next, suppose that local data for a connection $\left\{A_{(\alpha)}\right\}$ are also given. Then the gauge potential $A$ in the global gauge constructed above is described in terms of $A_{(\alpha)}$ and $P_{(\alpha)}$ as follows: Given a partition of unity $\chi_{(\alpha)}$ subordinate to $\left\{Q_{(\alpha)}^{(N)}\right\}$, we have

$$
A=\sum \chi_{(\alpha)}\left(A d\left(P_{(\alpha)}\right) A_{(\alpha)}-P_{(\alpha) ; x}\right) .
$$

The advantage of this patching procedure is that it relies only on the properties (a)-(d) of the good covering $\left\{Q_{\alpha}\right\}_{\alpha \in \Gamma}$, and is universal in the data $\left\{O_{(\alpha \beta)}\right\}$ or $\left\{A_{(\alpha)}\right\}$. Moreover, it is straightforward to infer properties of $P_{(\alpha)}$ and $A$ from those of $\left\{O_{(\alpha \beta)}\right\}$ and $\left\{A_{(\alpha)}\right\}$. Indeed, in the above construction, observe that $\left\{P_{(\alpha)}\right\}$ is constructed from $\left\{O_{(\alpha \beta)}\right\}$ using only the operations of (i) pointwise multplication, (ii) pullback by a diffeomorphism, (iii) restriction to a smooth subdomain and (iv) patching up local expressions which are consistent on the intersections. Any property of $\left\{O_{(\alpha \beta)}\right\}$ invariant under these operations transfers to $P_{(\alpha)}$. In particular, for any $k \geq 1$ and $p \geq \frac{d}{k}$,

$$
O_{(\alpha \beta)} \in \mathcal{G}_{l o c}^{k, p}\left(Q_{\alpha} \cap Q_{\beta}\right) \forall \alpha, \beta \Rightarrow P_{(\alpha)} \in \mathcal{G}_{l o c}^{k, p}\left(Q_{\alpha}^{(N)}\right) \forall \alpha .
$$

Regarding bounds for $A$, it is useful to introduce the following definition:

Definition 3.7. We say that a norm $Y$ on $\mathbb{R}^{d}$ is (patching-)admissible if:

- $Y$ is invariant under pullback by any diffeomorphism;

- $Y$ is invariant under any smooth cutoff;

- If $A \in Y$ and $O_{; x} \in Y$, then $A d(O) A \in Y$ with $\|A d(O) A\|_{Y} \lesssim_{\|A\|_{Y},\left\|O_{; x}\right\|_{Y}} 1$.

From the preceding observation regarding the construction of $P_{(\alpha)}$, as well as the explicit formula (3.3), we see that:

$$
O_{(\alpha \beta) ; x} \in Y\left(Q_{\alpha} \cap Q_{\beta}\right) \forall \alpha, \beta \text { and } A_{(\alpha)} \in Y\left(Q_{\alpha}\right) \forall \alpha \Rightarrow\|A\|_{Y\left(Q_{R}\right)} \lesssim 1,
$$

where the implicit constant depends only on the good covering (which, in turn, may be fixed depending only on $R$; cf. Remark (3.6)), $\sup _{\alpha}\left\|A_{(\alpha)}\right\|_{Y\left(Q_{\alpha}\right)}$ and $\sup _{\alpha, \beta}\left\|O_{(\alpha \beta)}\right\|_{Y\left(Q_{\alpha} \cap Q_{\beta}\right)}$.

Scenario (2): Large ball covered by small balls. Here, we wish to patch up local data for a Gbundle and a connection given on small balls centered inside $B_{R}$; this is the case we encounter in our applications. The idea is to reduce to Scenario (1) by a suitable diffeomorphism.

Consider a covering $\left\{B_{\alpha} \cap B_{R}\right\}$ of $B_{R}$ by finitely many balls. Let $\Phi$ be a bi-Lipschitz isomorphism from the cube $Q_{\lambda_{0} R}$ to $B_{R}$, where $\lambda_{0} \in(0, \infty)$ is to be fixed below. Let $\left\{Q_{\alpha}\right\}_{\alpha \in \Gamma}$ be a good covering of $Q_{\lambda_{0} R}$ as in Scenario (1). We wish to insure that the image of each $Q_{\alpha}$ under $\Phi_{R}$ is contained in a unit ball. Indeed, observe that, by scaling-invariance, the Lipschitz constant of $\Phi_{R}$ is independent of $R$, but decreases in $\lambda_{0}$. Hence, for any $\delta>0$, by choosing $\lambda_{0}$ sufficiently large (independent of $R$ ) we may insure that

$$
\Phi_{R}\left(Q_{\alpha}\right) \subseteq B_{\delta}(x) \quad \text { for some } x \in B_{R} .
$$


By Lebesgue's covering lemma, this ensures that $\Phi\left(Q_{\alpha}\right)$ is contained in some ball $B_{\alpha}$ in the covering. Finally, by rounding off the edges of $Q_{\lambda_{0} R}$, we may replace $Q_{\lambda_{0} R}$ by a round cube, and $\Phi$ by a diffeomorphism with uniform bounds. Note that this can be done while not disturbing the Lipschitz constant much (and thus (3.4) still holds), while the uniform bounds of higher derivatives would depend on $R$.

Remark 3.8. In the above procedure, note that $\lambda_{0}$ depends only on Lebesgue constant $\delta>0$ of the covering $\left\{B_{\alpha}\right\}$. In particular, if $B_{\alpha}$ 's are unit balls which are uniformly separated, so that the Lebesgue constant is $\simeq 1, \lambda_{0}$ may be fixed independent of $R$. The remaining components of the construction may be fixed depending only on the radius $R$ (recall also Remark 3.6).

We now apply the patching procedure in Scenario (1) to the pulled-back data $\left\{Q_{\alpha}, O_{(\alpha \beta)} \circ\right.$ $\left.\Phi, \Phi^{*} A_{(\alpha)}\right\}$, which are well-defined since each $\Phi\left(Q_{\alpha}\right)$ is contained in some ball $B_{\alpha}$ in the covering. Then we return to $B_{R}$ via $\Phi^{-1}$. As a result, we obtain a refinement $\left\{B_{\alpha}^{\prime}=\Phi\left(Q_{\alpha}^{(N)}\right)\right\}$ of the covering $\left\{B_{\alpha}\right\}$ (the index sets are different, but we abuse the notation and denote both by $\alpha$ ), as well as a gauge transform $P_{\alpha^{\prime}}$ in each $B_{\alpha^{\prime}}^{\prime}$, such that

$$
P_{(\alpha)}=P_{\left(\alpha^{\prime}\right)} \cdot O_{\left(\alpha^{\prime} \alpha\right)} \quad \text { on } B_{\alpha^{\prime}}^{\prime} \cap B_{\alpha}^{\prime} .
$$

Moreover, given a partition of unity subordinate to $\left\{B_{\alpha}^{\prime}\right\}$, the global gauge potential $A$ takes the form

$$
A=\sum \chi_{(\alpha)}\left(A d\left(P_{(\alpha)} A_{(\alpha)}-P_{(\alpha) ; x}\right) .\right.
$$

Finally, we obtain the following result:

Proposition 3.9. Let $R \geq 1$, and consider a covering $\left\{B_{\alpha} \cap B_{R}\right\}$ of $B_{R}$ by uniformly separated unit balls $B_{\alpha}$ centered inside $B_{R}$. Any G-bundle with $O_{(\alpha \beta)} \in \mathcal{G}^{k, \frac{d}{k}}\left(B_{\alpha} \cap B_{\beta} \cap B_{R}\right)$ admits a global gauge. Moreover, given any local data $\left\{A_{(\alpha)}\right\}$ for a connection on this $\mathbf{G}$ bundle satisfying $A \in W^{k-1, \frac{d}{k}}\left(B_{\alpha} \cap B_{R}\right)$, the global gauge potential satisfies $A \in W^{k, \frac{d}{k}}\left(B_{R}\right)$. More precisely, if

$$
\sup _{\alpha}\left\|A_{(\alpha)}\right\|_{W^{k-1, \frac{d}{k}}\left(B_{\alpha} \cap B_{R}\right)} \leq M, \quad \sup _{\alpha, \beta}\left\|O_{(\alpha \beta) ; x}\right\|_{W^{k-1, \frac{d}{k}}\left(B_{\alpha} \cap B_{\beta} \cap B_{R}\right)} \leq M,
$$

for some $M>0$, then

$$
\|A\|_{W^{k-1, \frac{d}{k}\left(B_{R}\right)}} \lesssim_{R, M} 1 .
$$

Scenario (3): $X=B_{R}$ or $\mathbb{R}^{d}$ covered by concentric balls. Finally, we consider the case when local data for a G-bundle and a connection are given all concentric balls $\left\{B_{R_{n}}\right\}_{n=1,2, \ldots}$ with $R_{n} \nearrow R$ or $\infty$.

Add a smaller ball $B_{R_{0}} \subset B_{R_{1}}$ to the covering. For $n \geq 2$, let $\Phi_{n}$ be a diffeomorphism from $B_{R_{n}}$ into $B_{R_{n-1}}$, which equals the identity on $B_{R_{n-2}}$. Define $P_{(n)}$ on $B_{R_{n}}$ inductively by $P_{(1)}=i d$ and

$$
P_{(n)}=\left(P_{(n-1)} \cdot O_{((n-1) n)}\right) \circ \Phi_{n} .
$$

Then we restrict the data and $P_{(n)}$ on $B_{R_{n}}$ to $B_{R_{n-1}}$. It follows by construction that, for $n<m$,

$$
P_{(m)}=P_{(n)} \cdot O_{(n m)} \quad \text { in } B_{R_{n-1}} .
$$

Given some local data $\left\{A_{(n)}\right\}$ for a connection, the global gauge potential is given by

$$
A=A d\left(P_{(n)}\right) A_{(n)}-P_{26}(n) ; x \quad \text { in } B_{R_{n-1}} .
$$


These expressions are consistent in the intersection (i.e., the smaller ball). Again, observe that $P_{(n)}$ is constructed by the same operations (i)-(iv) as in Scenario (1).

As a consequence this patching procedure, as well as Proposition 3.9, we obtain the following soft result, which is a starting point for the good global gauge theorems.

Proposition 3.10. Any G-bundle with regularity $\mathcal{G}_{l o c}^{k, \frac{d}{k}}$ on $X=B_{R}$ or $\mathbb{R}^{d}$ admits a global gauge. Moreover, for any $\mathbf{D} \in \mathcal{A}_{l o c}^{k-1, \frac{d}{k}}(X)$ on this $\mathbf{G}$-bundle, the global gauge potential satisfies $A \in W_{l o c}^{k-1, \frac{d}{k}}(X)$.

Proof. Let $\left\{U_{\alpha}, O_{(\alpha \beta)}\right\}$ be the local data for a G-bundle with regularity $\mathcal{G}_{\text {loc }}^{k, \frac{d}{k}}$ on $X=B_{R}$ or $\mathbb{R}^{d}$, and consider a smaller ball $B_{R^{\prime}}$ such that $\overline{B_{R^{\prime}}} \subseteq X$. By Lebesgue's covering lemma, there exists a refinement of $U_{\alpha}$ by balls $\left\{B_{\delta}(x) \cap B_{R^{\prime}}\right\}_{x \in B_{R^{\prime}}}$ of the same radius $\delta>0$. By Proposition 3.9, we obtain a global gauge on $B_{R^{\prime}}$. Since $R^{\prime}$ is arbitrary, Scenario (3) applies to a sequence of global gauges on $B_{R^{\prime}}$ with $R^{\prime} \nearrow R$ or $\infty$, and we obtain a global gauge on $X$. Existence of a corresponding global gauge potential for any $\mathcal{A}_{l o c}^{k-1, \frac{d}{k}}(X)$ connection is a quick corollary.

3.3. Uhlenbeck lemmas and elliptic regularity. Thanks to Proposition 3.10, we know that any $\mathcal{A}_{l o c}^{1, \frac{d}{2}}(X)$ connection admits a global gauge potential in $W_{l o c}^{1, \frac{d}{2}}(X)$. This is a natural setting for Uhlenbeck's lemma, which finds good local gauges under a gauge-invariant smallness assumption. These good local gauges furnish another main ingredient of the proof of the good global gauge theorems.

We start with the case of a ball $B_{1}$.

Theorem 3.11 (Uhlenbeck's lemma on a ball). Consider $\mathbf{D} \in \mathcal{A}_{\text {loc }}^{1, \frac{d}{2}}\left(B_{1}\right)$ of the form $\mathbf{D}=$ $\mathrm{d}+A$ with $A \in W^{1, \frac{d}{2}}\left(B_{1} ; \mathfrak{g}\right)$, which satisfies

$$
\|F[A]\|_{L^{\frac{d}{2}\left(B_{1}\right)}}<\epsilon_{0} .
$$

(1) There exists $O \in \mathcal{G}^{2, \frac{d}{2}}\left(B_{1}\right)$, unique up to multiplication by a constant element of $\mathbf{G}$, such that $\tilde{A}=\operatorname{Ad}(O) A-O_{; x} \in W^{1, \frac{d}{2}}\left(B_{1} ; \mathfrak{g}\right)$ obeys

$$
\partial^{\ell} \tilde{A}_{\ell}=0 \text { in } B_{1}, \quad x^{\ell} \tilde{A}_{\ell}=0 \text { on } \partial B_{1}
$$

and

$$
\|\tilde{A}\|_{W^{1, \frac{d}{2}\left(B_{1}\right)}} \lesssim\|F[A]\|_{L^{\frac{d}{2}\left(B_{1}\right)}} .
$$

(2) Let $A^{n}$ be a sequence of connections such that $A^{n} \rightarrow A$ in $W^{1, \frac{d}{2}}\left(B_{1} ; g\right)$. Let $\left(\tilde{A}^{n}, O^{n}\right)$ be given by (1) from $A^{n}$. Then passing to a subsequence and suitably conjugating each $\left(\tilde{A}^{n}, O^{n}\right)$ with a constant gauge transformation, we have

$$
\tilde{A}^{n} \rightarrow \tilde{A} \text { in } W^{1, \frac{d}{2}}\left(B_{1}\right), \quad O^{n} \rightarrow O \text { in } W^{2, \frac{d}{2}}\left(B_{1}\right) .
$$

Proof. For a proof of the existence claim in (1), see [30, Theorem 1.3]. For uniqueness, observe that the gauge transformation $\tilde{O} \in \mathcal{G}^{2, \frac{d}{2}}\left(B_{1}\right)$ between the two possible $\tilde{A}$ and $\tilde{A}^{\prime}$ satisfies the a-priori bound $\left\|\tilde{O}_{; x}\right\|_{W^{1, \frac{d}{2}\left(B_{1}\right)}} \lesssim \epsilon_{0}$, and also solves the div-curl system

$$
\partial^{\ell} \tilde{O}_{; \ell}=\operatorname{Ad}(\tilde{O})\left[\tilde{O}_{; \ell},\left(\tilde{A}^{\prime}\right)^{\ell}\right], \quad \partial_{j} \tilde{O}_{; k}-\partial_{k} \tilde{O}_{; j}=-\left[\tilde{O}_{; j}, \tilde{O}_{; k}\right]
$$


with the boundary condition $x^{\ell} \tilde{O}_{; \ell}=0$ on $\partial B_{1}$. It follows that $\tilde{O}_{; x}=0$, i.e., $\tilde{O}$ is constant.

To prove (2), observe first that the $W^{2, \frac{d}{2}}\left(B_{1}\right)$ norm of $O^{n}$ is uniformly bounded, thanks to the formula $O_{; x}^{n}=A d\left(O^{n}\right) A^{n}-\tilde{A}^{n}$. Thus, after passing to a subsequence, $O^{n} \rightarrow O^{\prime}$ and $\tilde{A}^{n} \rightarrow \tilde{A}^{\prime}$ in $W^{2, \frac{d}{2}}\left(B_{1}\right)$ and $W^{1, \frac{d}{2}}\left(B_{1}\right)$, respectively. This weak convergence is enough to justify

$$
\tilde{A}^{\prime}=A d\left(O^{\prime}\right) A-O_{; x}^{\prime} \text { in } B_{1}, \quad \partial^{\ell} \tilde{A}_{\ell}=0 \text { in } B_{1}, \quad x^{\ell} \tilde{A}_{\ell}=0 \text { on } \partial B_{1} .
$$

Hence, by the uniqueness statement in $(1),\left(\tilde{A}^{\prime}, O^{\prime}\right)$ coincides with $\left(\tilde{A}, O^{\prime}\right)$ up to a constant gauge transformation $O_{0}$. Applying $O_{0}$ to the sequence $\left(\tilde{A}^{n}, O^{n}\right)$, we may insure that $O^{n} \rightarrow O$ and $\tilde{A}^{n} \rightarrow \tilde{A}$ in $W^{2, \frac{d}{2}}\left(B_{1}\right)$ and $W^{1, \frac{d}{2}}\left(B_{1}\right)$, respectively.

To upgrade the weak convergence to strong convergence, we use the div-curl system for $\tilde{A}$. First, by the strong $W^{1, \frac{d}{2}}$ convergence $A^{n} \rightarrow A$ and the weak $W^{2, \frac{d}{2}}$ convergence $O^{n} \rightarrow O$, it follows that

$$
F\left[\tilde{A}^{n}\right]=A d\left(O^{n}\right) F\left[A^{n}\right] \rightarrow A d(O) F[A]=F[\tilde{A}] \quad \text { in } L^{\frac{d}{2}}\left(B_{1}\right) .
$$

Then by the div-curl system

$$
\partial^{\ell} \tilde{A}_{\ell}=0, \quad \partial_{j} \tilde{A}_{k}-\partial_{k} \tilde{A}_{j}=F\left[\tilde{A}^{n}\right],
$$

the weak $W^{1, \frac{d}{2}}$ convergence $\tilde{A}^{n} \rightarrow \tilde{A}$ is improved to strong convergence. Finally, by the formula $O_{; x}=\operatorname{Ad}(O) A-\tilde{A}$, the weak $W^{2, \frac{d}{2}}$ convergence $O^{n} \rightarrow O$ is also improved to strong convergence.

Theorem 3.11 was extended in [31] to a "removal of singularity" result for connections defined only on a punctured ball. Let $B_{r}^{\prime}=\left\{x \in \mathbb{R}^{d}: 0<|x|<r\right\}$.

Theorem 3.12 (Uhlenbeck's lemma on a punctured ball). Consider $\mathbf{D} \in \mathcal{A}_{\text {loc }}^{1, \frac{d}{2}}\left(B_{1+\delta}^{\prime}\right)$ for some $\delta>0$, which admits a representative $\mathbf{D}=\mathrm{d}+A$ with $A \in W_{l o c}^{1, \frac{d}{2}}\left(B_{1+\delta}^{\prime} ; \mathfrak{g}\right)$ and satisfies

$$
\|F[A]\|_{L^{\frac{d}{2}\left(B_{1}^{\prime}\right)}} \leq \epsilon_{0}^{\prime} .
$$

Then there exists $O \in \mathcal{G}_{\text {loc }}^{2, \frac{d}{2}}\left(B_{1}^{\prime}\right)$ such that $\tilde{A}=A d(O) A-O_{; x}$ obeys

$$
\partial^{\ell} \tilde{A}_{\ell}=0 \quad \text { in } B_{1}^{\prime}
$$

and

$$
\|\tilde{A}\|_{W^{1, \frac{d}{2}\left(B_{1}^{\prime}\right)}} \lesssim\|F[A]\|_{L^{\frac{d}{2}\left(B_{1}^{\prime}\right)}} .
$$

As a consequence, we see that $\tilde{A}$ is the restriction of a $\mathcal{A}_{l o c}^{1, \frac{d}{2}}$ connection on the full ball $B_{1+\delta}$. For a proof, we refer the reader to [31].

If $F$ satisfies higher (covariant) regularity bounds, then so does $\tilde{A}$ in the above theorems. This statement is most naturally formulated as an elliptic regularity result for the nonlinear div-curl system satisfied by $\tilde{A}$ with $\partial^{\ell} \tilde{A}_{\ell}=0$. In what follows, we omit the tilde for simplicity, and we focus on quantitative bounds in scaling-invariant spaces.

We start with a simple interior regularity result.

Lemma 3.13. Let $A \in W^{1, \frac{d}{2}}(B)$ be a solution to the nonlinear div-curl system

$$
\begin{aligned}
\partial_{j} A_{k}-\partial_{k} A_{j} & =F_{j k}-\left[A_{j}, A_{k}\right], \\
\partial^{\ell} A_{\ell} & =0 .
\end{aligned}
$$


If $\mathbf{D}^{(m)} F \in L^{\frac{d}{m+2}}(B)$ with $\frac{d}{m+2}>1$, then $\partial^{(m+1)} A \in L^{\frac{d}{n+2}}(\lambda B)$ for any $0 \leq \lambda<1$, with a bound depending only on $m,\left\|\mathbf{D}^{(m)} F\right\|_{L^{\frac{d}{m+2}(B)}},\|A\|_{L^{d}(B)}$ and $\lambda$.

Proof. Since it is a straightforward interior elliptic regularity argument, we only sketch the proof. We proceed by a simple induction on $m$; the key point is that $\partial^{(m)} F_{j k}$ and $\partial^{(m)}\left[A_{j}, A_{k}\right]$ in $L^{\frac{d}{m+2}}$ are controlled by $\mathbf{D}^{(m)} F$ in $L^{\frac{d}{m+2}}$ and the inductive bounds for $\partial^{\left(m^{\prime}+1\right)} A$ in $L^{\frac{d}{m^{\prime}+2}}$ $\left(0 \leq m^{\prime} \leq m\right)$.

When Theorem 3.11 is applied to a unit ball $B_{1}(x)$ centered near the boundary $\partial B_{R}$ of a larger ball, it is of interest to control regularity of $A$ up to the boundary $\partial B_{R}$. For this purpose, consider normalized angular derivatives $\not \partial=\left\{\frac{1}{|x|}\left(x_{j} \partial_{k}-x_{k} \partial_{j}\right)\right\}$ about the origin (at which $B_{R}$ is centered), and the corresponding covariant angular derivatives $\not D=$ $\left\{\frac{1}{|x|}\left(x_{j} \mathbf{D}_{k}-x_{k} \mathbf{D}_{j}\right)\right\}$. In any unit ball away from the origin, we show that higher angular regularity of $F$ implies the corresponding regularity of $A$ in the Coulomb gauge.

Lemma 3.14. Let $B$ be a unit ball in $\mathbb{R}^{d}$ such that $B \cap B_{1}(0)=\emptyset$, and let $A \in W^{1, \frac{d}{2}}(B)$ be a solution to the nonlinear div-curl system (3.8). If $\mathbf{D}^{(m)} F \in L^{\frac{d}{m+2}}(B)$ with $\frac{d}{m+2}>1$, then $\partial \not^{(m)} A \in L^{\frac{d}{m+2}}(\lambda B)$ for any $0 \leq \lambda<1$, with a bound depending only on $m,\left\|\not \not^{(m)} F\right\|_{L^{\frac{d}{m+2}}(B)}$, $\|A\|_{W^{1, \frac{d}{2}(B)}}$ and $\lambda$.

Proof. This lemma is most simply proved by commuting with the Lie derivatives with respect to the normalized rotation vector fields $\bar{\Omega}_{j k}=\frac{1}{d(0, B)} \Omega_{j k}$; these are isometries and thus exactly commute with the div-curl system. Moreover, their lengths are comparable to 1 (independent of $B)$, so that $\left|\mathcal{L}_{\bar{\Omega}}^{(\leq n)} A\right| \simeq_{n}\left|\partial^{(\leq n)} A\right|$.

As before, when $p=\frac{d}{n+2}>1$, the statement follows (with explicit bounds) by an induction on $n$. By the trace theorem and the (angular) Sobolev inequality, observe that

$$
\|u\|_{L_{r}^{\infty} L_{\Theta}^{\frac{d-1}{m+1}(B)}} \lesssim\|u\|_{L^{\frac{d}{m+2}(B)}}+\|\partial u\|_{L^{\frac{d}{m+2}}(B)} .
$$

Using this inequality and Hölder, we may control $\mathcal{L}_{\bar{\Omega}}^{(n)} F$ and $\bar{\Omega}^{(n)}\left[A_{j}, A_{k}\right]$ in $L^{\frac{d}{n+2}}$ by $\not^{(\leq n)} F$ in $L^{\frac{d}{n+2}}$ and the inductive bounds for $\partial \not^{(\leq m)} A$ in $L^{\frac{d}{m+2}}$. Then we may proceed as in the proof of Lemma 3.13.

Remark 3.15. As in Theorem 3.11(2), an argument similar to Lemma 3.13 (resp. Lemma 3.14) for the div-curl system for $\tilde{A}$ leads to strong convergence of $\partial^{(\leq m+1)} \tilde{A}^{n}$ and $\partial^{(\leq m+2)} O^{n}$ in $L^{\frac{d}{n+2}}(\lambda B)$ (resp. $\partial^{(\leq m+1)} \tilde{A}^{n}$ and $\partial^{(\leq 2)} \not^{(\leq m)} O^{n}$ in $L^{\frac{d}{n+2}}\left(\lambda B \cap B_{R}\right)$ ), provided that $A^{n} \rightarrow A$ in $W^{m, \frac{d}{m+1}}$. We omit the straightforward proof.

Next, we record a simple interior regularity result for the div-curl system of $O$.

Lemma 3.16. Let $O \in W^{2, \frac{d}{2}}(B)$ be a solution to the div-curl system

$$
\begin{aligned}
\partial_{j} O_{; k}-\partial_{k} O_{; j} & =\left[O_{; j}, O_{; k}\right] \\
\partial^{\ell} O_{; \ell} & =H .
\end{aligned}
$$

If $H \in \ell^{1} L^{\frac{d}{2}}(B)$, then $O_{; x} \in \ell^{1} W^{1, \frac{d}{2}}(\lambda B)$ for any $0 \leq \lambda<1$, with the bound

$$
\left\|O_{; x}\right\|_{\ell^{1} \dot{W}^{1, \frac{d}{2}}(\lambda B)} \lesssim_{\lambda}\|H\|_{\ell^{1} L^{\frac{d}{2}(B)}}+\left\|O_{; x}\right\|_{W^{1, \frac{d}{2}}(B)}^{2} .
$$


Moreover, if $\left(O^{\prime}, H^{\prime}\right) \in W^{2, \frac{d}{2}}(B) \times \ell^{1} L^{\frac{d}{2}}(B)$ is another solution to (3.9), then $\left\|O_{; x}-O_{; x}^{\prime}\right\|_{\ell^{1} \dot{W}^{1, \frac{d}{2}}(\lambda B)} \lesssim_{\lambda}\left\|H-H^{\prime}\right\|_{\ell^{1} L^{\frac{d}{2}(B)}}+\left(\left\|O_{; x}\right\|_{W^{1, \frac{d}{2}}(B)}+\left\|O_{; x}^{\prime}\right\|_{W^{1, \frac{d}{2}}(B)}\right)\left\|O_{; x}-O_{; x}^{\prime}\right\|_{W^{1, \frac{d}{2}(B)}}$.

The key point is that $\left[O_{; j}, O_{; k}\right]$ in $\ell^{1} L^{\frac{d}{2}}(B)$ can be estimated by $O_{; j}, O_{; k}$ in $W^{1, \frac{d}{2}}(B)$. We omit the obvious proof.

The $\ell^{1} \dot{W}^{1, \frac{d}{2}}$ bound on $O_{; x}$ is useful as it implies continuity of $O$. More precisely, we have the following:

Lemma 3.17. If $O_{; x} \in \ell^{1} W^{1, \frac{d}{2}}(B)$, then $O$ is continuous on $B$.

Proof. Without loss of generality, let $x_{1}$ be farther away from $\partial B$ than $x_{2}$. As in the proof of Morrey's inequality, we have

$$
d\left(O\left(x_{1}\right), O\left(x_{2}\right)\right) \lesssim \int_{B\left(x_{1}, 2 r\right)} \frac{\left|O_{; x}\right|}{\left|x-x_{1}\right|^{d-1}}+\frac{\left|O_{; x}\right|}{\left|x-x_{2}\right|^{d-1}} \mathrm{~d} x .
$$

The last integral may be estimated in terms of the Besov norm of the extension of $O_{; x}$, and vanishes as $x_{1} \rightarrow x_{2}$.

3.4. Good global gauge theorem on the ball. The goal of this subsection is to prove Theorem 1.4. The overall proof is divided into two steps:

- First, we prove the quantitative statements under the assumption that $\mathbf{D}$ admits a global gauge potential $A \in \dot{W}^{1, \frac{d}{2}}\left(B_{R}\right)$.

- Next, using softer arguments, we remove the global gauge assumption.

In the first step, the idea is to produce local gauges on balls $B_{1}(x)$ centered inside $B_{R}$ using Uhlenbeck's lemma, and then patch them up to a global gauge on $B_{R}$. To handle balls near the boundary, the following simple extension procedure is helpful.

Lemma 3.18. Let $A \in W^{1, \frac{d}{2}}\left(B_{R}\right)$ with $A_{r}=0$ on $\partial B_{R}$. Extend $A$ outside $B_{R}$ by

$$
\bar{A}_{r}\left(\frac{R^{2}}{r}, \Theta\right)=-A_{r}(r, \Theta), \quad \bar{A}_{\Theta}\left(\frac{R^{2}}{r}, \Theta\right)=A_{\Theta}(r, \Theta) .
$$

Then the extension obeys

$$
F[\bar{A}]\left(\frac{R^{2}}{r}, \Theta\right)=F[A](r, \Theta) \quad \text { for } r<R .
$$

The proof is an easy algebra computation, which we omit. We now carry out the first step.

Proposition 3.19. Theorem 1.4 holds under the additional assumption that $A \in \dot{W}^{1, \frac{d}{2}}\left(B_{R}\right)$.

Proof. By rescaling, we may set $r=1$, i.e., $\underline{r}_{c}^{\epsilon_{*}}[A] \geq 1$. Then we need to show that (1.6) holds with an implicit constant depending only on $\epsilon_{*}$ and $R$, provided that $\epsilon_{*}$ is sufficiently small compared to a universal constant.

If $R \lesssim 1$, then the conclusion of Theorem 1.4 follows by Uhlenbeck's lemma, so we may assume that $R>10$ (say). Applying Lemma 3.5, we may assume, without loss of generality that $A_{r}=0$. Then we extend $A$ outside $B_{R}$ via Lemma 3.18, By (3.10), it follows that the extended connection still has concentration radius $\gtrsim 1$ in $B_{R+10}$. Choosing $\epsilon_{*}$ sufficiently 
small, we may insure that Uhlenbeck's lemma applies to the extended connection on balls of radius 2 centered in $B_{R}$.

Consider a covering $\left\{B_{\alpha}\right\}$ of $B_{R}$ by uniformly separated unit balls centered in $B_{R}$, and apply Uhlenbeck's lemma on each $2 B_{\alpha}$ to obtain local data $A_{(\alpha)} \in W^{1, \frac{d}{2}}\left(2 B_{\alpha}\right)$ and $O_{(\alpha \beta)} \in$ $\mathcal{G}^{2, \frac{d}{2}}\left(2 B_{\alpha} \cap 2 B_{\beta}\right)$. By Lemma 3.13, we see that $A_{(\alpha)}$ enjoys higher regularity properties in each interior ball $B_{\alpha}$ (i.e., $2 B_{\alpha} \cap \partial B_{R}=\emptyset$ ). For a boundary ball $B_{\alpha}$, i.e., $2 B_{\alpha} \cap \partial B_{R} \neq \emptyset$, we first obtain higher angular regularity of $A$ in $B_{\alpha} \cap B_{R}$ by Lemma 3.14, and then also regularity in the radial direction by the equations

$$
\partial_{r} A_{r}=-\operatorname{div}_{\Theta} A_{\Theta}, \quad \partial_{r} A_{\Theta}=\partial_{\Theta} A_{r}+\left[A_{r}, A_{\Theta}\right]+F_{r \Theta},
$$

as well as radial covariant derivative bounds on $F_{r \Theta}$. Finally, observe that the desired higher regularity of $O_{(\alpha \beta)}$ in $B_{\alpha} \cap B_{\beta} \cap B_{R}$ follows from the equation $O_{(\alpha \beta) ; x}=\operatorname{Ad}\left(O_{(\alpha \beta)}\right) A_{(\beta)}-A_{(\alpha)}$ and the bounds for $A_{(\alpha)}, A_{(\beta)}$.

As a result, on the covering $\left\{B_{\alpha} \cap B_{R}\right\}$, we obtain local data $O_{(\alpha \beta)} \in W^{k, \frac{d}{k}}\left(B_{\alpha} \cap B_{\beta} \cap B_{R}\right)$ and $A_{(\alpha)} \in W^{k, \frac{d}{k}}\left(B_{(\alpha)} \cap B_{R}\right)$, provided that $\mathbf{D}^{(k)} F \in L^{\frac{d}{k}}$ (with $k \geq 1, \frac{d}{k}>1$ ). We are in a position to apply Proposition 3.9, from which the conclusion of Theorem 1.4 follows.

Finally, we remove the global gauge assumption, and thereby complete the proof of Theorem 1.4

Completion of proof of Theorem 1.4. Consider a sequence $R_{n} \nearrow R$. Apply Proposition 3.19 to each $A \uparrow_{B_{R_{n}}}$, which gives rise to $\tilde{A}^{(n)}$ and $O^{(n)}$ such that

$$
\begin{aligned}
O_{; j}^{(n)} & =\operatorname{Ad}\left(O^{(n)}\right) A_{j}-\tilde{A}_{j}^{(n)} \\
\partial_{k} O_{; j}^{(n)} & =\left[O_{; k}^{(n)}, \operatorname{Ad}\left(O^{(n)}\right) A_{j}\right]+\operatorname{Ad}\left(O^{(n)}\right) \partial_{k} A_{j}-\partial_{k} \tilde{A}_{j}^{(n)}
\end{aligned}
$$

It follows that $O_{; x}^{(m)}$ is uniformly bounded in $W^{1, \frac{d}{2}}$ on each fixed $B_{R^{\prime}}$. Therefore, after passing to a subsequence, there exists $O \in W_{l o c}^{2, \frac{d}{2}}\left(B_{R} ; \mathbb{R}^{N \times N}\right)$ such that $O^{(n)} \rightarrow O$ in $W^{2, \frac{d}{2}}\left(B_{R^{\prime}} ; \mathbb{R}^{N \times N}\right)$ for every $0<R^{\prime}<R$ and $O^{(n)} \rightarrow O$ a.e. on $B_{R}$. Hence, $O \in \mathcal{G}_{l o c}^{2, \frac{d}{2}}\left(B_{R}\right)$ and moreover

$$
\tilde{A}_{j}=A d(O) A_{j}-O_{; j}
$$

is the weak limit of $\tilde{A}^{(n)}$ in $W_{l o c}^{1, \frac{d}{2}}$. Since the $\dot{W}^{1, \frac{d}{2}}\left(B_{R_{n}}\right)$ norm of $\tilde{A}^{(n)}$ is uniformly bounded in $n$, it follows that $\|\tilde{A}\|_{\dot{W}^{1, \frac{d}{2}}\left(B_{R}\right)} \lesssim_{\|F\|_{L^{\frac{d}{2}}}} 1$.

3.5. Good global gauge theorem on the whole space. Next, we establish Theorem 1.5,

Proof of Theorem 1.5. By rescaling, we set $\underline{R}_{c}=1$. Throughout this proof, we work with global gauge potentials in $W_{l o c}^{1, \frac{d}{2}}\left(\mathbb{R}^{d}\right)$ for $\mathbf{D}$, which exists thanks to Proposition 3.10.

The first main task is to find a good gauge in a suitable exterior domain. By hypothesis, and our normalization $\underline{R}_{c}=1$, we have $\|F[A]\|_{L^{\frac{d}{2}\left(\mathbb{R}^{d} \backslash \bar{B}\right)}}<\epsilon_{*}$. Consider the inversion map

$$
\iota: x \mapsto y=\frac{x}{|x|^{2}},
$$


which clearly satisfies $\iota \circ \iota=i d$. Under $\iota$, the exterior region $\mathbb{R}^{d} \backslash \bar{B}$ is the image of the punctured unit ball $B^{\prime}$, and vice versa. The map $\iota$ is a conformal isometry, such that

$$
\left(\iota^{*} \delta\right)^{i j}=|x|^{4} \delta^{i j}, \quad \iota^{*}\left(\mathrm{~d} y^{1} \wedge \cdots \wedge \mathrm{d} y^{d}\right)=\frac{(-1)^{d}}{|x|^{2 d}} \mathrm{~d} x^{1} \wedge \cdots \wedge \mathrm{d} x^{d} .
$$

In particular, if $T$ is a covariant 2-tensor on $\iota(U) \subseteq \mathbb{R}^{d}$, then

$$
\int_{\iota(U)}\left(\sum_{i, j}\left|T_{y^{i} y^{j}}\right|^{2}\right)^{\frac{d}{4}}(y) \mathrm{d} y=\int_{U}\left(\sum_{i, j}\left|\iota^{*} T_{x^{i} x^{j}}\right|^{2}\right)^{\frac{d}{4}}(x) \mathrm{d} x .
$$

Choosing $\epsilon_{*}<\epsilon_{0}^{\prime}$, we have $\left\|\iota^{*} F\right\|_{L^{\frac{d}{2}\left(B^{\prime}\right)}}=\|F\|_{L^{\frac{d}{2}\left(\mathbb{R}^{d} \backslash \bar{B}\right.}}<\epsilon_{0}^{\prime}$, and we may apply Theorem 3.12 to find a local gauge in which the gauge potential satisfies $\tilde{A}_{(\infty)} \in \dot{W}^{1, \frac{d}{2}}(B)$. We define $A_{(\infty)}$ to be the local gauge potential of $\mathbf{D}=\iota^{*} \iota^{*} \mathbf{D}$ on $\mathbb{R}^{d} \backslash \bar{B}$ given by $A_{(\infty)}=\iota^{*} \tilde{A}_{(\infty)}$. Since $\partial\left(\iota^{*} \tilde{A}_{(\infty)}\right)=\iota^{*}\left(\partial \tilde{A}_{(\infty)}\right)$ and $\left\|\iota^{*} \partial \tilde{A}_{(\infty)}\right\|_{L^{\frac{d}{2}\left(\mathbb{R}^{d} \backslash \bar{B}\right)}}=\left\|\partial \tilde{A}_{(\infty)}\right\|_{L^{\frac{d}{2}\left(B^{\prime}\right)}}$, it follows that $A_{(\infty)} \in$ $\dot{W}^{1, \frac{d}{2}}\left(\mathbb{R}^{d} \backslash \bar{B}\right)$ and

$$
\left\|A_{(\infty)}\right\|_{L^{d} \cap \dot{W}^{1, \frac{d}{2}}\left(\mathbb{R}^{d} \backslash \bar{B}\right)} \lesssim \epsilon_{*}
$$

On the other hand, by Theorem 1.4 applied to $5 B$, we obtain a local gauge potential $A_{(0)} \in$ for such that

$$
\left\|A_{(0)}\right\|_{L^{d} \cap \dot{W}^{1, \frac{d}{2}}(5 B)} \lesssim_{\epsilon_{*}, \underline{r}_{c}^{-1}} 1 .
$$

By construction there exists $O \in \mathcal{G}_{\text {loc }}^{2, \frac{d}{2}}(5 B \backslash \bar{B})$ such that

$$
A_{(0)}=A d(O) A_{(\infty)}-O_{; x} \quad \text { in } 5 B \backslash \bar{B} .
$$

By this relation, (3.12) and (3.13), on $5 B \backslash \bar{B}$ we have

$$
\left\|O_{; x}\right\|_{L^{d} \cap \dot{W}^{1, \frac{d}{2}}(5 B \backslash \bar{B})} \lesssim_{\epsilon_{*}, \underline{r}_{c}^{-1}} 1 .
$$

Using the partial approximation lemma (Lemma 3.2) and performing 0-homogeneous extension outside a suitable sphere, it is straightforward to construct a gauge transform $\tilde{O}_{(\infty)}$ on $\mathbb{R}^{d} \backslash \bar{B}$ satisfying the following properties:

- $\tilde{O}_{(\infty)}=O$ in $2 B \backslash \bar{B}$

- $\tilde{O}_{(\infty)}(r \Theta)=\tilde{O}_{(\infty)}(4 \Theta)$ for $\Theta \in \mathbb{S}^{d-1}$ and $r \geq 4$

- $\left\|\tilde{O}_{(\infty)}\right\|_{L^{d} \cap \dot{W}^{1, \frac{d}{2}}(5 B \backslash \bar{B})} \lesssim_{\epsilon_{*}, \underline{r}_{c}^{-1}} 1$;

- $\tilde{O}_{(\infty)}$ is $C^{\infty}$ in $5 B \backslash \overline{3 B}$ with $\left\|O_{(\infty)}\right\|_{C^{N}(5 B \backslash \overline{3 B})} \lesssim_{\epsilon_{*}, \underline{r}_{c}^{-1}, N} 1$ for all $N \geq 0$.

Using $\tilde{O}_{(\infty)}$ to patch up the local gauges in $2 B$ and $\mathbb{R}^{d} \backslash \bar{B}$, we obtain the global gauge potential

$$
A_{x}=\left\{\begin{array}{cl}
A_{(0) x} & \text { on } 2 B \\
A d\left(\tilde{O}_{(\infty)}\right) A_{(\infty) x}-\tilde{O}_{(\infty) ; x} & \text { on } \mathbb{R}^{d} \backslash \bar{B}
\end{array}\right.
$$

Let $O_{(\infty)}$ be the smooth 0 -homogeneous map on $\mathbb{R}^{d} \backslash\{0\}$ defined by $O_{(\infty)}(r \Theta)=\tilde{O}_{(\infty)}(4 \Theta)$, and define $B_{x}=A_{x}+\chi O_{(\infty) ; x}$. By (3.12), (3.13) and the preceding bounds for $\tilde{O}_{(\infty)}$, the desired bounds (1.8) follow. 
3.6. Topological classes of rough connections. Here, we verify the results stated in Section 1.4. Our first goal is to prove homotopy equivalence of $O_{(\infty)}$ of different good representations of the same connection (Proposition 1.6). We need a few lemmas.

Lemma 3.20. Let $O \in \mathcal{G}^{2, \frac{d}{2}}(A)$, where $A=\left\{x \in \mathbb{R}^{d}: R_{1}<|x|<R_{2}\right\}$ is an annulus. For almost every $R \in\left(R_{1}, R_{2}\right), O \uparrow_{\partial B_{R}}$ is continuous, which are all homotopic to each other.

By this lemma, we may define $[O]$ to be the homotopy class (as continuous maps $\mathbb{S}^{d-1} \rightarrow \mathbf{G}$ ) of the restriction of $O$ to $\partial B_{R}$ for almost every $R$. We refer to such $R$ 's as generic radii.

Proof. Since the boundary of $A$ is smooth, we may approximate $O$ by $O^{n} \in C^{\infty}(A ; \mathbf{G})$ in the $W^{2, \frac{d}{2}}\left(A ; \mathbb{R}^{N \times N}\right)$-topology [26, 31]. After passing to a subsequence, for almost every $R \in\left(R_{1}, R_{2}\right)$, we have

$$
O^{n} \uparrow_{\partial B_{R}} \rightarrow O^{n} \uparrow_{\partial B_{R}} \text { in } W^{2, \frac{d}{2}}\left(\partial B_{R} ; \mathbb{R}^{N \times N}\right) .
$$

The lemma now follows from the observation that $W^{2, \frac{d}{2}}\left(\partial B_{R} ; \mathbb{R}^{N \times N}\right) \hookrightarrow C^{0}\left(\partial B_{R} ; \mathbb{R}^{N \times N}\right)$, due to the Sobolev embedding on spheres.

Lemma 3.21. Let $\delta>0$ and let $O \in \mathcal{G}^{2, \frac{d}{2}}(\tilde{A})$, where $\tilde{A}=\left\{x \in \mathbb{R}^{d}: R_{1}-\delta<|x|<R_{2}\right\}$. Then there exists an extension $\tilde{O} \in \mathcal{G}^{2, \frac{d}{2}}\left(B_{R_{2}}\right)$ such that $\tilde{O} \uparrow_{A}=O \uparrow_{A}$ if and only if $[O]=[i d]$.

In this lemma, $[O]$ is defined by viewing $O$ as defined on either the annulus $\tilde{A}$ or $A$; both give the same answer by Lemma 3.20. Our proof is qualitative, in that we make no claim regarding the size of $\tilde{O} \in \mathcal{G}^{2, \frac{d}{2}}\left(B_{R_{2}}\right)$.

Proof. We first prove the "only if" part. By Lemma 3.2 (with $V=\emptyset$ and $U=W=B_{R_{2}}$ ), there exists an approximating sequence $O^{n} \in C^{\infty}\left(B_{R_{2}} ; \mathbf{G}\right)$, which approaches $O$ in the $W^{2, \frac{d}{2}}\left(B_{R_{2}} ; \mathbb{R}^{N \times N}\right)$-topology. Recalling the proof of Lemma $[3.20$, we see that $[O]$ is the homotopy class of $O^{n} \uparrow_{\partial B_{R}}$ for any $\partial B_{R} \subseteq B_{R_{2}}$, provided that $n$ is sufficiently large. Now, the whole map $O^{n}: B_{R_{2}} \rightarrow \mathbf{G}$ provides a homotopy from $O^{n} \uparrow_{\partial B_{R_{2}}}$ to the constant map $O^{n} \uparrow_{\{0\}}$, which in turn is homotopic to the identity map.

Next, we prove the "if" part. First, by Lemma 3.2 , there exists $O^{\prime} \in \mathcal{G}^{2, \frac{d}{2}}\left(R_{1}-\frac{4}{3} \delta<\right.$ $\left.|x|<R_{2}\right)$ such that $O^{\prime} \in C^{\infty}\left(R_{1}-\frac{4}{3} \delta<|x|<R_{1}-\frac{1}{4} \delta ; \mathbf{G}\right), O^{\prime} \uparrow_{A}=O \uparrow_{A}$. By Lemma 3.20, $\left[O^{\prime}\right]=[O]=[i d]$. Working in the smooth category, we may find $\tilde{O} \in \mathcal{G}^{2, \frac{d}{2}}\left(B_{R_{2}}\right)$ such that $\tilde{O} \uparrow_{A}=O^{\prime} \uparrow_{A}$ while $\tilde{O} \in C^{\infty}\left(|x|<R_{2}-\frac{1}{2} \delta\right)$.

Lemma 3.22. Let $O \in \mathcal{G}^{2, \frac{d}{2}}\left(\mathbb{R}^{d} \backslash \bar{B}\right)$. Then $[O]=[i d]$.

In this lemma, $[O]$ is defined by viewing $O$ as defined on an annulus $A \subseteq \mathbb{R}^{d} \backslash B$.

Proof. Without loss of generality, let $U=\mathbb{R}^{d} \backslash \bar{B}_{1}$. We also observe that it suffices to prove $[O]=[$ const $]$. As before, by Lemma 3.2 (more precisely, a slight variant for the exterior domain) there exists an approximating sequence $O^{n} \in C^{\infty}(U ; \mathbf{G})$, which approaches $O$ in the $W^{2, \frac{d}{2}}\left(U ; \mathbb{R}^{N \times N}\right)$-topology, where $[O]$ is the homotopy class of $O^{n} \uparrow_{\partial B_{R}}$ for any $\partial B_{R} \subseteq U$, provided that $n$ is sufficiently large.

By Sobolev embedding, note that

$$
\int_{U}\left|O_{; x}^{n}\right|^{d} \mathrm{~d} x<\infty \quad \text { for all } n
$$


In the polar coordinates $(r, \Theta) \in(0, \infty) \times \mathbb{S}^{d-1}$, it follows that

$$
\int_{1}^{\infty} \int_{\mathbb{S}^{d-1}}\left|\partial_{\Theta} O^{n}(r, \Theta)\right|^{d} \mathrm{~d} V_{\mathbb{S}^{d-1}}(\Theta) \frac{\mathrm{d} r}{r}<\infty \quad \text { for all } n,
$$

which implies that $\liminf _{r \rightarrow \infty}\left\|\partial_{\Theta} O^{n}(r, \Theta)\right\|_{L^{d}\left(\mathbb{S}^{d}\right)}=0$. The desired conclusion $[O]=[$ const $]$ now follows.

We are ready to prove Proposition 1.6.

Proof of Proposition 1.6. By suitably replacing $\chi$, we may assume that $1-\chi$ vanishes outside the unit ball $B$.

Proof of (1). By equivalence of $\left(O_{(\infty)}, B_{x}\right)$ and $\left(O_{(\infty)}^{\prime}, B_{x}^{\prime}\right)$, there exists $O \in \mathcal{G}_{l o c}^{2, \frac{d}{2}}\left(\mathbb{R}^{d}\right)$ such that

$$
-O_{(\infty) ; x}+B_{x}=-A d(O) O_{(\infty) ; x}^{\prime}-O_{; x}+A d(O) B_{x}^{\prime} .
$$

From simple computation, it follows that

$$
\left(O_{(\infty)}^{-1} O O_{(\infty)}^{\prime}\right)_{; x}=A d\left(O_{(\infty)}^{-1} O\right) B_{x}^{\prime}-A d\left(O_{(\infty)}^{-1}\right) B_{x}
$$

which implies that $O_{(\infty)}^{-1} O O_{(\infty)}^{\prime} \in \mathcal{G}^{1, d}\left(\mathbb{R}^{d} \backslash \bar{B}\right)$. Applying Lemmas 3.21 and 3.22 to $O$ and $O_{(\infty)}^{-1} O O_{(\infty)}^{\prime}$, respectively, it follows that

$$
[i d]=[O]=\left[O_{(\infty)}^{-1} O O_{(\infty)}^{\prime}\right]
$$

Therefore, $\left[O_{(\infty)}\right]=\left[O_{(\infty)}^{\prime}\right]$, as desired.

Proof of (2). Since $\left[O_{(\infty)}^{\prime} O_{(\infty)}^{-1}\right]=[i d]$, by Lemma 3.21 there exists a gauge transform $P \in \mathcal{G}^{2, \frac{d}{2}}(2 B)$ such that $P=O_{(\infty)}^{\prime} O_{(\infty)}^{-1}$ in $2 B \backslash \bar{B}$. Extend $P$ as a 0 -homogeneous map outside $2 B$; we abuse the notation and refer to the extension again by $P$ (thus, $P=O_{(\infty)}^{\prime} O_{(\infty)}^{-1}$ in $\left.\mathbb{R}^{d} \backslash \bar{B}\right)$. Apply the gauge transform $P$ to $A_{x}=-\chi O_{(\infty) ; x}+B_{x}$, and define $B_{x}^{\prime}$ by the decomposition $A d(P) A_{x}-P_{; x}=-\chi O_{(\infty) ; x}^{\prime}+B_{x}^{\prime}$. From $P \in \mathcal{G}^{2, \frac{d}{2}}(2 B)$, it follows that $B_{x}^{\prime} \in L^{d} \cap \dot{W}^{1, \frac{d}{2}}(2 B)$. Moreover, outside $2 B$,

$$
B_{x}^{\prime}=A d(P) B_{x}
$$

Observe that 0-homogeneity of $P$ is sufficient to ensure $A d(P) B_{x} \in L^{d} \cap \dot{W}^{1, \frac{d}{2}}\left(\mathbb{R}^{d} \backslash \overline{2 B}\right)$. Hence $\left(O_{(\infty) ; x}^{\prime}, B_{x}^{\prime}\right)$ is also a good representation, as desired.

Finally, we prove Proposition 1.9 .

Proof of Proposition 1.9. By scaling, we may set $R=1$. Arguing as in the proof of Theorem 1.5, we find local gauge potentials $A_{(\infty)}$ and $A_{(\infty)}^{\prime}$ in $\mathbb{R}^{d} \backslash \bar{B}$ satisfying (3.12). By construction, there exist $O, O^{\prime} \in \mathcal{G}^{2, \frac{d}{2}}(5 B \backslash \bar{B})$ such that

$$
A=A d(O) A_{(\infty)}-O_{; x}, \quad A^{\prime}=A d\left(O^{\prime}\right) A_{(\infty)}^{\prime}-O_{; x}^{\prime} \quad \text { in } 5 B \backslash \bar{B} .
$$

From the proof of Theorem 1.5, as well as Definition 1.8, note that the topological classes $[A]$ and $\left[A^{\prime}\right]$ are determined by the homotopy classes $[O]$ and $\left[O^{\prime}\right]$, respectively, as defined in Lemma 3.20. In particular, it suffices to prove that $O \uparrow \partial B_{r}$ and $O^{\prime} \uparrow \partial B_{r}$ are homotopic to each other for a generic $1<r<5$, in the sense of Lemma 3.20. 
Since $\left.\left\|A-A^{\prime}\right\|_{L^{d}(5 B}\right) \leq \epsilon_{*}$, the difference $O_{; x}-O_{; x}^{\prime}$ obeys the bound

$$
\left\|O_{; x}-O_{; x}^{\prime}\right\|_{L^{d}(5 B \backslash \bar{B})} \lesssim \epsilon_{*},
$$

which holds independently of possible additional constant gauge transformations for $O$ or $O^{\prime}$. By the pigeonhole principle, the following bound holds some generic $1<r<5$ :

$$
\left\|O_{; x}-O_{; x}^{\prime}\right\|_{L^{d}\left(\partial B_{r}\right)} \lesssim \epsilon_{*} .
$$

After a suitable constant gauge transformation (which does not change the homotopy class), it follows that $O$ and $O^{\prime}$ are close in $C^{\frac{1}{d}}\left(\partial B_{r}\right)$, and therefore belong to the same homotopy class.

\section{Excision, Gluing AND EXtension of YANG-Mills initial DATA SETS}

In this section, we provide proofs of the results stated in Section 1.6 concerning the YangMills initial data sets.

4.1. Solvability results for the inhomogeneous Gauss equation. In this subsection, we address the question of solvability for divergence equations

$$
\left(\mathbf{D}^{(a)}\right)^{\ell} e_{\ell}=h
$$

in exterior of a convex domain.

To quantify the constants, we need to quantify the geometry of a convex domain. Let $K$ be a convex domain with barycenter $x_{K}$. By convexity, for each $\Theta \in \mathbb{S}^{d-1}$, there exists a unique intersection $f_{K}(\Theta)$ of $\partial K$ and the ray in the direction $\Theta$ emanating from $x_{K}$. Define the radius of $K$ by $R(K)=\sup _{x, y \in K}|x-y|$, and the Lipschitz constant of $K$ by

$$
L(K)=\sup _{\Theta, \Theta^{\prime} \in \mathbb{S}^{d-1}} \frac{\left|f_{K}(\Theta)-f_{K}\left(\Theta^{\prime}\right)\right|}{R(K)\left|\Theta-\Theta^{\prime}\right|} .
$$

Clearly, $R(K)$ is 1-homogeneous and $L(K)$ is scaling-invariant, in the sense that $R(\lambda K)=$ $\lambda R(K)$ and $L(\lambda K)=L(K)$ for $\lambda>0$.

We begin with a general solvability result for the usual divergence equation (i.e., $a=0)$.

Proposition 4.1. For any convex domain $K$, there exists a solution operator $T_{0}$ for the equation $\partial^{\ell} e_{\ell}=h$ with the following properties:

(1) (Boundedness) For $1<p<\infty$ and $1-\frac{d}{p}<\sigma<1+\frac{d}{p}$,

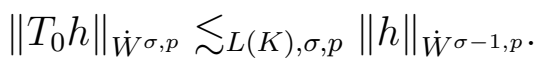

(2) (Exterior support) If $h=0$ in $\lambda K$, then $T_{0} h=0$ in $\lambda K$.

(3) (Higher regularity) If $h$ is smooth, so is $T_{0} h$.

Proof. In the case $K$ is a ball, this was considered in our prior work [19], where $T_{0}$ is constructed as a pseudodifferential operator of order -1 . Here we will use a slightly different but closely related solution operator.

First, we claim that given a unit vector $\omega \in \mathbb{S}^{d-1}$, we can construct an exact solution operator $T_{\omega}$ with smooth homogeneous symbol of order 1, and kernel supported in a small 
conic neighborhood of $\omega$. Our starting point is the simple observation that the following operator solves the divergence equation (say for $h \in C_{c}^{\infty}\left(\mathbb{R}^{d}\right)$ ):

$$
\tilde{T}_{e_{1}} h(x)=\int_{-\infty}^{x^{1}} e_{1} h\left(y^{1}, x^{2}, \ldots, x^{d}\right) \mathrm{d} y^{1},
$$

where $e_{1}$ is the unit vector $(1,0, \ldots, 0)$. This operator is translation-invariant with kernel

$$
e_{1} 1_{(0, \infty)}\left(x^{1}\right) \delta_{0}\left(x^{2}\right) \cdots \delta_{0}\left(x^{d}\right)
$$

which is supported on the ray $\left\{r e_{1}: r>0\right\}$. By rotation, for any unit vector $\omega \in \mathbb{S}^{d-1}$, we obtain an analogous translation-invariant solution operator $\tilde{T}_{\omega}$ whose kernel is supported on the ray $\{r \omega: r>0\}$. Moreover, given a smooth function $\tilde{\chi}_{\omega}\left(\omega^{\prime}\right)$ on $\mathbb{S}^{d-1}$ supported on a neighborhood $\hat{C}_{\omega} \subseteq \mathbb{S}^{d-1}$, the smooth average

$$
T_{\omega} h=\int \tilde{T}_{\omega^{\prime}}(h) \tilde{\chi}_{\omega}\left(\omega^{\prime}\right) \mathrm{d} \omega^{\prime}
$$

is a translation-invariant solution operator, whose kernel is smooth outside the origin, homogeneous of degree $-d+1$ and supported in the conic neighborhood $C_{\omega}=\left\{x \in \mathbb{R}^{d}: \frac{x}{|x|} \in \hat{C}_{\omega}\right\}$, as desired.

We now turn to the issue of insuring the exterior support property. If one were to work with the operators $\tilde{T}_{\omega}$, then it is easy to produce such an solution operator $T$ : We simply decompose the input into each angle $\omega$ and apply $\tilde{T}_{\omega}$, i.e., $T=\int_{\mathbb{S}^{d-1}} \tilde{T}_{\omega} \delta_{\omega}\left(\omega^{\prime}\right) \mathrm{d} \omega^{\prime}$. Then (formally) the exterior support property holds for any convex set $K$.

To use the operators $T_{\omega}$ with "fattened" kernel, we use a uniform conical partition of unity in the physical space $1=\sum \chi_{\omega}$ (centered at the origin) and define our solution operator $T_{0}$ to be

$$
T_{0}=\sum T_{\omega} \chi_{\omega}
$$

Making the angular support of each $\chi_{\omega}$ sufficiently narrow (which, of course, increases the number of partitions) depending on $L(K)$, we may insure the exterior support property of $T$.

Multiplication by each $\chi_{\omega}$ is bounded on $\dot{W}^{\sigma-1, p}$ thanks to Hardy's inequality, which holds since $|\sigma-1|<\frac{d}{p}$; hence (4.3) follows. The higher regularity property follows by differentiation.

Next, we generalize Proposition 4.1 to the inhomogeneous covariant Gauss equation (4.1) when $\|a\|_{\dot{H} \frac{d-2}{2}}$ is small by a perturbative argument.

Proposition 4.2. Let $\mathbf{D}=\mathrm{d}+a \in \mathcal{A}^{\frac{d-2}{2}, 2}\left(\mathbb{R}^{d}\right)$ satisfy $\|a\|_{\dot{H}} \frac{d-2}{2} \leq \epsilon_{*}$. For any convex domain $K$, there exists a solution operator $T_{a}$ for the equation $\mathbf{D}^{\ell} e_{\ell}=h$ with the following properties:

(1) (Boundedness) For $2 \leq p<\infty$ and $1-\frac{d}{p}<\sigma<\frac{d}{2}$,

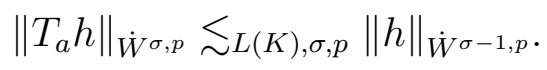

(2) (Exterior support) If $h=0$ in $\lambda K$, then $T_{a} h=0$ in $\lambda K$.

(3) (Higher regularity) If $a$ and $h$ is smooth, so is $T_{a} h$. 
Proof. We proceed in two steps.

Step 1: Definition of $T_{a}$. To define $T_{a}$, we solve the fixed point problem is

$$
e=T\left(h-\left[a^{\ell}, e_{\ell}\right]\right) \text {. }
$$

Let us abbreviate $\left[a^{\ell}, e_{\ell}\right]=a d(a) e$. Under the conditions for $p$ and $\sigma$, multiplication by $a$ takes $\dot{W}^{\sigma, p}$ into $\dot{W}^{\sigma-1, p}$ (this may be proved by the usual Littlewood-Paley trichotomy), so that we can estimate

$$
\|\operatorname{Tad}(a)\|_{\dot{W}^{\sigma, p} \rightarrow \dot{W}^{\sigma, p}} \lesssim\|a\|_{\dot{H}^{\frac{d-2}{2}}} .
$$

Therefore, for $\|a\|_{\dot{H}^{\frac{d-2}{2}}}$ sufficiently small, we find $T_{a}$ which clearly satisfies the boundedness and exterior support properties.

Step 2: Higher regularity. Here we assume that $\partial^{(m)} a \in \dot{H}^{\frac{d-2}{2}}$ and $\partial^{(m)} h \in \dot{W}^{\sigma-1, p}$ for $0 \leq m \leq n$, then we prove that $\partial^{(n)} e \in \dot{W}^{\sigma, p}$. We consider the case $n=1$; higher values of $n$ are dealt with in a similar manner. Differentiating our fixed point problem we get

$$
\partial e=T\left(\partial h-\left[a^{\ell}, \partial e_{\ell}\right]\right)-T\left(\left[\partial a^{\ell}, e_{\ell}\right]\right)+[\partial, T]\left(h-\left[a^{\ell}, e_{\ell}\right]\right)
$$

where we can estimate

$$
\|T([\partial a, e])+[T, \partial](h-[a, e])\|_{\dot{W}^{\sigma, p}} \lesssim\|e\|_{\dot{W}^{\sigma, p}}+\|h\|_{\dot{W}^{\sigma, p}}
$$

with an implicit constant depending on the $\dot{H}^{\frac{d-2}{2}}$ norms of $\partial a$ and $a$. Then we have a fixed point problem for $\partial e$, which is solved in $\dot{W}^{\sigma, p}$ to obtain the bound

$$
\|\partial e\|_{\dot{W}^{\sigma, p}} \lesssim\|h\|_{\dot{W}^{\sigma, p} \cap \dot{W}^{\sigma-1, p}}
$$

One minor issue here is that we do not a-priori know that $\partial e \in \dot{W}^{\sigma, p}$. But this can be easily circumvented by replacing the gradient with the appropriated divided difference.

Finally, we prove Theorem 1.14, where the smallness assumption for $a$ is removed. For simplicity, we restrict to the critical space $h \in \dot{H}^{\frac{d-6}{2}}$ where $d \geq 4$, which suffices for our main applications.

Proof of Theorem 1.14. We work from the case when $h$ is not differentiated (i.e., $h \in L^{\frac{d}{2}}$ ), and gradually move up to higher regularity spaces. In the proof, we omit the dependence of constants on $L(K)$.

Step 1: Construction of $T_{a}: \dot{W}^{-1, p} \rightarrow L^{p}(1<p<d)$. We compensate for the lack of smallness of $a$ by adding a weight $w=2^{-\phi}$ where $\phi$ is a smooth bounded increasing radial function. The goal is to insure that

$$
\|\operatorname{Tad}(a)\|_{L_{w}^{p} \rightarrow L_{w}^{p}} \ll 1
$$

We denote

$$
A_{k}=\left\{x \in \mathbb{R}^{d}: k \leq \phi(x) \leq k+1\right\} .
$$

Then for $j \geq k$, by Hölder's inequality, the embedding $L^{q} \hookrightarrow \dot{W}^{-1, p}$ (where $q^{-1}=p^{-1}+d^{-1}$ ) and Proposition 4.1 we have

$$
\left\|1_{A_{j}} \operatorname{Tad}(a) 1_{A_{k}}\right\|_{L_{w}^{p} \rightarrow L_{w}^{p}} \lesssim 2^{k-j}\|a\|_{L^{d}\left(A_{k}\right)} .
$$


On the other hand, the LHS vanishes when $j<k$ by the exterior support property. After summation, we obtain

$$
\|\operatorname{Tad}(a)\|_{L_{w}^{p} \rightarrow L_{w}^{p}} \lesssim \sup _{k}\|a\|_{L^{d}\left(A_{k}\right)} .
$$

Thus to insure the desired smallness, it suffices to choose $w$ so that the RHS is small, which is easily done.

Step 2: Boundedness into $\dot{H}^{\frac{d-4}{2}}$. Let $n$ be the least integer greater than or equal to $\frac{d-4}{2}$. The strategy is to commute $\partial$ for up to order $n$ (as in Step 2 in the proof of Proposition 4.2), and inductively prove boundedness of $T_{a}: \dot{W}^{m-1, \frac{d}{m+2}} \rightarrow \dot{W}^{m, \frac{d}{m+2}}$ for $m=1, \ldots, n$; this would directly imply (1.13) for even $d$, and after interpolation for odd $d$.

For simplicity, as in Step 2 of the proof of Proposition 4.2, we only consider the case $n=1$; the general case is dealt with by induction in a similar manner. Our starting point is (4.5):

$$
\partial e=T\left(\partial h-\left[a^{\ell}, \partial e_{\ell}\right]\right)-T\left(\left[\partial a^{\ell}, e_{\ell}\right]\right)+[\partial, T]\left(h-\left[a^{\ell}, e_{\ell}\right]\right) .
$$

The strategy is to use $\|e\|_{L^{d}}$, which is already under control, to estimate the last two terms, and use an iteration argument in $L_{w}^{p}$ as in Step 1 with $p=\frac{d}{3}$ to estimate $d e$. By Proposition 4.1. Sobolev and Hölder, we have

$$
\left\|T\left(\left[\partial a^{\ell}, e_{\ell}\right]\right)\right\|_{L^{\frac{d}{3}}} \lesssim\|\partial a\|_{L^{\frac{d}{2}}}\|e\|_{L^{\frac{d}{2}}} \lesssim\|a\|_{\dot{H}^{\frac{d-2}{2}}}\|e\|_{L^{\frac{d}{2}}} \cdot
$$

On the other hand, note that $[T, \partial]=\sum_{\omega} T_{\omega} \partial \chi_{\omega}$ (cf. proof of Proposition 4.1), where $\chi_{\omega}$ is 0-homogeneous. Thus by $T_{\omega}: \dot{W}^{-1, p} \rightarrow L^{p}$, Hardy, Sobolev and Hölder,

$$
\left\|[\partial, T]\left(h-\left[a^{\ell}, e_{\ell}\right]\right)\right\|_{L^{\frac{d}{3}}} \lesssim\left\|h-\left[a^{\ell}, e_{\ell}\right]\right\|_{L^{\frac{d}{3}}} \lesssim\|h\|_{L^{\frac{d}{3}}}+\|a\|_{\dot{H}^{\frac{d-2}{2}}}\|e\|_{L^{\frac{d}{2}}} \cdot
$$

By Step 1 with $p=\frac{d}{2}$, we have $\|e\|_{L^{\frac{d}{2}}} \lesssim\|h\|_{\dot{W}^{-1, \frac{d}{2}}} \lesssim\|h\|_{L^{\frac{d}{3}}}$. Then finding the fixed point $\partial e$ of (4.6) as in Step 1, the desired estimate $\|\partial e\|_{L^{\frac{d}{3}}} \lesssim\|a\|_{\dot{H} \frac{d-2}{2}}\|h\|_{L^{\frac{d}{3}}}$ follows.

Step 3: Higher regularity. This step is analogous to Step 2 of Proposition 4.2, where the iteration is done in $L_{w}^{p}$.

4.2. Initial data surgery. Now we explore consequences of the previous result in terms of excising and extending initial data sets. The aim of this subsection is to prove Theorems 1.16 and 1.17 .

Before we turn to the proofs, a few remarks about Sobolev extension are in order. For any domain $K$ with locally Lipschitz boundary, Stein's extension theorem [28, §VI.3] says that there exists a universal linear extension operator $\mathfrak{E}$ for all Sobolev spaces $W^{\sigma, p}(K) \rightarrow$ $W^{\sigma, p}\left(\mathbb{R}^{d}\right)$. When $K$ is convex with $R(K)=1$ (which we may insure by scaling), it can be checked that the constant depends only on $\sigma, p$ and the Lipschitz constant $L(K)$. In particular, we have

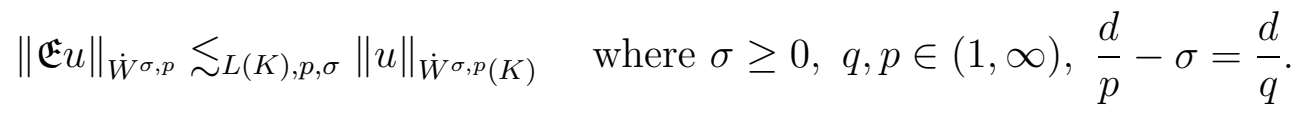

The same bound holds for general $R(K)$ by scaling-invariance of the both sides. Similarly, for an annular region $4 K \backslash \bar{K}$ (with general $R(K)$ ), there exists a universal linear extension

\footnotetext{
${ }^{8}$ As in Step 2 of Proposition 4.2, to be rigorous one should work with divided differences, but the argument is essentially the same.
} 
operator $\mathfrak{E}$ such that

$$
\|\mathfrak{E} u\|_{\dot{W}^{\sigma, p}} \lesssim_{L(K), p, \sigma}\|u\|_{\dot{W}^{\sigma, p}(4 K \backslash \bar{K})} \quad \text { where } \sigma \geq 0, q, p \in(1, \infty), \frac{d}{p}-\sigma=\frac{d}{q} .
$$

Now we prove Theorem 1.16, concerning truncation of Yang-Mills initial data sets.

Proof of Theorem 1.16. Let $(a, e)$ be the given $\mathcal{H}^{\frac{d-2}{2}}$ Yang-Mills initial data set on $2 K \backslash \bar{K}$. In this proof, we use the shorthands

$$
A=\|a\|_{\dot{H} \frac{d-2}{2}(2 K \backslash \bar{K})}, \quad E=\|e\|_{\dot{H}^{\frac{d-4}{2}}(2 K \backslash \bar{K})} .
$$

First, we use the universal extension $\mathfrak{E}$ to extend $a, e$ to $\bar{a}, \bar{e}^{\prime}$ on $\mathbb{R}^{d}$, respectively. Clearly, restriction of $\bar{a}$ satisfies (1.14). On the other hand, $\bar{e}^{\prime}$ obeys a favorable bound, but violates the Gauss equation outside $2 K \backslash \bar{K}$. More precisely,

$$
\left(\mathbf{D}^{(\bar{a})}\right)^{\ell} \bar{e}_{\ell}^{\prime}=h
$$

where $h=0$ in $2 K \backslash \bar{K}$ since $(\bar{a}, \bar{e})=(a, e)$ there. Let $\chi_{\text {out }}$ be a smooth cutoff which equals zero in $K$ and 1 outside $2 K$, then let $h_{\text {out }}=\chi_{\text {out }} h$. Note that

$$
\left\|h_{\text {out }}\right\|_{\dot{H}^{\frac{d-6}{2}}} \lesssim\|\partial \bar{e}\|_{\dot{H}^{\frac{d-6}{2}}}+\|\bar{a}\|_{\dot{H}^{\frac{d-2}{2}}}\|\bar{e}\|_{\dot{H}^{\frac{d-4}{2}}} \lesssim_{A} E .
$$

Hence, by Theorem 1.14, we find $d_{\ell}$ such that $\left(\mathbf{D}^{(\bar{a})}\right)^{\ell} d_{\ell}=-h_{\text {out }}, d=0$ in $2 K$ and $\|d\|_{\dot{H}^{\frac{d-4}{2}}} \lesssim_{A}$ $E$. The desired $\bar{e}$ is then given by the restriction of $\bar{e}^{\prime}+d$ to $\mathbb{R}^{d} \backslash \bar{K}$.

To conclude the proof, note that the higher regularity and local Lipschitz properties are obvious by construction. Finally, equivariance under constant gauge transformations can be insured by fixing a particular construction, conjugating by elements of $\mathbf{G}$, and then averaging over $\mathbf{G}$.

Combined with Uhlenbeck's lemma (Theorem 3.11), we may now prove the final excisionand-extension result (Theorem 1.17).

Proof of Theorem 1.17. We only treat the case when $d \geq 4$ is even and $X=B_{R}$. The other cases are simpler and thus are left to the reader (when $d$ is odd, Uhlenbeck's lemma is not needed, and when $X=\mathbb{R}^{d}$, the extension procedure is unnecessary).

Step 1: Application of Uhlenbeck's lemma. As in the proof of Proposition 3.19, we first set $a_{r}=0$ by Lemma 3.5, and extend $a$ outside $B_{R}$ by Lemma 3.18. Then the $L^{d_{-}}$ concentration radius of $a$ does not vary much, and Uhlenbeck's lemma (Theorem 3.11 ) is applicable on any ball $B_{2 r}(x)$ with $r<10 r_{c}$ and $x \in B_{R}$. We claim that

$$
\|\tilde{a}\|_{\dot{H}^{\frac{d-2}{2}}\left(B_{r}(x) \cap X\right)} \lesssim\left\|\mathbf{D}^{\left(\frac{d-4}{2}\right)} F[a]\right\|_{L^{2}\left(B_{r}(x) \cap X\right)}+\|F[a]\|_{L^{\frac{d}{2}}\left(B_{r}(x) \cap X\right)} .
$$

For interior balls (i.e., $B_{2 r}(x) \cap \partial B=\emptyset$ ), this bound follows directly from Lemma 3.13. For boundary balls (i.e., $B_{2 r}(x) \cap \partial B \neq \emptyset$ ), we obtain angular regularity (with respect to the center of $B_{R}$ ) by Lemma 3.14, then radial regularity by (3.11). We note that the implicit constant is controlled thanks to the smallness of $\epsilon_{*}$.

Next, by the formula $\left.O_{; x}=A d(O) a-\tilde{a}\right)$, we obtain (1.18). Then it also follows that

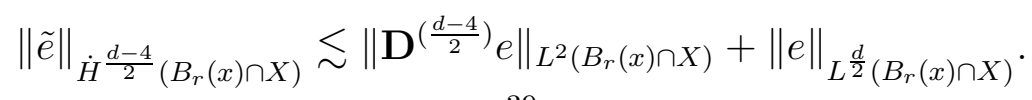


Step 2: Application of Theorem 1.16. We apply Theorem 1.16 to $(\tilde{a}, \tilde{e})$ and obtain an extended Yang-Mills initial data set outside the convex domain $K=B_{r}(x) \cap B_{R}$, which we still denote by $(\tilde{a}, \tilde{e})$. We note for domains of the form $K=B_{r}(x) \cap B_{R}$, we have the universal bound $R(K) \simeq r$ and $L(K) \simeq 1$. Therefore, by (1.14), (1.15), and the preceding bounds for $(\tilde{a}, \tilde{e})$ on $K$, we obtain (1.17).

Step 3: Completion of proof. It remains to prove Theorem 1.17,(2). We begin by clarifying the ambiguity of the construction so far. In Step 1, the triple $(\tilde{a}, \tilde{e}, O) \uparrow_{K}$ is determined up to a constant gauge transformation, as in Uhlenbeck's lemma (Theorem 3.11 ). Since Theorem 1.16 is equivariant under such operations, the corresponding extensions $(\tilde{a}, \tilde{e})$ are also constant gauge transformations of each other.

As a result, in order to prove (2), it suffices to show that we can enforce strong convergence of $\left(\tilde{a}^{n}, \tilde{e}^{n}, O^{n}\right)$ to $(\tilde{a}, \tilde{e}, O)$ in $H^{\frac{d-2}{2}} \times H^{\frac{d-4}{2}} \times H^{\frac{d}{2}}(K)$, after passing to a subsequence and conjugating the sequence with a constant gauge transformation. Proceeding as in Theorem 3.11. (2), we may first insure convergence of a suitable subsequence up to a constant gauge transformation in $W^{1, \frac{d}{2}} \times L^{\frac{d}{2}} \times W^{2, \frac{d}{2}}(K)$. Then by Remark 3.15, strong convergence in the desired topology (of the same sequence) may be proved. We omit the straightforward details.

\section{The LOCAL THEORY FOR The HyPERBoliC YANG-Mills EQUATiON}

In this section, we consider the local-in-time theory for the hyperbolic Yang-Mills equation for data in an arbitrary topological class.

5.1. Gauge equivalent classes of connections. We start by verifying that the gaugeequivalent class of $\mathcal{H}^{\frac{d-2}{2}}$ connections is closed, as asserted in Section 1.7.

Proposition 5.1. Let $A$ be an $\mathcal{H}_{\text {loc }}^{\frac{d-2}{2}}$ connection in $\mathcal{O} \subseteq \mathbb{R}^{1+d}$. Then $[A]$ is closed in the corresponding topology.

Proof. Suppose that $O^{(n)}$ is a sequence of admissible gauge transformations so that the gauge equivalent connections $A^{(n)}$ given by

$$
A^{(n)}=A d\left(O^{(n)}\right) A-O_{; t, x}^{(n)}
$$

converge to an $\mathcal{H}_{l o c}^{\frac{d-2}{2}}$ connection $B$. Then we need to show that $A$ and $B$ are gauge equivalent.

We first consider the corresponding gauge transformations $O^{(n)}$. By the relation (5.1), it follows that these are uniformly bounded on compact sets. Hence, by compact Sobolev embeddings we obtain a limiting gauge transformation $O$, so that on a subsequence we have

(i) $O$ satisfies the bounds

$$
\nabla O \in L^{\infty} H_{l o c}^{\frac{d-2}{2}}
$$

(ii) Convergence in weaker topologies:

$$
\nabla O^{(n)} \rightarrow \nabla O \quad \text { in } L^{p} W_{l o c}^{\frac{d-2}{2}, 2-}, p<\infty .
$$

(iii) Pointwise a.e convergence:

$$
O^{(n)}(t, x) \rightarrow O(t, x) \text { a.e., } \quad \nabla O^{(n)}(t, x) \rightarrow \nabla O(t, x) \text { a.e.. }
$$


These properties allow us to pass to the limit and obtain

$$
B=O A O^{-1}-O_{; t, x}
$$

as well as the similar relation for the curvatures.

It remains to improve the first property (i) above to continuity in time. This cannot come from weak convergence, instead it is a consequence of the corresponding continuity property for $A$ and $B$. We start from property (ii), which guarantees that $O(t, x)$ is continuous in $t$ for almost every $x$. Since $A, B \in C_{t} L_{l o c}^{d}$, so is $A d(O) A$ and thus $\nabla O$. We now differentiate and repeat the process for $\partial \nabla O$ in $L^{\frac{d}{2}}$, and so on.

5.2. Local theory at optimal regularity for dimensions $d \geq 4$. We begin by recalling the temporal gauge small data global well-posedness result proved 9 in [21].

Theorem 5.2 ([21, Theorem 1.17]). If the $\dot{H}^{\frac{d-2}{2}} \times \dot{H}^{\frac{d-4}{2}}$ norm of the initial data set $(a, e)$ is smaller than some universal constant $\epsilon_{*}$, then the corresponding solution $\left(A_{t, x}, \partial_{t} A_{t, x}\right)$ in the temporal gauge $A_{0}=0$ exists globally in $C_{t}\left(\mathbb{R} ; \dot{H}^{\frac{d-2}{2}} \times \dot{H}^{\frac{d-4}{2}}\right)$, and obeys the a-priori bound

$$
\left\|\nabla A_{x}\right\|_{L^{\infty} \dot{H}^{\frac{d-4}{2}}} \lesssim\|(a, e)\|_{\dot{H}^{\frac{d-2}{2}} \times \dot{H}^{\frac{d-4}{2}}} .
$$

The solution is unique among the local-in-time limits of smooth solutions, and it depends continuously on the data $(a, e) \in \dot{H}^{\frac{d-2}{2}} \times \dot{H}^{\frac{d-4}{2}}$.

We now derive Theorem 1.22 from Theorems 1.17 and 5.2 ,

Proof of Theorem 1.22. The idea is to construct the local-in-spacetime solutions using Theorems 1.17 and 5.2, and then patch up by finite speed of propagation (i.e., local-in-spacetime uniqueness) in the temporal gauge.

Step 1: Construction of local-in-spacetime solutions. Consider a ball $B_{r}(x)$ with $r<10 r_{c}$ and $x \in X$; we introduce the abbreviation $K=B_{r}(x) \cap X$. Let $(\tilde{a}, \tilde{e})$ and $O$ be the global Yang-Mills initial data and the gauge transformation associated with $(a, e)$ by Theorem 1.17. (1); recall that $(a, e)$ is gauge equivalent to $(\tilde{a}, \tilde{e})$ via $O$ on $K$. Choosing $\epsilon_{*}$ sufficiently small, Theorem 5.2 produces a unique $C_{t} \mathcal{H}^{\frac{d-2}{2}}$ temporal-gauge solution $\tilde{A}$ corresponding to $(\tilde{a}, \tilde{e})$. We define $A$ on $\mathcal{D}(K)$ by

$$
A_{\mu}(t, x)=A d\left(O^{-1}(x)\right) \tilde{A}_{\mu}(t, x)-O_{; \mu}^{-1}(x) .
$$

Note that $(\tilde{a}, \tilde{e}, O)$ in Theorem 1.17. (1) is determined up to a constant gauge transformation, but any choice leads to the same solution $A$. By (1.17), (1.18) and Theorem 5.2, it follows that

$$
\left\|\nabla A_{x}\right\|_{L^{\infty} \dot{H} \frac{d-4}{2}(\mathcal{D}(K))} \lesssim\|(a, e)\|_{\dot{H}^{\frac{d-2}{2}} \times \dot{H}^{\frac{d-4}{2}}(K)} .
$$

Step 2: Continuous dependence and uniqueness. We claim that the mapping

$$
\mathcal{H}^{\frac{d-2}{2}}(K) \ni(a, e) \mapsto\left(A, \partial_{t} A\right) \in C_{t}\left(H^{\frac{d-2}{2}} \times H^{\frac{d-4}{2}}\right)(\mathcal{D}(K))
$$

is continuous. Indeed, for the purpose of contradiction, suppose that there is a sequence of $\mathcal{H}^{\frac{d-2}{2}}$ Yang-Mills initial data sets on $K$ such that $\left(a^{n}, e^{n}\right) \rightarrow(a, e)$, while $\left(A^{n}, \partial_{t} A^{n}\right) \not \rightarrow$ $\left(A, \partial_{t} A\right)$ in $C_{t}\left(H^{\frac{d-2}{2}} \times H^{\frac{d-4}{2}}\right)(\mathcal{D}(K))$. By passing to a subsequence, we may assume that no

\footnotetext{
${ }^{9}$ In [21, this theorem is stated an proved in the most difficult case $d=4$. Nevertheless, its proof may be extended to $d>4$.
} 
further subsequence of $\left(A^{n}, \partial_{t} A^{n}\right)$ converges to $\left(A, \partial_{t} A\right)$ in the same topology. However, by Theorem 1.17. (2) and the continuity statement in Theorem 5.2, there exists a subsequence for which $\left(\tilde{A}^{n}, \partial_{t} \tilde{A}^{n}\right) \rightarrow\left(\tilde{A}, \partial_{t} \tilde{A}\right)$ in $C_{t}\left(\dot{H}^{\frac{d-2}{2}} \times \dot{H}^{\frac{d-4}{2}}\right)$. By the convergence $O^{n} \rightarrow O$ and $\left(O^{n}\right)^{-1} \rightarrow O^{-1}$ in $\mathcal{G}^{\frac{d}{2}, 2}(K)$, it follows that $\left(A^{n}, \partial_{t} A^{n}\right) \rightarrow\left(A, \partial_{t} A\right)$ in the above topology, which is a contradiction.

From continuous dependence and persistence of regularity in Theorems 1.17 and 5.2 it follows that $\left(A, \partial_{t} A\right)$ defined in Step 1 is approximated by smooth (temporal gauge) solutions, i.e., it is a solution to (1.9) in the sense of Definition [1.19. Therefore, uniqueness of the solution on $\mathcal{D}(K)$ in the sense of Definition 1.19 in the temporal gauge follows.

Step 3: Conclusion of the proof. Consider now a family of balls $\left\{B_{2 r_{c}}(x)\right\}_{x \in X}$, and the corresponding family of temporal gauge solutions in each $\mathcal{D}\left(B_{2 r_{c}}(x) \cap X\right)$. By the localin-spacetime uniqueness that we just proved, these solutions coincide on the intersections, and therefore define a unique temporal gauge solution (in the sense of Definition 1.19) in $\mathcal{D}_{\left[0, r_{c}\right)}(X) \subseteq \cup_{x \in X} \mathcal{D}\left(B_{2 r_{c}}(x) \cap X\right)$.

Properties (1) and (2) claimed in Theorem 1.22 follow from the construction. For the a-priori bound in (3), we repeat the above steps to the data restricted to uniformly spaced balls $B$ of radius $2 r_{c}$ that cover $B_{R^{\prime}}(x)$. By local-in-spacetime uniqueness, the result coincides with $A$ in $\mathcal{D}_{\left[0, r_{c}\right)}\left(B_{R^{\prime}}(x)\right)$. Moreover, (1.20) follows by summing up the a-priori bounds in Theorem 5.2 for the local-in-spacetime solutions.

Next, we also show that all $\mathcal{H}_{l o c}^{\frac{d-2}{2}}$ solutions (in the sense of Definition 1.19) are gauge equivalent to the corresponding temporal solutions.

Proof of Theorem 1.23. Let $A^{(n)}$ be a sequence of smooth solutions which converge to $A$ in the norm $C_{t}\left(H_{l o c}^{\frac{d-2}{2}} \times H_{l o c}^{\frac{d-4}{2}}\right)$. Let $\tilde{A}^{(n)}$, respectively $\tilde{A}$, be the corresponding temporal solutions. We know that $\tilde{A}^{(n)}$ and $A^{(n)}$ are gauge equivalent; denote by $O^{(n)}$ the corresponding gauge transformations.

We know that in the $H^{1}$ topology

$$
A d\left(O^{(n)}\right) \tilde{A}^{(n)}-O_{; t, x}^{(n)}=A^{(n)} \rightarrow A
$$

but also that

$$
\tilde{A}^{(n)} \rightarrow \tilde{A}
$$

Thus, the gauge transformations $O^{(n)}$ satisfy uniform bounds locally. Then it follows that (up to a subsequence)

$$
\operatorname{Ad}\left(O^{(n)}\right) \tilde{A}-O_{; t, x}^{(n)} \rightarrow A .
$$

But now we can use Proposition 5.1 to conclude that $\tilde{A}$ and $A$ are gauge equivalent.

Continuity of $A_{x}(t)$ in $\mathcal{H}_{l o c, r_{c}}^{\frac{d-2}{2}}$, as stated in Theorem 1.22, is in general insufficient to conclude invariance of the topological class. However, combined with finite speed of propagation and Proposition 1.9, we may nevertheless prove that the topological class of $A_{x}(t)$ is conserved under the hyperbolic Yang-Mills evolution.

Proof of Proposition 1.24. Thanks to Theorem 1.23, it suffices to consider a temporal gauge solution $A_{x}(t)$. By a usual continuous induction in $t$ (as well as time reversibility of (1.9)), it suffices to show that the $\left[A_{x}(t)\right]=\left[A_{x}(0)\right]$ for all $t>0$ sufficiently close to 0 . 
Since $\mathcal{E}_{\mathbb{R}^{d}}^{\frac{d-2}{2}}(a, e)<\infty$, there exists $R>0$ such that $\mathcal{E}_{\mathbb{R}^{d} \backslash \overline{B_{R}}}^{\frac{d-2}{2}}(a, e) \ll \epsilon_{*}$. By Uhlenbeck's lemma (when $d$ is even) and the local-in-spacetime a-priori estimate (1.20), it follows that

$$
\sup _{t \in\left[0, r_{c}\right)} \mathcal{E}_{\mathbb{R}^{d} \backslash \overline{B_{R+t}}}^{\frac{d-2}{2}}\left(A_{x}(t), \partial_{t} A_{x}(t)\right) \lesssim \mathcal{E}_{\mathbb{R}^{d} \backslash \overline{B_{R}}}^{\frac{d-2}{2}}(a, e) \ll \epsilon_{*}
$$

In particular, choosing $R$ large enough, we may insure that

$$
\left\|F\left[A_{x}(t)\right]\right\|_{L^{\frac{d}{2}}\left(\mathbb{R}^{d} \backslash \overline{B_{2 R}}\right)}<\epsilon_{*}
$$

where $\epsilon_{*}$ is as in Proposition 1.9. For $t>0$ sufficiently close to 0 , by the continuity property (1.19), we may also insure that

$$
\left\|A_{x}(t)-A_{x}(0)\right\|_{L^{d}\left(B_{2 R}\right)}<\epsilon_{*}
$$

By Proposition [1.9, it follows that $\left[A_{x}(t)\right]=\left[A_{x}(0)\right]$.

Finally, we turn to the proof of Theorem 1.25. The main ingredient is the caloric gauge small data well-posedness theorem from [21]:

Theorem 5.3 ([21, Corollary 1.13]). Let $(a, e)$ be an Yang-Mills initial data set with the property that its $\dot{H}^{\frac{d-2}{2}} \times \dot{H}^{\frac{d-4}{2}}$ norm is smaller than some universal constant $\epsilon_{*}^{2}$. Then there exists a gauge transformation $O \in \dot{H}^{\frac{d}{2}}\left(\mathbb{R}^{d} ; \mathbf{G}\right)$ of $(a, e)$ to a caloric gauge data $(\tilde{a}, \tilde{e})$, which is unique up to a constant gauge transformation. Moreover, the corresponding caloric gauge solution $\left(\tilde{A}_{t, x}, \partial_{t} \tilde{A}_{t, x}\right)$ exists globally in time, and obeys the a-priori bound

$$
\left\|\tilde{A}_{x}\right\|_{S^{\frac{d-2}{2}}} \lesssim\|(a, e)\|_{\dot{H}^{\frac{d-2}{2}} \times \dot{H}^{\frac{d-4}{2}}} .
$$

We refer the reader to [20, 21] for the precise definition of the caloric gauge and the $S^{\frac{d-2}{2}}$ norm. For our purposes, all we need to know is that

$$
\left\|\nabla \tilde{A}_{x}\right\|_{L^{\infty} \dot{H} \frac{d-4}{2}}+\left\|\tilde{A}_{x}\right\|_{L^{2} L^{2 d}} \lesssim\left\|\tilde{A}_{x}\right\|_{S^{\frac{d-2}{2}}}
$$

and that the a-priori bound of the $S^{\frac{d-2}{2}}$ norm implies the following additional control of the solution $\tilde{A}_{t, x}$ [21, Theorem 5.1]:

$$
\left\|\square \tilde{A}_{x}\right\|_{\ell^{1} L^{2} \dot{H} \frac{d-5}{2}}+\left\|\partial^{\ell} \tilde{A}_{\ell}\right\|_{\ell^{1} L^{2} \dot{H} \frac{d-3}{2}^{2}}+\left\|\nabla \tilde{A}_{0}\right\|_{\ell^{1} L^{2} \dot{H}^{\frac{d-3}{2}}} \lesssim\left\|\tilde{A}_{x}\right\|_{S^{\frac{d-2}{2}}}\left\|\tilde{A}_{x}\right\|_{S^{\frac{d-2}{2}}}^{2} .
$$

Combined with the initial data surgery technique (Theorem 1.17) and the patching procedure in Section 3.2, we may now prove Theorem 1.25,

Proof of Theorem 1.25. On the one hand, we have a global $\mathcal{H}_{l o c}^{\frac{d-2}{2}}$ solution $A$ in $\mathcal{D}_{\left[0, r_{c}\right)}\left(B_{R}\right)$ by Theorem 1.22, On the other hand, we can cover $\left[0, r_{c}\right) \times B_{R-4 r_{c}}$ with cylinders $\left[0, r_{c}\right) \times B_{r_{c}}\left(x_{\alpha}\right)$, each of which is contained in a truncated cone $\mathcal{D}_{\left[0, r_{c}\right)}\left(B_{4 r_{c}}\left(x_{\alpha}\right)\right)$ whose base is contained in $B_{R}$, i.e., $B_{4 r_{c}}\left(x_{\alpha}\right) \subseteq B_{R}$. In each $\mathcal{D}_{\left[0, r_{c}\right)}\left(B_{4 r_{c}}\left(x_{\alpha}\right)\right)$, by Theorem 5.3, we have a gauge-equivalent caloric solution $\tilde{A}_{(\alpha)}$ satisfying

$$
\left\|\nabla \tilde{A}_{(\alpha) x}\right\|_{L^{\infty} \dot{H} \frac{d-4}{2}}+\left\|\square \tilde{A}_{(\alpha) x}\right\|_{\ell^{1} L^{2} \dot{H} \frac{d-5}{2}}+\left\|\partial^{\ell} \tilde{A}_{(\alpha) \ell}\right\|_{\ell^{1} L^{2} \dot{H} \dot{H}^{\frac{d-3}{2}}}+\left\|\nabla \tilde{A}_{(\alpha) 0}\right\|_{\ell^{1} L^{2} \dot{H} \frac{d-3}{2}} \lesssim \epsilon_{*} .
$$

In the remainder of the proof, we restrict each solution $\tilde{A}_{(\alpha)}$ to the cylinder $\left[0, r_{c}\right) \times B_{r_{c}}\left(x_{\alpha}\right)$. 
We need to compute the regularity of the gauge transformation $O_{(\alpha \beta)}$ between two such solutions $\tilde{A}_{(\alpha)}$ and $\tilde{A}_{(\beta)}$. We build up the regularity of $O_{(\alpha \beta)}$ in several stages, depending on the formula

$$
\tilde{A}_{(\alpha)}=A d\left(O_{(\alpha \beta)}\right) \tilde{A}_{(\beta)}-O_{(\alpha \beta) ; t, x} \quad \text { in }\left[0, r_{c}\right) \times\left(B_{r_{c}}\left(x_{\alpha}\right) \cap B_{r_{c}}\left(x_{\beta}\right)\right)
$$

In what follows, all norms are over $\left[0, r_{c}\right) \times\left(B_{r_{c}}\left(x_{\alpha}\right) \cap B_{r_{c}}\left(x_{\beta}\right)\right)$, and we omit the subscripts $(\alpha),(\beta)$ and $(\alpha \beta)$.

(i) $L^{p}$ regularity. It immediately follows that

$$
O_{; x}, O_{; t} \in L^{\infty} L^{d} \cap L^{2} L^{2 d} .
$$

Reiterating this, we also obtain

$$
O_{; x}, O_{; t} \in L^{\infty} \dot{H}^{\frac{d-2}{2}} .
$$

(ii) $\ell^{1}$ Besov structure for $O_{; x}$. Here we obtain

$$
O_{; x} \in \ell^{1}\left(L^{\infty} \dot{H}^{\frac{d-2}{2}} \cap L^{2} \dot{H}^{\frac{d-1}{2}}\right) .
$$

which follows from the div-curl system 10 for $O_{; x}$ (cf. Lemma 3.16).

(iii) $\ell^{1}$ Besov structure for $O_{; t}$. Next, we obtain

$$
O_{; t} \in \ell^{1}\left(L^{\infty} \dot{H}^{\frac{d-2}{2}} \cap L^{2} \dot{H}^{\frac{d-1}{2}}\right) .
$$

which is obtained by differentiating in $x$ in the $O_{; t}$ relation. Differentiating instead in $t$, we also obtain

$$
\partial_{t} O_{; t} \in \ell^{1}\left(L^{\infty} \dot{H}^{\frac{d-4}{2}} \cap L^{2} \dot{H}^{\frac{d-3}{2}}\right) .
$$

(iv) $\square O_{; x} \in \ell^{1} L^{2} \dot{H}^{\frac{d-5}{2}}$. This requires a similar bound for $\left[O_{; \alpha}, \partial^{\alpha} \tilde{A}\right]$ and for $\left[\partial^{\alpha} O_{; \alpha}, \tilde{A}\right]$. Both of them follow from the previous bounds.

To summarize, we have the regularity properties:

$$
O_{; x} \in \ell^{1} L^{2} \dot{H}^{\frac{d-1}{2}}, \quad \partial_{t}^{2} O_{; x} \in \ell^{1} L^{2} \dot{H}^{\frac{d-5}{2}}, \quad \nabla O_{; t} \in \ell^{1} L^{2} \dot{H}^{\frac{d-3}{2}},
$$

where $\partial_{t}^{2} O_{; x} \in L^{2} \dot{H}^{\frac{d-5}{2}}$ follows by combining (ii) and (iii). These in particular imply that each $O$ is continuous, and is close to a constant in $L^{\infty}$. Hence, the operations of pointwise multiplication, inversion, adjoint action on $\mathfrak{g}$ etc. are all well-behaved for $O$ (in contrast to the general situation in Section 3.1).

Next step is to patch up the local gauges. Taking only the balls $B_{r_{c}}\left(x_{\alpha}\right)$ which cover $B_{R-4 r_{c}}$ and which are uniformly separated, Scenario (2) in Section 3.2 is applicable to each fixed time $\{t\} \times B_{R-4 r_{c}}$. Note that the diffeomorphisms and the smooth cutoffs involved in the patching procedure in Scenario (1) in Section 3.2 all depend trivially on $t$. It follows that on each $\left[0, r_{c}\right) \times B_{\alpha}^{\prime}$, the gauge transformations $P_{(\alpha)}$ obey

$$
P_{; x} \in \ell^{1} L^{2} \dot{H}^{\frac{d-1}{2}}, \quad \partial_{t}^{2} P_{; x} \in \ell^{1} L^{2} \dot{H}^{\frac{d-5}{2}}, \quad \nabla P_{; t} \in \ell^{1} L^{2} \dot{H}^{\frac{d-3}{2}},
$$

where the bound depends only on $R / r_{c}$ and $\epsilon_{*}$.

It remains to verify the bound (1.22) for the global gauge potential $A$, which is a consequence of (5.6), (5.7) and the formula (3.6) (it is easily extended to the 0-th component).

\footnotetext{
${ }^{10}$ In order to appeal to interior regularity, we may in fact start with local data on slightly larger balls $B_{2 r_{c}}\left(x_{\alpha}\right)$, then shrink their radii to $r_{c}$ at this stage. We omit this minor technical detail.
} 
Here, we only sketch the proof of $\square A_{x} \in \ell^{1} L^{2} \dot{H}^{\frac{d-5}{2}}$, which is the trickiest, and leave the remaining cases to the reader.

Recalling the formula (3.6), we have

$$
\square A_{x}=\sum \chi_{\alpha}\left(A d\left(P_{(\alpha)}\right) \square \tilde{A}_{(\alpha) x}-\square P_{; x}+\text { h.o.t. }\right) .
$$

The higher order terms, whose precise expression is omitted, are estimated by (5.8) and (5.6). . Moreover, $\square P_{; x}=-\partial_{t}^{2} P_{; x}+\Delta P_{; x} \in \ell^{1} L^{2} \dot{H}^{\frac{d-5}{2}}\left(\left[0, r_{c}\right) \times B_{\alpha}^{\prime}\right)$ by (15.8). Thanks to (5.8), $A d\left(P_{(\alpha)}\right)$ may be easily removed in $\ell^{1} L^{2} \dot{H}^{\frac{d-5}{2}}\left(\left[0, r_{c}\right) \times B_{\alpha}^{\prime}\right)$. Then finally, $\square \tilde{A}_{(\alpha) x} \in$ $\ell^{1} L^{2} \dot{H}^{\frac{d-5}{2}}\left(\left[0, r_{c}\right) \times B_{\alpha}^{\prime}\right)$ by $(\underline{5.6})$.

5.3. Local theory in dimension $d=3$. Here we sketch the proofs of Theorems 1.27 and 1.28. The key result is the following subcritical initial data surgery result (cf. Theorems 1.16 and 1.17):

Theorem 5.4. Let $\frac{1}{2}<\sigma<\frac{5}{2}$, and let $(a, e)$ be an $\mathcal{H}^{\sigma}$ Yang-Mills initial data set on a convex domain $K$ in $\mathbb{R}^{3}$ satisfying

$$
\|a\|_{\dot{H}^{\frac{1}{2}(K)}} \leq \epsilon
$$

If $\epsilon>0$ is sufficiently small depending on $L(K)$, then there exists an $\mathcal{H}^{\sigma}$ Yang-Mills initial data set $(\bar{a}, \bar{e})$ in $\mathbb{R}^{3}$ that coincides with $(a, e)$ on $K$ and obeys

$$
\|\bar{a}\|_{\dot{H}^{\sigma} \cap R(K)^{-\sigma} L^{2}}+\|\bar{e}\|_{\dot{H}^{\sigma-1}+R(K)^{\sigma-1} L^{2}} \lesssim L(K)\|a\|_{\dot{H}^{\sigma} \cap R(K)^{-\sigma} L^{2}(K)}+\|e\|_{\dot{H}^{\sigma-1}+R(K)^{\sigma-1} L^{2}(K)} .
$$

It can be arranged so that the association $(a, e) \mapsto(\bar{a}, \bar{e})$ is equivariant under constant gauge transformations, and so that $(a, e) \mapsto(\bar{a}, \bar{e})$ is locally Lipschitz continuous. Moreover, if $(a, e)$ is smooth, then so is $(\bar{a}, \bar{e})$.

Proof. By rescaling, we set $R(K)=1$ so that $\dot{H}^{\sigma} \cap R(K)^{-\sigma} L^{2} \simeq H^{\sigma}$ and $\dot{H}^{\sigma-1}+R(K)^{\sigma-1} L^{2} \simeq$ $H^{\sigma-1}$. As in the proof of Theorem 1.16, we apply the universal extension operator $\mathfrak{E}$ to $(a, e)$ to first obtain $\left(\bar{a}, \bar{e}^{\prime}\right) \in H^{\sigma} \times H^{\sigma-1}\left(\mathbb{R}^{3}\right)$. Then the error for the Gauss equation $h=\left(\mathbf{D}^{(\bar{a})}\right)^{\ell} \bar{e}^{\prime}$ is supported outside $K$ and obeys $\|h\|_{H^{\sigma-2}} \lesssim_{\|\bar{a}\|_{H^{\frac{1}{2}}}}\|e\|_{H^{\sigma-1}(K)}$. Since

$$
\|\bar{a}\|_{\dot{H}^{\frac{1}{2}}} \lesssim L(K)\|a\|_{\dot{H}^{\frac{1}{2}(K)}} \leq \epsilon,
$$

Proposition 4.2 is applicable if $\epsilon>0$ is chosen sufficiently small. Thus $d=-T_{\bar{a}} h$ satisfies

$$
\left(\mathbf{D}^{(\bar{a})}\right)^{\ell} d_{\ell}=-h, \quad\|d\|_{H^{\sigma-1}} \lesssim\|h\|_{H^{\sigma-2}} \lesssim\left\|\bar{e}^{\prime}\right\|_{H^{\sigma-1}},
$$

and vanishes in $K$. It follows that $\left(\bar{a}, \bar{e}=\bar{e}^{\prime}+d\right)$ is a Yang-Mills initial data set obeying the desired bound (5.10). The higher regularity and local Lipschitz properties are obvious by construction. Finally, equivariance under constant gauge transformations can be insured by fixing a particular construction, conjugating by elements of $\mathbf{G}$, and then averaging.

Next, we recall the temporal gauge small data local well-posedness of Tao.

Theorem $5.5([29])$. Let $\sigma>\frac{3}{4}$. If the $\mathcal{H}^{\sigma}$ norm of the initial data set $(a, e)$ is sufficiently small, then the corresponding solution $\left(A_{t, x}, \partial_{t} A_{t, x}\right)$ in the temporal gauge $A_{0}=0$ exists in $C_{t}\left((-1,1) ; H^{\sigma} \times H^{\sigma-1}\right)$, and obeys the a-priori bound

$$
\left\|\left(A_{x}, \partial_{t} A_{x}\right)\right\|_{L^{\infty}\left(H^{\sigma} \times H^{\sigma-1}\right)} \lesssim\|(a, e)\|_{H^{\sigma} \times H^{\sigma-1}} .
$$


The solution is unique among the local-in-time limits of smooth solutions, and it depends in a locally Lipschitz manner on the data $(a, e) \in H^{\sigma} \times H^{\sigma-1}$.

Now we are ready to prove Theorem 1.27 .

Sketch of Proof of Theorem 1.27. As in the proof of Theorem 1.22, the idea is to patch together the small local-in-spacetime solutions constructed using Theorems 5.4 and 5.5 in the temporal gauge.

It suffices to consider $\frac{3}{4}<\sigma<\frac{5}{2}$. Observe that, by subcriticality, the $\mathcal{H}_{\text {loc }}^{\sigma}$ norm obeys the following one-sided scaling property:

$$
\left\|\left(a^{(\lambda)}, e^{(\lambda)}\right)\right\|_{\mathcal{H}_{\text {loc }}^{\sigma}} \lesssim \lambda^{\sigma-\frac{1}{2}}\|(a, e)\|_{\mathcal{H}_{\text {loc }}^{\sigma}} \quad \text { for } \lambda \leq 1 .
$$

Here $\left(a^{(\lambda)}, e^{(\lambda)}\right)(x)=\left(\lambda a, \lambda^{2} e\right)(\lambda x)$ is the invariant scaling. Choosing

$$
\lambda \simeq\left(\epsilon_{*}\|(a, e)\|_{\mathcal{H}_{\text {loc }}^{\sigma}}^{-1}\right)^{\frac{2}{\sigma-1}}
$$

we may insure that $\left\|\left(a^{(\lambda)}, e^{(\lambda)}\right)\right\|_{\mathcal{H}_{\text {loc }}^{\sigma}} \ll \epsilon_{*}$. Choosing $\epsilon_{*}>0$ sufficiently small, we may apply Theorem 5.4 to each $\left(a^{(\lambda)}, e^{(\lambda)}\right) \uparrow_{B_{2}(x)}$ to find an extension $\left(\bar{a}^{(\lambda)}, \bar{e}^{(\lambda)}\right)$, and then Theorem5.5 to this global-in-space small data to obtain a temporal gauge solution $A^{(\lambda)}$ on the time interval $(-1,1)$. Proceeding as in the proof of Theorem 1.22, we obtain a well-posed temporal gauge solution for $\left(a^{(\lambda)}, e^{(\lambda)}\right)$ on $(-1,1)$. By rescaling back, the theorem follows with an explicit lower bound $T \gtrsim\|(a, e)\|_{\mathcal{H}_{l o c}^{\sigma-1}}^{-\frac{2}{\sigma}}$.

Finally, Theorem 1.28 is an easy corollary of Uhlenbeck's lemma (at subcritical regularity) and Theorem 1.27.

Sketch of Proof of Theorem 1.28. By conservation of energy, it suffices to prove that the temporal gauge solution given by Theorem 1.27 exists on a interval of length $T\left(\|(F[a], e)\|_{L_{l o c}^{2}}\right)$, where $\|\cdot\|_{L_{l o c}^{2}}=\sup _{x \in \mathbb{R}^{3}}\|\cdot\|_{L^{2}\left(B_{1}(x)\right)}$. As before, we have the one-sided scaling property

$$
\left\|\left(F\left[a^{(\lambda)}\right], e^{(\lambda)}\right)\right\|_{L_{l o c}^{2}} \lesssim \lambda^{\frac{1}{2}} \sup _{x \in \mathbb{R}^{3}}\|(F[a], e)\|_{L_{l o c}^{2}} \quad \text { for } \lambda \leq 1 .
$$

Choosing $\lambda \simeq \epsilon_{*}\|(F[a], e)\|_{L_{\text {loc }}^{2}}^{-2}$, we may insure that the LHS is $\lesssim \epsilon_{*}$. In what follows, we work with the rescaled data $\left(a^{(\lambda)}, e^{(\lambda)}\right)$; we omit the superscript $(\lambda)$ for simplicity. For the rescaled data, we wish to show that the corresponding temporal gauge solution given by Theorem 1.27 exists on the unit time interval $[0,1)$.

Fix a unit ball $B=B_{1}\left(x_{0}\right)$. Applying Uhlenbeck's lemma [30, Theorem 1.3] (which is possible if we take $\epsilon_{*}$ sufficiently small), we find $O \in \mathcal{G}^{2,2}(2 B)$ such that

$$
\|O\|_{H^{2}(B)} \lesssim\|a\|_{H^{1}(2 B)},
$$

and $(\tilde{a}, \tilde{e})=(A d(O) a-O ; x, A d(O) e)$ obeys

$$
\|(\tilde{a}, \tilde{e})\|_{H^{1} \times L^{2}(2 B)} \lesssim\|(F[a], e)\|_{L^{2}(2 B)} \lesssim \epsilon_{*} .
$$

By Theorem 5.5 (taking $\epsilon_{*}$ even smaller if necessary), we find a temporal gauge solution $\tilde{A}$ with data $(\tilde{a}, \tilde{e})$ on $(-1,1)$. Applying the $H^{2}(2 B)$ gauge transformation $O^{-1}$, we obtain a temporal gauge solution $A=A d\left(O^{-1}\right) \tilde{A}+O^{-1} O_{; t, x}$ in $\mathcal{D}_{[0,1)}(2 B)$. It can be easily verified that this solution is the limit of smooth temporal gauge solutions; hence it coincides with the solution given by Theorem 1.27 in $\mathcal{D}_{[0,1)}(2 B)$. Since this procedure can be applied to 
any unit ball $B \subseteq \mathbb{R}^{3}$, it follows that the temporal gauge solution exists on the time interval $[0,1)$, as desired.

\section{Harmonic YAng-Mills CONnECtions With COMPACT StRUCTURE Group}

The goal of this section is to prove Theorem 1.32. We proceed in two steps, in increasing generality.

Step 1: G is simple, compact and simply connected. Assume that $\mathbf{G}$ is compact and simply connected, and also that $\mathfrak{g}$ is simple, i.e., it is nonabelian $([\mathfrak{g}, \mathfrak{g}] \neq 0)$ and there is no nonzero proper ideal. As we will see, this case turns out to be completely analogous to the model case $\mathbf{G}=S U(2)$.

We need some algebraic preliminaries on compact simple Lie algebras over $\mathbb{R}$. We only sketch the part of the theory that is needed for us; for a more comprehensive treatment, see [12, Chapters II and IV].

A maximal abelian subalgebra $\mathfrak{h}$ of $\mathfrak{g}$ is called a Cartan subalgebra. Given such a $\mathfrak{h}$, consider $\{\operatorname{ad}(H): \mathfrak{g} \rightarrow \mathfrak{g}\}_{H \in \mathfrak{h}}$, which is a family of commuting anti-self-adjoint operators. Thus, viewed as linear operators on the complexification $\mathfrak{g}_{\mathbb{C}}=\mathfrak{g} \otimes_{\mathbb{R}} \mathbb{C}$, they are simultaneously diagonalizable with purely imaginary (or zero) eigenvalues. A nonzero linear functional $\alpha \in \mathfrak{h}^{*}$ is called a root 11 if the simultaneous eigenspace (called the root space)

$$
\mathfrak{g}_{\mathbb{C}, \alpha}=\left\{A \in \mathfrak{g}_{\mathbb{C}}: \operatorname{ad}(H) A=i \alpha(H) A, \forall H \in \mathfrak{h}\right\}
$$

is nonzero. We write $\Delta$ for the space of all roots. By the preceding discussion, we see that

$$
\mathfrak{g}_{\mathbb{C}}=\mathfrak{h}_{\mathbb{C}} \oplus \bigoplus_{\alpha \in \Delta} \mathfrak{g}_{\mathbb{C}, \alpha}
$$

as vector spaces. In particular, $\Delta \neq\{0\}$; in fact, it spans $\mathfrak{h}^{*}$. It is a fundamental result of Cartan that all Cartan subalgebras are related to each other by an $A d(O)$-action; thus $\Delta$ is independent of the choice of $\mathfrak{h}$.

To each $\alpha \in \Delta$, we use the inner product $\langle\cdot, \cdot\rangle$ to associate $H_{\alpha} \in \mathfrak{h}$ such that

$$
\alpha(H)=\left\langle H_{\alpha}, H\right\rangle, \quad H \in \mathfrak{h},
$$

and define the induced inner product on $\Delta$ by $\langle\alpha, \beta\rangle=\left\langle H_{\alpha}, H_{\beta}\right\rangle$. The roots with the largest norm are called the highest roots.

Clearly, if $\alpha \in \Delta$, then $-\alpha \in \Delta$ with $\mathfrak{g}_{\mathbb{C},-\alpha}=\overline{\mathfrak{g}_{\mathbb{C}, \alpha}}$. For any $E_{\alpha} \in \mathfrak{g}_{\mathbb{C}, \alpha}$, by definition,

$$
\left[H_{\alpha}, E_{\alpha}\right]=i \alpha\left(H_{\alpha}\right) E_{\alpha}=i\langle\alpha, \alpha\rangle E_{\alpha}, \quad\left[H_{\alpha}, \overline{E_{\alpha}}\right]=-i \alpha\left(H_{\alpha}\right) \overline{E_{\alpha}}=-i\langle\alpha, \alpha\rangle \overline{E_{\alpha}} .
$$

Moreover, $\operatorname{dim}_{\mathbb{C}} \mathfrak{g}_{\mathbb{C}, \alpha}=1$ and for any $E_{\alpha} \in \mathfrak{g}_{\mathbb{C}, \alpha}$, we have

$$
\left\langle E_{\alpha}, E_{\alpha}\right\rangle=0, \quad\left[E_{\alpha}, \overline{E_{\alpha}}\right]=i\left\langle E_{\alpha}, \overline{E_{\alpha}}\right\rangle H_{\alpha}
$$

where $\langle\cdot, \cdot\rangle$ is extended to $\mathfrak{g}_{\mathbb{C}}$ in a $\mathbb{C}$-bilinear fashion. For the proofs of the last properties, see [12, Section II.4].

Every root generates an embedding of $s u(2)$ into $\mathfrak{g}$. More precisely, given a root $\alpha \in \Delta$, normalize $E_{\alpha}$ so that

$$
\left\langle E_{\alpha}, \overline{E_{\alpha}}\right\rangle=\frac{2}{\langle\alpha, \alpha\rangle},
$$

\footnotetext{
${ }^{11} \mathrm{~A}$ more standard definition (used in [12]) is to define roots as $\alpha \in \mathfrak{h}_{\mathbb{C}}^{*}$ such that $\cap_{H \in \mathfrak{h}_{\mathbb{C}}} \operatorname{ker}(\operatorname{ad}(H)-$ $\alpha(H)) \neq\{0\}$. This differs from our definition by a factor of $i$.
} 
and consider $\mathbf{i}_{\alpha}, \mathbf{j}_{\alpha}, \mathbf{k}_{\alpha} \in \mathfrak{g}$ defined by

$$
\mathbf{i}_{\alpha}=\left(E_{\alpha}+\overline{E_{\alpha}}\right), \quad \mathbf{j}_{\alpha}=i\left(E_{\alpha}-\overline{E_{\alpha}}\right), \quad \mathbf{k}_{\alpha}=\frac{2}{\langle\alpha, \alpha\rangle} H_{\alpha} .
$$

Then it is straightforward to verify that $\left\{\mathbf{i}_{\alpha}, \mathbf{j}_{\alpha}, \mathbf{k}_{\alpha}\right\}$ generate an $s u(2)$-subalgebra, i.e.,

$$
\left[\mathbf{i}_{\alpha}, \mathbf{j}_{\alpha}\right]=2 \mathbf{k}_{\alpha}, \quad\left[\mathbf{j}_{\alpha}, \mathbf{k}_{\alpha}\right]=2 \mathbf{i}_{\alpha}, \quad\left[\mathbf{k}_{\alpha}, \mathbf{i}_{\alpha}\right]=2 \mathbf{j}_{\alpha}
$$

Indeed, (6.1) are precisely the Lie bracket relations satisfied the following standard basis of $s u(2)$ :

$$
\mathbf{i}=\left(\begin{array}{cc}
0 & 1 \\
-1 & 0
\end{array}\right), \quad \mathbf{j}=\left(\begin{array}{cc}
0 & i \\
i & 0
\end{array}\right), \quad \mathbf{k}=\left(\begin{array}{cc}
i & 0 \\
0 & -i
\end{array}\right)
$$

Note also that $\mathbf{i}_{\alpha}, \mathbf{j}_{\alpha}, \mathbf{k}_{\alpha}$ obeys

$$
\left|\mathbf{i}_{\alpha}\right|^{2}=\left|\mathbf{j}_{\alpha}\right|^{2}=\left|\mathbf{k}_{\alpha}\right|^{2}=\frac{4}{\langle\alpha, \alpha\rangle}
$$

By simplicity, all symmetric $A d$-invariant bilinear functions on $\mathfrak{g}$ (of which $\langle\cdot, \cdot\rangle$ is an example) are constant multiples of each other [12, Corollary 4.9]. Multiplying $\langle\cdot, \cdot\rangle$ by a suitable constant, which does not change the conclusion of Theorem 1.32, we may assume that:

$$
\text { The highest roots in } \mathfrak{g} \text { have }\langle\alpha, \alpha\rangle=2 \text {. }
$$

When $\mathbf{G}=S U(n)$, this amounts to taking $\langle A, B\rangle=-\operatorname{tr}(A B)$. We now recall the following well-known result of Bott [4] concerning the third homotopy group $\pi_{3}(\mathbf{G})$ of $\mathbf{G}$ :

Theorem 6.1. Let $\mathbf{G}$ be a simple, compact, simply connected Lie group. Then $\pi_{3}(\mathbf{G}) \simeq \mathbb{Z}$. Any Lie group homomorphism $\varphi: S U(2) \rightarrow \mathbf{G}$, induced by the Lie algebra homomorphism

$$
\mathrm{d} \varphi: s u(2) \rightarrow \mathfrak{g}, \quad(\mathbf{i}, \mathbf{j}, \mathbf{k}) \mapsto\left(\mathbf{i}_{\alpha}, \mathbf{j}_{\alpha}, \mathbf{k}_{\alpha}\right)
$$

for a highest root $\alpha$ in $\mathfrak{g}$, induces an isomorphism $\pi_{3}(S U(2)) \rightarrow \pi_{3}(\mathbf{G})$.

The identification $\pi_{3}(\mathbf{G}) \simeq \mathbb{Z}$ is due to Bott [4]. For the proof that such a $\varphi$ induces an isomorphism, see Atiyah-Hitchin-Singer [2, Section 8]. By our normalization (6.3), $\mathrm{d} \varphi$ is isometric.

Our goal now is to prove an analogue of Theorem 1.29 concerning topological classes, characteristic numbers and instantons. Let $a$ be a $\mathcal{A}_{\text {loc }}^{1,2}$ connection on $\mathbb{R}^{4}$ with finite energy, and let $O_{(\infty)}$ be a gauge at infinity for $a$ (which exists thanks to Theorem 1.5). By Theorem 6.1, $\left[O_{(\infty)}\right]=-\kappa[\varphi]$ for some $\kappa \in \mathbb{Z}$. We claim that:

Claim 6.2. We have $\chi=-8 \pi^{2} \kappa$. Moreover, there exists an instanton for each $\kappa$ with energy $8 \pi^{2}|\kappa|$.

To prove the claim, note that each self-dual (resp. anti-self-dual) $S U(2)$-connection $\tilde{a}_{\kappa}$ with second Chern number $c_{2}=-\kappa$ where $\kappa>0$ (resp. $\kappa<0$ ) induces a self-dual (resp. anti-self-dual) G-connection $a_{\kappa}=\mathrm{d} \varphi\left(\tilde{a}_{\kappa}\right)$ by the Lie algebra homomorphism $\mathrm{d} \varphi: s u(2) \rightarrow \mathfrak{g}$. Since $\mathrm{d} \varphi$ preserves the normalized $A d$-invariant inner product, which equals $-\operatorname{tr}(A B)$ on 
$s u(2)$, we have

$$
\begin{gathered}
\chi=\int_{\mathbb{R}^{4}}-\left\langle\mathrm{d} \varphi\left(F\left[\tilde{a}_{\kappa}\right]\right), \mathrm{d} \varphi\left(F\left[\tilde{a}_{\kappa}\right]\right)\right\rangle=\int_{\mathbb{R}^{4}} \operatorname{tr}\left(F\left[\tilde{a}_{\kappa}\right] \wedge F\left[\tilde{a}_{\kappa}\right]\right)=8 \pi^{2} c_{2} \\
\mathcal{E}_{e}\left(a_{\kappa}\right)=\frac{1}{2} \int_{\mathbb{R}^{4}}\left\langle\mathrm{~d} \varphi\left(F_{j k}\left[\tilde{a}_{\kappa}\right]\right), \mathrm{d} \varphi\left(F^{j k}\left[\tilde{a}_{\kappa}\right]\right)\right\rangle=\frac{1}{2} \int_{\mathbb{R}^{4}}-\operatorname{tr}\left(F_{j k}\left[\tilde{a}_{\kappa}\right] F^{j k}\left[\tilde{a}_{\kappa}\right]\right)=8 \pi^{2}\left|c_{2}\right| .
\end{gathered}
$$

Moreover, by a standard computation, the degree of a gauge at infinity $\tilde{O}_{\kappa(\infty)}$ for $\tilde{a}_{\kappa}$, viewed as a map $\mathbb{S}^{3} \rightarrow S U(2) \simeq \mathbb{S}^{3}$, is equal to $c_{2}=\kappa$ (with the appropriate choices of the orientations). Correspondingly, $O_{\kappa(\infty)}=\varphi \circ \tilde{O}_{(\kappa(\infty)}$ is a gauge at infinity for $a_{\kappa}$, and since $\varphi$ induces the isomorphism $\pi_{3}(S U(2)) \rightarrow \pi_{3}(\mathbf{G})$, we have $\left[O_{\kappa(\infty)}\right]=-\kappa[\varphi]$. Since $\boldsymbol{\chi}$ is dependent only on the topological class, the claim follows.

Next, analogous to Theorem 1.30, we claim that:

Claim 6.3. Let a be a finite energy harmonic Yang-Mills connection, which is not an instanton. Then

$$
\mathcal{E}_{e}(a) \geq|\chi|+16 \pi^{2}
$$

In essence, this is [10, Corollary 1.2]. However, to insure that we obtain the sharp bound, we need to verify that the proof goes through for our choice of $\langle\cdot, \cdot\rangle$, without relying on an embedding $\mathfrak{g} \subset s o(n)$ to normalize $\langle\cdot, \cdot\rangle$ as in [10]. For this purpose, we have the following replacement of [10, Lemma 2.1]:

Lemma 6.4. Under our normalization (6.3), we have

$$
|[A, B]| \leq \sqrt{2}|A||B| \quad \text { for any } A, B \in \mathfrak{g} .
$$

with equality if and only if, up to an $A d(O)$-action, $A$ and $B$ are proportional to two of $\left\{\mathbf{i}_{\alpha}, \mathbf{j}_{\alpha}, \mathbf{k}_{\alpha}\right\}$ for some highest root $\alpha$.

Proof. Consider a maximal abelian subalgebra $\mathfrak{h}$ containing $A$. Eigenvalues of $\operatorname{ad}(A)$ are

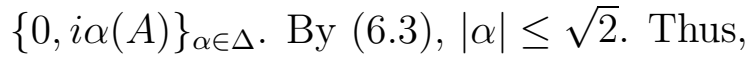

$$
|[A, B]|=|a d(A) B| \leq \sup _{\alpha \in \Delta}|\alpha(A)||B| \leq \sup _{\alpha \in \Delta}|\alpha||A||B| \leq \sqrt{2}|A||B| .
$$

In order for the equalities to hold, $\alpha$ must be a highest root, $A=|A| H_{\alpha}=|A| \mathbf{k}_{\alpha}$, and $B \in \operatorname{span}\left(\mathbf{i}_{\alpha}, \mathbf{j}_{\alpha}\right)$. Since $A d\left(\exp \left(s \mathbf{k}_{\alpha}\right)\right)$ simply rotates the plane $\operatorname{span}\left(\mathbf{i}_{\alpha}, \mathbf{j}_{\alpha}\right)$, and leaves $\mathbf{k}_{\alpha}$ invariant, we see that $A d\left(\exp \left(s \mathbf{k}_{\alpha}\right)\right) B$ is parallel to $\mathbf{i}_{\alpha}$ for an appropriate choice of $s \in \mathbb{R}$. Finally, the converse is easy to verify.

The proof in [10] now goes through for a G-bundle with normalization (6.3) with the parameters $\gamma_{0}=\sqrt{2}$ and $\gamma_{1}=\frac{2}{\sqrt{3}} \gamma_{0}=\frac{4}{\sqrt{6}}$. The $S U(2)$-instanton with $\kappa=1$, which we constructed above, saturates the inequalities in [10], exactly as in [10, Remark 2.7 and Section 3.2].

Step 2: $\mathrm{G}$ is a general nonabelian compact Lie group. Finally, we consider a general nonabelian compact Lie group $\mathbf{G}$ and prove Theorem 1.32 .

Observe that the $A d$-invariant inner product on $\mathfrak{g}$ can be used to define the orthogonal complement $\mathfrak{h}^{\perp}$ of an ideal $\mathfrak{h} \subseteq \mathfrak{g}$, which is also an ideal. Thus $\mathfrak{g}$ admits the direct-sum splitting

$$
\mathfrak{g}=\tilde{\mathfrak{g}}_{1} \oplus \cdots \oplus \tilde{\mathfrak{g}}_{\tilde{n}}
$$


as Lie algebra ideals, where each summand has no proper nonzero ideal. In fact, it is either 1-dimensional, and thus abelian, or simple. Since $\mathbf{G}$ is assumed to be nonabelian, at least one summand is simple. Thus, we arrive at the decomposition

$$
\mathfrak{g}=\mathfrak{g}_{1} \oplus \cdots \oplus \mathfrak{g}_{n} \oplus \mathfrak{a} .
$$

where $n \geq 1$, each $\mathfrak{g}_{i}$ is simple, and $\mathfrak{a}$ is abelian. As a result, the universal cover $\tilde{\mathbf{G}}$ of $\mathbf{G}$ splits into

$$
\tilde{\mathbf{G}}=\Pi_{i} \mathbf{G}_{i} \times \mathbb{R}^{r}
$$

where $\mathbf{G}_{i}$ is the simply connected Lie group corresponding to $\mathfrak{g}_{i}$, and $r=\operatorname{dim} \mathfrak{a}$. Denote by $\boldsymbol{\pi}_{i}$ the projection $\mathbf{G} \rightarrow \mathbf{G}_{i}$, and by $\mathrm{d} \boldsymbol{\pi}_{i}$ the corresponding projection $\mathfrak{g} \rightarrow \mathfrak{g}_{i}$, with the convention $\mathbf{G}_{n+1}=\mathbb{R}^{r}, \mathfrak{g}_{n+1}=\mathfrak{a}$.

As we are working with global gauge potentials on $\mathbb{R}^{4}$, the splitting allows us to decompose any $a$ into components $\mathrm{d} \boldsymbol{\pi}_{i}(a)$, which are completely decoupled from each other. We have the splitting

$$
\begin{aligned}
\boldsymbol{\chi} & =\int_{\mathbb{R}^{4}}-\langle F[a], F[a]\rangle=\sum_{i} \int_{\mathbb{R}^{4}}-\langle\mathrm{d} \boldsymbol{\pi}(F[a]), \mathrm{d} \boldsymbol{\pi}(F[a])\rangle=\sum_{i} \boldsymbol{\chi}\left(\mathrm{d} \boldsymbol{\pi}_{i}(a)\right), \\
\mathcal{E}_{e}(a) & =\frac{1}{2} \int_{\mathbb{R}^{4}}\left\langle F_{j k}[a], F^{j k}[a]\right\rangle=\sum_{i} \frac{1}{2} \int_{\mathbb{R}^{4}}\left\langle\mathrm{~d} \boldsymbol{\pi}\left(F_{j k}[a]\right), \mathrm{d} \boldsymbol{\pi}\left(F^{j k}[a]\right)\right\rangle=\sum_{i} \mathcal{E}_{e}\left(\mathrm{~d} \boldsymbol{\pi}_{i}(a)\right) .
\end{aligned}
$$

Moreover, $a$ is a harmonic Yang-Mills connection if and only if each $\mathrm{d} \boldsymbol{\pi}_{i}(a)$ is. In this case, $\mathrm{d} \boldsymbol{\pi}_{n+1}(a)=0$, since no nontrivial finite energy harmonic 2 -form exists on $\mathbb{R}^{4}$.

For each compact simple $\mathbf{G}_{i}$, let $E_{i}$ be the energy of a first instanton; from Step 1, we know that $E_{i}=\frac{16}{\langle\alpha, \alpha\rangle} \pi^{2}$, where $\alpha$ is a highest root in $\mathfrak{g}_{i}$. Reordering the factors if necessary, we may arrange so that $E_{1} \leq E_{2} \leq \ldots \leq E_{n}$. In particular, $E_{1}$ coincides with the infimum in Theorem 1.32, and part (1) follows.

To prove part (2), note that if $a$ is a finite energy harmonic Yang-Mills connection with energy $<2 E_{1} \leq 2 E_{i}$, then by Step 1 , each $\mathrm{d} \boldsymbol{\pi}_{i}(a)$ is either zero or a first instanton. Immediately by (6.5), we also see that exactly one of $\mathrm{d} \boldsymbol{\pi}_{i}(a)$ is nonzero. Thus $|\boldsymbol{\chi}|=\left|\boldsymbol{\chi}\left(\mathrm{d} \boldsymbol{\pi}_{i}(a)\right)\right|=\mathcal{E}_{e}\left(\mathrm{~d} \boldsymbol{\pi}_{i}(a)\right)=\mathcal{E}_{e}(a)$, as desired.

\section{REFERENCES}

[1] M. F. Atiyah, N. J. Hitchin, V. G. Drinfeld, and Y. I. Manin, Construction of instantons, Phys. Lett. A 65 (1978), no. 3, 185-187, doi.

[2] M. F. Atiyah, N. J. Hitchin, and I. M. Singer, Self-duality in four-dimensional Riemannian geometry, Proc. Roy. Soc. London Ser. A 362 (1978), no. 1711, 425-461, doi.

[3] G. Bor, Yang-Mills fields which are not self-dual, Comm. Math. Phys. 145 (1992), no. 2, 393-410, 1ink.

[4] R. Bott, An application of the Morse theory to the topology of Lie-groups, Bull. Soc. Math. France 84 (1956), 251-281, link.

[5] P. T. Chruściel and E. Delay, On mapping properties of the general relativistic constraints operator in weighted function spaces, with applications, Mém. Soc. Math. Fr. (N.S.) (2003), no. 94, vi+103.

[6] J. Corvino, Scalar curvature deformation and a gluing construction for the Einstein constraint equations, Comm. Math. Phys. 214 (2000), no. 1, 137-189, doi.

[7] J. Corvino and R. M. Schoen, On the asymptotics for the vacuum Einstein constraint equations, J. Differential Geom. 73 (2006), no. 2, 185-217, link.

[8] S. Czimek, An extension procedure for the constraint equations, preprint (2016), arXiv:1609.08814.

[9] _ Boundary harmonic coordinates and the localised bounded $L^{2}$ curvature theorem, preprint (2017), arXiv: 1708.01667 
[10] M. Gursky, C. Kelleher, and J. Streets, A conformally invariant gap theorem in Yang-Mills theory, preprint (2017), arXiv:1708.01157.

[11] S. Klainerman and M. Machedon, Finite energy solutions of the Yang-Mills equations in $\mathbb{R}^{3+1}$, Ann. of Math. (2) 142 (1995), no. 1, 39-119, doi.

[12] A. W. Knapp, Lie groups beyond an introduction, 2nd ed., Progress in mathematics, vol. 140, Birkhäuser, 2002.

[13] S. Kobayashi and K. Nomizu, Foundations of differential geometry. Vol. I, Wiley Classics Library, John Wiley \& Sons, Inc., New York, 1996, Reprint of the 1963 original, A Wiley-Interscience Publication.

[14] _ Foundations of differential geometry. Vol. II, Wiley Classics Library, John Wiley \& Sons, Inc., New York, 1996, Reprint of the 1969 original, A Wiley-Interscience Publication.

[15] J. Krieger and D. Tataru, Global well-posedness for the Yang-Mills equation in $4+1$ dimensions. Small energy, Ann. of Math. (2) 185 (2017), no. 3, 831-893.

[16] J. W. Milnor and J. D. Stasheff, Characteristic classes, Princeton University Press, Princeton, N. J.; University of Tokyo Press, Tokyo, 1974, Annals of Mathematics Studies, No. 76.

[17] S.-J. Oh, Gauge choice for the Yang-Mills equations using the Yang-Mills heat flow and local wellposedness in $H^{1}$, J. Hyperbolic Differ. Equ. 11 (2014), no. 1, 1-108, doi.

[18] Finite energy global well-posedness of the Yang-Mills equations on $\mathbb{R}^{1+3}$ : an approach using the Yang-Mills heat flow, Duke Math. J. 164 (2015), no. 9, 1669-1732, doi.

[19] S.-J. Oh and D. Tataru, Local well-posedness of the $(4+1)$-dimensional Maxwell-Klein-Gordon equation at energy regularity, Ann. PDE 2 (2016), no. 1, Art. 2, 70, arXiv:1503.01560, doi.

[20] , The Yang-Mills heat flow and the caloric gauge, preprint (2017), arXiv:1709.08599.

[21] The hyperbolic Yang-Mills equation in the caloric gauge. Local well-posedness and control of energy dispersed solutions, preprint (2017), arXiv:1709.09332.

[22] _ The Threshold Conjecture for the energy critical hyperbolic Yang-Mills equation, preprint (2017), arXiv:1709.08606.

[23] _ The Threshold Theorem for the $(4+1)$-dimensional Yang-Mills equation: An overview of the proof, preprint (2017), arXiv:1709.09088.

[24] T. H. Parker, A Morse theory for equivariant Yang-Mills, Duke Math. J. 66 (1992), no. 2, 337-356, doi.

[25] L. Sadun and J. Segert, Non-self-dual Yang-Mills connections with nonzero Chern number, Bull. Amer. Math. Soc. (N.S.) 24 (1991), no. 1, 163-170, doi.

[26] R. Schoen and K. Uhlenbeck, Boundary regularity and the Dirichlet problem for harmonic maps, J. Differential Geom. 18 (1983), no. 2, 253-268, link.

[27] L. M. Sibner, R. J. Sibner, and K. Uhlenbeck, Solutions to Yang-Mills equations that are not self-dual, Proc. Nat. Acad. Sci. U.S.A. 86 (1989), no. 22, 8610-8613, doi.

[28] E. M. Stein, Singular integrals and differentiability properties of functions, Princeton Mathematical Series, No. 30, Princeton University Press, Princeton, N.J., 1970.

[29] T. Tao, Local well-posedness of the Yang-Mills equation in the temporal gauge below the energy norm, Journal of Differential Equations 189 (2003), no. 2, 366 - 382, doi.

[30] K. K. Uhlenbeck, Connections with $L^{p}$ bounds on curvature, Comm. Math. Phys. 83 (1982), no. 1, $31-42$, link.

[31] _ The Chern classes of Sobolev connections, Comm. Math. Phys. 101 (1985), no. 4, 449-457, link.

Department of Mathematics, UC Berkeley, Berkeley, CA 94720 and Kias, Seoul, Korea 02455

Email address: sjoh@math.berkeley.edu

Department of Mathematics, UC Berkeley, Berkeley, CA 94720

Email address: tataru@math. berkeley.edu 